\title{
Eastern red-backed salamanders: A comprehensive review of an undervalued model in evolution, ecology, \& behavior
}

\author{
M. Caitlin Fisher-Reid ${ }^{1}$, Kristine Grayson ${ }^{1}$, Sara R. Grouleff ${ }^{1}$, Madelyn A. Hair ${ }^{1}$, Tanya J. \\ Hawley Matlaga ${ }^{1}$, Angelina K. Ireland ${ }^{1}$, Louise S. Mead ${ }^{1}$, Alyssa St John ${ }^{1}$, Meigan Starr ${ }^{1}$, \\ Sean C. Sterrett ${ }^{1}$, and Kody N. Streeter ${ }^{1}$ \\ ${ }^{1}$ Affiliation not available
}

August 3, 2021

\begin{abstract}
What makes a model organism? Identifying the qualities of a model organism has been given a great deal of attention in the biomolecular sciences, but less so in the fields of evolution, ecology, and behavior (EEB). In EEB, biotic and abiotic variation are features to understand, not bugs to get rid of, and EEB scientists often select organisms to study which best suit the scientific question at hand. Successful EEB model organisms can be studied at multiple biological scales and have a wealth of accumulated knowledge on which current research programs build. A recent call within EEB to invest in the inclusive development of diverse model systems and scientists has led us to evaluate the standing of the widespread, abundant, terrestrial salamander we study, the eastern red-backed salamander (Plethodon cinereus). We first look at salamanders as EEB models more generally, to determine where $P$. cinereus fits in this broader context. We next present a comprehensive review of the literature on the eastern red-backed salamander (Plethodon cinereus) since the last comprehensive review was completed in 1998. The core of our paper reviews 410 recent studies and highlights inconsistencies, gaps in our knowledge, and future directions in the context of the 1998 review. Finally, we present a collaborative research network, SPARCnet, as a nascent infrastructure for continued research on $P$. cinereus. Here, we especially discuss how this type of infrastructure can be broadly applied not just to other salamanders, but to other model systems, so that the future of EEB research may benefit from models which accurately represent, in Darwin's words, "endless forms most beautiful and most wonderful."
\end{abstract}

\section{Authors}

M. Caitlin Fisher-Reid ${ }^{1 *}$, Kristine L. Grayson ${ }^{2}$, Sara R. Grouleff ${ }^{3}$, Madelyn A. Hair ${ }^{2}$, Tanya J. Hawley Matlaga $^{4}$, Angelina K. Ireland ${ }^{3}$, Louise S. Mead ${ }^{5}$, Alyssa St John ${ }^{1}$, Meigan Starr ${ }^{5}$, Sean C. Sterrett ${ }^{3}$, and Kody N. Streeter ${ }^{4}$

\section{Affiliations}

${ }^{1}$ Bridgewater State University, Bridgewater, MA 02325; *mfisherreid@bridgew.edu; 508-531-1859; ${ }^{2}$ University of Richmond, Richmond, VA 23173; ${ }^{3}$ Monmouth University, West Long Branch, NJ 07764; ${ }^{4}$ Susquehanna University, Selinsgrove, PA $17870 ;{ }^{5}$ Michigan State University, East Lansing, MI 48824

Key words: model organism; Plethodontidae; Plethodon cinereus; amphibians; ecology; evolution; behavior 


\section{Contents}

I. Introduction

II. Model Systems in Evolution, Ecology, and Behavior

III. Salamanders \& the Plethodontidae as Model Organisms in EEB

IV. Methods

(1) Literature Searches

(2) Estimation of Geographic Range Size

(3) Comprehensive Literature Review of $P$. cinereus

V. Results

(1) Literature Searches

(2) Estimation of Geographic Range Size

(3) Comprehensive Literature Review of $P$. cinereus

(a) Identification

(b) Systematics and Geographic Variation

(c) Distribution and Adult Habitat

(d) Breeding and Courtship

(e) Reproductive Strategy

(f) Terrestrial Ecology

(i) Diet and Feeding Behavior

(ii) Relative Abundance and Density

(iii) Seasonal Activity and Migrations

(iv) Territories and Home Range Size

(v) Miscellaneous Behaviors and Sampling Methods

(g) Predators and Defense

(i) Predators and Parasites

(ii) Differential Predation Risk by Color

(iii) Defensive Behaviors

(h) Community Ecology

(i) P. shenandoah and P. cinereus Interactions

(ii) Other Salamanders and P. cinereus Interactions

(iii) Non-salamander Species and $P$. cinereus Interactions

(i) Conservation Biology

(j) Additional Topics

(i) Ecosystem Ecology 
(ii) Management

(iii) Microbiota and Disease

(iv) Ecotoxicology

VI. Is Plethodon cinereus an EEB Model Organism?

(1) Go big or go home: Long-term studies over large spatial scales

(2) A Nascent Infrastructure for Plethodon salamanders in SPARCnet: The Salamander Population and Adaptation Research Collaboration Network

VII. Conclusions

VIII. Acknowledgements

IX. Author Contributions

X. Literature Cited

XI. Supporting Information

\section{Introduction}

What defines a model organism for a particular biological field? While this idea has captured much discussion in the biomolecular literature (e.g., Müller \& Grossniklaus, 2010; Ankeny \& Leonelli, 2011; Leonelli \& Ankeny, 2013; Russell et al., 2017), the fields of evolution, ecology, and behavior (hereafter, EEB) have historically focused less on centering their science around specific study systems and more on studying the best system suited to answer the scientific question at hand. Yet, even without a broader community discussion, particular research systems in EEB stand out for their body of knowledge and contributions to unifying principles and researchers often invoke their study species as being excellent models for "robust inference" (sensu Travis, 2006). At the Virtual Asilomar American Society of Naturalists Vice Presidential Symposium in January 2021, Duffy et al. (2021) laid out a broad and inclusive framework for defining, developing, and nurturing model organisms and model systems in EEB, including describing important contrasts with the biomolecular sciences. Similarly, in a President's Plenary talk for the Society for Systematic Biologists in June 2021, Erika Edwards discussed the value of a model clade to EEB and systematic biology in particular. In this paper, we argue that one of the most common vertebrates in northeastern North American forests, the eastern redbacked salamander, (Plethodon cinereus), deserves recognition and further development as an EEB model organism.

We make this argument based on several factors, including (1) the great depth of knowledge already published on this species that highlights the tractability and potential of $P$. cinereus both in the laboratory and field for answering EEB questions (Petranka, 1998; Jaeger et al., 2016; studies reviewed herein), (2) the critical niche position of woodland salamanders in forest ecosystems (Davic \& Welsh, 2004), (3) the accessibility of this species as easy to find, human-tolerant, and locally abundant across a large geographic and climatic range (Fig. 1; Petranka, 1998) and (4) the potential for transferable methods and knowledge to such a wide range of salamanders given the extensive diversity, abundance (Plethodontidae comprise nearly $2 / 3$ of all salamander species), and well-studied systematics of the family. In making our argument, we review current knowledge of this species to build a resource for scientists looking to work with $P$. cinereus and discuss the next steps in investment and infrastructure needed for model organisms like $P$. cinereus to be successful (Ankeny \& Leonelli, 2011; Leonelli \& Ankeny, 2013; Duffy et al., 2021; Wale \& Duffy, 2021).

To that end, our paper has four overarching goals. First, we briefly discuss the definition and use of model organisms in EEB, highlighting salamanders as good candidates for model organism status. Second, we 
determine the current level of EEB knowledge on P. cinereus compared to other well-studied salamander species. Third, we share the results of a comprehensive literature review of the EEB research on $P$. cinereus that has occurred since the last comprehensive review, more than 20 years ago by Petranka (1998). Finally, we discuss the challenges of advancing $P$. cinereus and similarly well-studied species into the next stage of model organism status. Here, we highlight a collaborative research network, SPARCnet (the Salamander Population and Adaptation Research Collaboration Network), which offers a nascent infrastructure necessary for continued development of $P$. cinereus as a model system in EEB.

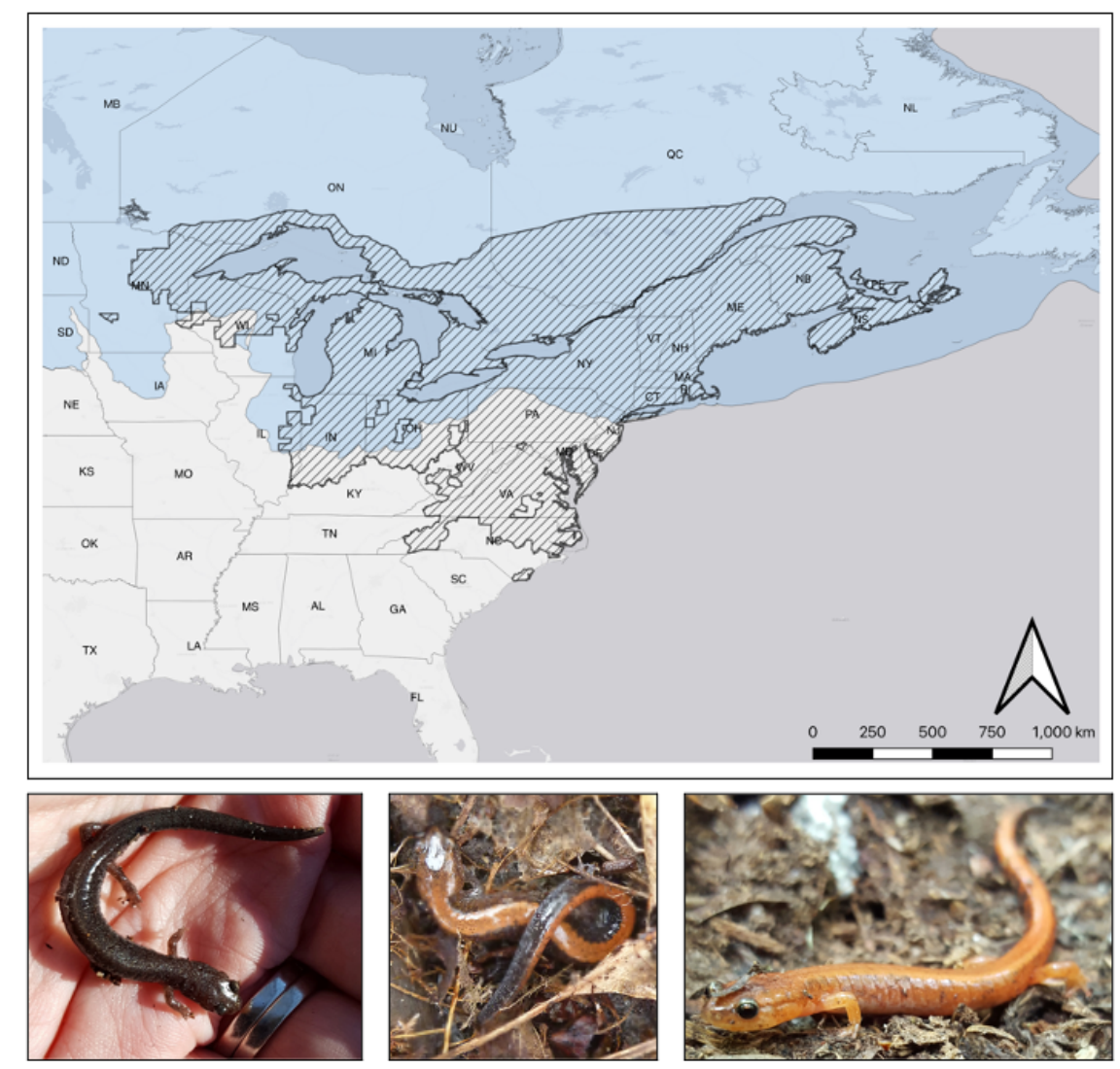

Figure 1. Top: Geographic range of Plethodon cinereus shown as diagonal lines is 1.89 million $\mathrm{km}^{2}$ (IUCN, 2020). The blue shaded area represents the extent of the Wisconsin ice sheet at the last glacial maximum (CRC, 2014). Bottom: The three most common color phenotypes of $P$. cinereus, from left to right, unstriped, striped, and erythristic (Photos by M.C. Fisher-Reid).

\section{Model Systems in Evolution, Ecology, and Behavior}

As part of defining what model organisms are for EEB, Duffy et al. (2021) summarize the considerations for using model organisms in cellular, molecular, and developmental biology (hereafter collectively referred to as the biomolecular sciences; e.g., Müller \& Grossniklaus, 2010; Ankeny \& Leonelli, 2011; Leonelli \& Ankeny, 2013; Russell et al., 2017), aptly noting that these fields tend to neglect, or even negate, the ecology and evolution of their models. For example, Müller \& Grossniklaus (2010) highlight the origins of 19 model organisms and their major contributions to biology. However, the contributions of these 19 taxa to the fields 
of EEB are largely overlooked, despite the prominent phylogeny the authors include and implicit nod at EEB when highlighting the discovery of the highly conserved HOX genes in Drosophila. Similarly, Russell et al. (2017) profile 14 "non-model model organisms" and focus on fundamental phenomena at the scale of molecules, genes, and cells with little regard for EEB fundamental phenomena (as defined by Ankeny \& Leonelli, 2011; Leonelli \& Ankeny, 2013). There is some discussion of how non-model model organisms have been used to study major evolutionary events (e.g., the origins of multicellularity with Volvox; Russell et al., 2017), however, the fields of EEB are not well-represented in discussions of model organisms as a whole. While both reviews focus on the biomolecular utility of model organisms, the EEB scientific community values many of these same organisms (e.g., Escherichia coli, D. melanogaster, Arabidopsis thaliana; Duffy et al., 2021), often for many of the same reasons (e.g., short generation time, lab tractability). Despite these overlaps in utility for both fields, however, Duffy et al. (2021) argue that there are a number of challenges in applying the definition of model organism from the biomolecular sciences to EEB studies. Instead, Duffy et al. (2021) propose the following definition for a model system in EEB (p. 55):

"a species, taxon, community, or ecosystem that has been studied from multiple angles with a goal of developing a deep understanding of that organism (or taxon, community, or ecosystem), in a manner that enables comparisons with other systems to illuminate general ecological, evolutionary, and/or behavioral principles; achieving this will require that the system has been studied long enough for a substantive body of knowledge to have been generated."

In thinking about qualities that model systems in EEB share, the authors identify three axes which define the use of a given model system for a particular research question: lab tractability, field tractability, and the range of biological scales which can be addressed (see Figure 1 in Duffy et al., 2021). It is important to highlight that their approach defines model systems - not always just a single species - which more accurately reflects how EEB scientists generally approach science from the perspective of larger biological scales compared to the biomolecular sciences. Individual organisms are often the smallest unit of interest in EEB, and although the burgeoning fields of molecular ecology, evolutionary genetics, and evolutionary development can focus on molecular mechanisms, the driving questions, even in these more molecular EEB fields, often focus on using molecular tools for understanding the whole organism or higher biological scales. We add to Duffy et al. (2021) that model systems in EEB often benefit from encompassing taxa that are locally abundant and/or span relatively large geographic ranges, as these factors contribute to both the accessibility and potential for lessons learned studying the model system to have broader applicability.

The exemplary taxa in EEB textbooks and research programs often take the form of model systems: groups of closely related, ecologically diverse species, found in relatively confined geographic regions (e.g., Anolis lizards, Darwin's finches, Heliconius butterflies), which lend themselves to addressing a variety of broader EEB research questions. Instead of using common ancestry and evolutionarily conserved traits to declare our model systems representative of other species, as is done in the biomolecular sciences, EEB tries to conceptually unify the natural world. As Duffy et al. (2021) state, EEB scientists "aim to see the forest and the trees" (their emphasis; p. 53).

One of the most crucial challenges facing the use of biomolecular model organisms for EEB research is that these lab-evolved populations are often dramatically different from their wild relatives in both ecologically and evolutionarily important ways (Duffy et al., 2021). These model organisms have long been artificially selected by scientists to produce standardized laboratory strains amenable to mass rearing, such that they are often deliberately stripped of both genotypic and phenotypic variation, and the ecological context for this variation. Thus, biomolecular model organisms generally violate one of the primary requirements for a model organism to address EEB-related questions: that an organism or model system be representative of other species and inform our understanding of general principles or fundamental phenomena in the natural world (Wale \& Duffy, 2021).

We, therefore, explicitly define the fundamental phenomena of EEB in the context of considering if a species or system can serve as a model organism in EEB (Fig. 2). In evolution, research focuses largely on the survival and reproduction of organisms based on their hereditary phenotypic variation and seeks to explain 
how traits change over time in the context of common ancestry. In ecology, research focuses on the spatial and temporal distribution and abundance of organisms, and how their interactions with each other and their abiotic environments drive population and community dynamics (Begon, Townsend, \& Harper, 2006). Behavior unites ecology and evolution with the organismal sciences of physiology, genetics, and development to understand the how and why of behavioral traits (i.e., Tinbergen's four questions; (Tinbergen, 1963). Historically thought to be restricted to animals, we now know that evidence of behavior can be found in all life forms, from microbes that cooperate when resources are limited (e.g., Dictyostelium discoideum; Devreotes, 1989), to plants that use chemical cues to warn neighbors of impending herbivore attacks (Babikova et al., 2013). We propose that all research questions in EEB can be assigned to one or more of these three broad contexts at their core, thus defining the fundamental phenomena of EEB (Fig. 2).

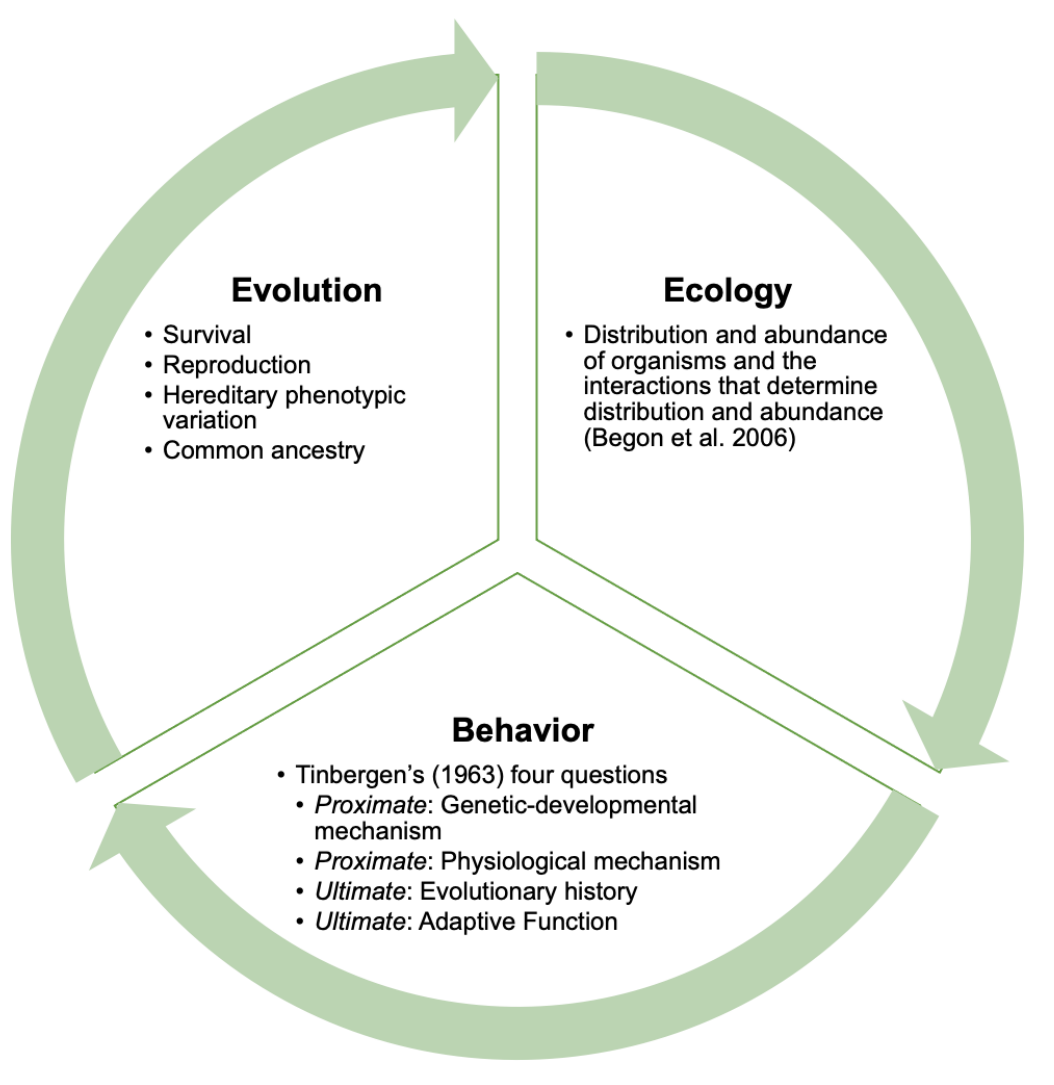

Figure 2. Fundamental phenomena in evolution, ecology, and behavior.

Important to the goals of this manuscript, and rightfully included by Duffy et al. (2021), model systems in EEB must contribute to robust inference across these fundamental phenomena (Travis, 2006). Both Travis (2006) and Duffy et al. (2021) argue that an organism or system must generate a great body of knowledge to support continued study of EEB fundamental phenomena. We demonstrate that $P$. cinereus has well surpassed the bar of knowledge, but requires further development of infrastructure, community ethos, and funding investment to facilitate continued robust inference on communities of woodland salamanders. 


\section{Salamanders \& the Plethodontidae as Model Organisms in EEB:}

Amphibians are represented in biomolecular model species by the axolotl (Ambystoma mexicanum; Russell et al., 2017) and the African clawed frog (Xenopus laevis; Müller \& Grossniklaus, 2010). In EEB, pondbreeding amphibians have long played a major role in studies of metamorphosis and complex life cycles (Wilbur, 1980). Despite pond-breeding salamanders being more commonly recognized, the salamander family Plethodontidae represents nearly two-thirds of all salamander species (486 of 750; AmphibiaWeb, 2020), and its members occupy a wide range of aquatic and terrestrial microhabitats, often with distinctly different life histories from pond-breeding amphibians (Wake \& Lynch, 1976; Petranka, 1998; Blankers, Adams, \& Wiens, 2012). Plethodontids are the only salamander family to have radiated extensively in the tropics (tropical bolitoglossines; Wake \& Lynch, 1976; Wiens, 2007), and the only family of salamanders with species that have direct development (i.e., no aquatic larval stage; Wake \& Hanken, 2004). In fact, because Plethodontidae is the largest salamander family, direct development is actually the most common mode of reproduction in salamanders and was likely a key innovation involved in the diversification of Plethodontidae (Schluter, 2000; Wake \& Hanken, 2004). Compared to pond-breeding amphibians, species of plethodontids with direct development generally have high field tractability and site fidelity, while pond-breeders can often only be sampled in large numbers during the brief breeding season. In terms of conservation status, plethodontid species run the gamut from least concern to endangered or data deficient (Table 1; IUCN, 2020).

Table 1. Conservation status for 408 species of Plethodontidae ( $54 \%$ of family) which have been evaluated by the IUCN (IUCN, 2020).

\begin{tabular}{ll}
\hline Conservation Status & Number of Plethodontidae Species \\
Least Concern & 94 \\
Near Threatened & 35 \\
Vulnerable & 65 \\
Endangered & 94 \\
Critically Endangered & 85 \\
Extinct & 2 \\
Data Deficient & 33 \\
\hline
\end{tabular}

As amphibians and ectotherms, salamanders are generally sensitive to their environment (Duellman \& Trueb, 1986). Ecologically, salamanders are often mid-level consumers who play a critical role in nutrient cycling and other ecosystem processes throughout the aquatic and/or terrestrial ecosystems where they live (Davic \& Welsh, 2004). Plethodontid salamanders are particularly sensitive to climate as these species are all lungless: all respiration is cutaneous, which requires moist skin. Climates and seasons that are too hot, too cold, or too dry are inhospitable to plethodontids. In part due to this environmental sensitivity, several studies have already made the case for the use of plethodontid salamanders in biodiversity and ecosystem monitoring as indicator species (Welsh \& Ollivier, 1998; Welsh \& Droege, 2001; Best \& Welsh, 2014; Farallo \& Miles, 2016; Fleming et al., 2020), while others have argued caution when using amphibians as 'canaries in a coal mine' for anthropogenic change (Kerby et al., 2010).

The genus Plethodon includes 58 terrestrial woodland species found in North America, for which the vast majority of species (49 species) form a clade of eastern Plethodon, while the remaining 9 species form a clade of western Plethodon, the majority of which are found in the Pacific Northwest (Wiens, Engstrom, \& Chippindale, 2006; Fisher-Reid \& Wiens, 2015; AmphibiaWeb, 2020). Phylogenetically, P. cinereus is a member of the basal clade of the three eastern North American clades of Plethodon (Wiens et al., 2006; Fisher-Reid \& Wiens, 2015) and it is the sister species to the vulnerable P. shenandoah (IUCN, 2020), which, while locally abundant where found, is geographically restricted to three mountaintops in Virginia 
(Petranka, 1998; Sites et al., 2004). Plethodon cinereus is a wide-ranging species found in temperate forest habitats along the eastern seaboard of North America from southern Canada to North Carolina, and west to Minnesota in the north, and the Appalachians in the south (Fig. 1). Within its range, P. cinereus is often locally abundant, with surface density estimates ranging from $0.05-3.3$ salamanders $/ \mathrm{m}^{2}$ (Petranka, 1998; Semlitsch, O'Donnell, \& Thompson, 2014), and more recent estimates reporting surface densities that can range higher (e.g., Hernández-Pacheco et al., 2019 reported densities of $2.5-6.3$ salamanders $/ \mathrm{m}^{2}$ in Richmond, Virginia).

As the most common and most widely distributed species in the genus Plethodon, we argue that P. cinereus is an ideal candidate for an EEB model species and provide an updated review of knowledge on this salamander. There is a wealth of natural history, evolutionary, ecological, and behavioral research that has been done on $P$. cinereus over the decades, however the last comprehensive review was conducted over 20 years ago as part of a book detailing the natural history of North American salamanders (Petranka, 1998). In the early 2000s, some authors wrote additional broad overviews of amphibian biology which include species-specific knowledge (Lannoo, 2005; Wells, 2007), but these compendiums largely rely the details found in Petranka (1998), with minimal updates. Fifty years of behavioral research on $P$. cinereus was recently summarized in Jaeger et al. (2016) in which the authors highlight Petranka (1998) as the authoritative account for $P$. cinereus life history and ecology (pg. 3). In order to quantify the body of knowledge on $P$. cinereus for EEB fields in comparison to other well-studied salamanders we conducted several rigorous literature searches. From these searches, we used the resulting studies on $P$. cinereus to comprehensively review the post-Petranka (1998) literature.

\section{Methods}

\section{(1) Literature Searches}

As a first step for establishing a species of woodland salamander (P. cinereus) as a model organism in EEB, we investigated its prevalence in the literature relative to other well-studied salamanders. We conducted three sets of taxonomically progressive Web of Science topic searches using the Web of Science All Databases for All Years (1864-present). All searches were conducted on 18 June 2020. The goal of our search strategy was to restrict our focused efforts to the most well-studied salamanders, first by identifying the most well-studied families, then genera within those families, and finally species within those genera.

In the family-level search, we searched for each of the ten families of Caudata. Each family was searched to include informal and formal mentions, e.g., we used the topic search keywordTS=(plethodontid*) for Plethodontidae (Table 2). There was one family, Proteidae, which returned a large number of non-salamanderrelated articles. For example, our original search string TS=(proteid*) also returned articles on Proteides butterflies. We modified the search string for this family only, to restrict results to amphibians (see Table 2). We recorded the number of articles found in these searches, and retained the top three families for the second search (bolded in Table 2). These three families, (Plethodontidae, Salamandridae, and Ambystomatidae) had $3-8 \mathrm{x}$ more articles than the next highest family (Hynobiidae; Table 2), and represent the dominant Caudata families and study organisms globally. Together, these three families include 50 genera and represent $85 \%$ (640/750 named species; AmphibiaWeb, 2020) of salamander species, with Plethodontidae alone representing $65 \%$ (486/750 at the time of the literature search; AmphibiaWeb, 2020) of salamander species.

In the genus-level search, we searched for the name of each genus within three retained families based on lists of genera from AmphibiaWeb (2020). As before, we searched Web of Science All Databases for All Years. These were new searches (i.e., not subsets of the first search) in order to capture articles which do not explicitly list the family name in the title, abstract, or author-provided keywords. Thus, there are numbers in Table 3 for a given genus which are greater than the numbers returned in the family-level search in Table 2. For these searches, genus name was the only keyword used, e.g., TS=(Plethodon). Often, the large numbers 
for a given genus are driven by one or two very well-studied species, so our cutoff for a genus to move on to the species-level search was 883 , i.e., the number of articles returned for $P$. cinereus' s species-level search (see next paragraph). Thus, we retained the top ten genera for the species-level search (Table 3). Together, these ten genera represent 24\% (179/750 named species; AmphibiaWeb, 2020) of salamander species. Search results for all 50 genera are included in Table S1.

In the species-level search, we searched for each of the 179 species within the ten retained genera to determine which species are the most well-studied in EEB as measured by the total number of articles in these fields. As before, we searched the Web of Science All Databases for All Years, however, the species-level search strings included two restrictions to ensure we (1) captured only relevant articles which focused on that species and (2) minimized pseudoreplication. For example, to search for articles on P. cinereus, we used the search string: TS=("Plethodon cinereus") NOT KP=("Plethodon cinereus") AND DT=(ARTICLE). This restricted the topic search to title, abstract, and author-provided keywords, and eliminated irrelevant sources which are included in topic searches because the scientific name is found in the Keywords Plus (KP) field. $\mathrm{KP}$ is an algorithmically generated field based on the references of sources (Clarivate, 2021), and it can inflate results if an article on a closely related species regularly cites articles with the focal species in the article titles. Limiting the document type (DT) to articles reduces instances of pseudoreplication, as data sets, FigShare figures, and conference abstracts associated with a single published study are all counted as individual records if document type is not restricted. As it is standard practice to state scientific names at least once in published literature in EEB, we did not include common names in our search, nor did we include any subgenera listed on AmphibiaWeb (2020). Any species with $>800$ articles $(\mathrm{N}=8,4.5 \%$ of species searched) were retained (Table 4) and the full records of all articles were exported from Web Of Science as tab-delimited files to be further processed in Excel. For each of these eight species, we retained articles that were broadly in the fields of natural history, ecology, evolutionary biology, behavior, or conservation biology, and discarded articles that were not in these fields. We did this by sorting the Web of Science results by source (SO; journal title) and then examining the titles and, if necessary, the abstracts of articles in all records, paying special attention to records in both broad-scope journals (e.g., Nature, Science ), and journals that do not typically publish EEB-related studies (e.g., Developmental Biology, Journal of Cell Science). Macroevolutionary articles using salamanders to explore broad questions in vertebrate evolution were also discarded, as these did not focus on the microevolution of the focal species. All discarded articles deemed outside the intended scope of our review based on these criteria, as well as any duplicate records were moved to a second tab (Tables S3-S10), and not included in the final tallies for the species-level search (Table 4). This filtering step was particularly important for species that are used heavily in cell and developmental biology research. For example, the axolotl (Ambystoma mexicanum), is a model laboratory organism for developmental biology, limb regeneration, embryology, and several other applied biomolecular fields, and articles in these fields are not concerned with the axolotl's natural history, ecology, evolution, or behavior. In the supplement, we report the raw number of articles returned for all 179 species (Table S2), and include the lists of retained EEB sources vs. removed non-EEB sources for each of the eight focal species (Tables S3-S10).

\section{(2) Estimation of Geographic Range Size}

We expected geographic range size to be related to how well-studied an organism is in EEB due to accessibility: species with larger geographic ranges offer greater opportunities for more individual scientists to study their local population. In order to determine how wide-ranging $P$. cinereus is compared to other salamanders, we downloaded geographic range data from the IUCN Red List for all 607 salamander species for which data was available as a single shapefile (IUCN, 2020). Using QGIS 3.12, we estimated the area $\left(\mathrm{km}^{2}\right)$ of all polygons using the field calculator formula \$area, and summed polygons for each species using the Dissolve with Stats plugin. 


\section{(3) Comprehensive Literature Review of $P$. cinereus}

Finally, we conducted a comprehensive literature review of articles on $P$. cinereus that were published after Petranka (1998). We started with the 677 EEB articles that remained from our species-level Web of Science search (Table S8) and cross-referenced this list with sources cited by Petranka (1998) in his chapter on $P$. cinereus (pg. 335-346). Of these 677 articles, 361 articles were published after Petranka (1998) went to press, covering 1997-2020. To these, we added an additional 49 recent studies (spanning 2005-2020) which were not recovered by Web of Science, but which we knew of based on our own prior knowledge of the $P$. cinereus literature (Greenhalgh \& Peacock, 2005). This brought the total number of sources to be reviewed to 410 , spanning 1997 - June 2020. We next categorized these articles based on what biological scale or fundamental phenomena they focused on, using a combination of Petranka's (1998) section headers and the section headers for species accounts on AmphibiaWeb (2020) as our categories (Table S11). Articles were categorized into a primary and secondary category based on titles and abstracts. One criticism of the $P$. cinereus literature we were aware of going into this review was that most studies are geographically limited to a single population or geographic region within the geographic range. In order to quantify this, we recorded the source location of the salamanders used in each study (state or province) while we categorized. Studies that included salamanders from multiple states were counted for each state (e.g., Fisher-Reid et al., 2013 included field data from NY, CT, and NJ, and was counted once for each of these three states), bringing our total number of study locations to 458. From this number, we excluded one study from Tennessee (Fitzpatrick, Shook, \& Izally, 2009) because it was not actually conducted within the range of $P$. cinereus, but within the range of congener $P$. ventralis. Of the remaining 457 study locations, seven studies were considered range-wide (1.5\%), and two studies had unknown sources of salamanders $(0.4 \%)$, and were excluded from further analyses. Our final number of study locations was 448.

Once categorized, we divided the studies equally among all authors to be read and summarized. We kept our summaries to a few sentences recorded in a "Key Results" field in a shared spreadsheet (Table S11). From these single article summaries, we then drafted category summaries and aligned these with Petranka (1998) chapter headers, which comprise the Comprehensive Literature Review section below. In reviewing the post-Petranka (1998) literature on $P$. cinereus, we hope to first provide a much-needed update on the current state of knowledge on $P$. cinereus, one which thoroughly examines the geographic variation, gaps in our knowledge, and lays out future research directions. Second, we demonstrate how our research network, SPARCnet, is poised to embrace the geographic variation, collect standardized long-term demographic data, and run statistically robust field-based experiments.

\section{Results}

\section{(1) Literature Searches}

Results for the family-level and genus-level searches are shown in Table 2 and Table 3, respectively, and the full results for all 50 genera are in Table S1 and for all 179 species in Table S2. In the species-level search (Table 4), in terms of raw numbers of articles in EEB related fields compared to non-EEB related fields, $P$. cinereus comes out 5th, behind two salamandrids from Europe (Triturus cristatus and Salamandra salamandra) and two ambystomatids from North America (Ambystoma tigrinum and A. maculatum). Like $P$. cinereus, all four of these species have large ranges and are locally abundant (Lannoo, 2005; Wells, 2007; IUCN, 2020). Unlike P. cinereus, they are all pond-breeding species with an aquatic larval stage. Plethodon cinereus is the only representative of Plethodontidae among the top eight most well-studied salamander species, which is surprising, given that Plethodontidae is the largest family of salamanders (AmphibiaWeb, 2020), and the only salamander family with direct developing species (Wake \& Hanken, 2004). However, the vast majority of salamander reproductive and developmental biology has been studied in species with 
an aquatic larval stage (e.g., see non-EEB sources for A. mexicanum; Table S4).

Table 2. Family-level searches in Web of Science (WoS). All searches were conducted on 18 June 2020. Bolded taxa were retained for the genus level search.

\begin{tabular}{|c|c|c|c|}
\hline Salamander Family & Family Search String & $\begin{array}{l}\text { Number of articles } \\
\text { found (WoS All } \\
\text { Databases, 1864-2020) }\end{array}$ & $\begin{array}{l}\text { Number of Species } \\
\text { (AmphibiaWeb 2020) }\end{array}$ \\
\hline Ambystomatidae & $\mathrm{TS}=($ ambystomatid $*)$ & 4396 & 32 \\
\hline Amphiumidae & $\mathrm{TS}=\left(\right.$ amphiumid $\left.^{*}\right)$ & 514 & 3 \\
\hline Cryptobranchidae & $\mathrm{TS}=\left(\right.$ cryptobranchid $\left.^{*}\right)$ & 919 & 4 \\
\hline Dicamptodontidae & $\mathrm{TS}=\left(\right.$ dicamptodontid $\left.^{*}\right)$ & 306 & 4 \\
\hline Hynobiidae & $\mathrm{TS}=($ hynobiid $*)$ & 1656 & 82 \\
\hline Plethodontidae & $\mathbf{T S}=\left(\right.$ plethodontid $\left.^{*}\right)$ & 6385 & 486 \\
\hline Proteidae & $\begin{array}{l}\mathrm{TS}=\text { (proteid* AND }^{*} \\
\text { (amphibia* OR } \\
\text { caudat* OR } \\
\text { salamander* OR } \\
\text { urodel*)) }^{*}\end{array}$ & 1294 & 8 \\
\hline Rhyacotritonidae & $\mathrm{TS}=($ rhyacotritonid $*)$ & 14 & 4 \\
\hline Salamandridae & $\mathbf{T S}=\left(\right.$ salamandrid $\left.^{*}\right)$ & 13114 & 122 \\
\hline Sirenidae & $\mathrm{TS}=\left(\right.$ sirenid $\left.^{*}\right)$ & 456 & 5 \\
\hline
\end{tabular}

Table 3. Genus-level searches in Web of Science (WoS). The top 10 salamander genera with the highest number of articles are shown here. All of these were retained for the next stage of searching as they all contained more articles than the number of articles for $P$. cinereus 's species-level search (833), while the remaining 40 genera fell short of this value. Search results for all 50 genera are reported Table S1. All searches were conducted on 18 June 2020.

\begin{tabular}{|c|c|c|c|}
\hline Family & Genus Search String & Number of articles found (WoS All Databases, 1864-2020) & Numbe \\
\hline Salamandridae & $\mathrm{TS}=($ Tarich $a)$ & 1337 & 4 \\
\hline Plethodontidae & $\mathrm{TS}=($ Eurycea $)$ & 1463 & 33 \\
\hline Plethodontidae & $\mathrm{TS}=($ Desmognathus $)$ & 1700 & 22 \\
\hline Salamandridae & $\mathrm{TS}=($ Cynops $)$ & 1846 & 9 \\
\hline Salamandridae & $\mathrm{TS}=($ Pleurodeles $)$ & 2438 & 3 \\
\hline Salamandridae & $\mathrm{TS}=($ Notophthalmus $)$ & 2527 & 3 \\
\hline Plethodontidae & $\mathrm{TS}=($ Plethodon $)$ & 3040 & 58 \\
\hline Salamandridae & $\mathrm{TS}=($ Salamandra $)$ & 3054 & 6 \\
\hline Salamandridae & $\mathrm{TS}=($ Triturus $)$ & 9292 & 9 \\
\hline Ambystomatidae & $\mathrm{TS}=($ Ambystoma $)$ & 10937 & 32 \\
\hline
\end{tabular}

Table 4. Species-level searches in Web of Science. The top eight salamander species with the highest raw number of articles are shown here. The raw number includes all results for a given search string, while the adjacent column on the right includes only those articles directly related to the natural history, ecology, and evolution of the species (with duplicates removed). Raw number search results and search strings for all 179 species are reported in Table S2, and article lists for each of these eight species are reported in Tables S3-S10. All searches were conducted on 18 June 2020. 


\begin{tabular}{lllll}
\hline Geographic Region & Geographic Range Size $\mathbf{~ k m}^{2} ; \mathbf{I U C N ~ 2 0 2 0 )}$ & IUCN Threat Status & Family & S \\
Mexico & $1.03 \times 10^{2}$ & Critically Endangered & Ambystomatidae & \\
Europe & $4.37 \times 10^{6}$ & Least Concern & Salamandridae & T \\
North America & $5.15 \times 10^{6}$ & Least Concern & Ambystomatidae & \\
North America & $6.66 \times 10^{6}$ & Least Concern & Salamandridae & 1 \\
Europe & $2.16 \times 10^{6}$ & Least Concern & Salamandridae & $S$ \\
North America & $3.11 \times 10^{6}$ & Least Concern & Ambystomatidae & \\
Japan & $2.87 \times 10^{5}$ & Near Threatened & Salamandridae & $C$ \\
North America & $1.89 \times 10^{6}$ & Least Concern & Plethodontidae & $I$ \\
\hline
\end{tabular}

\section{(2) Estimation of Geographic Range Size}

Among all salamanders for which range data was available (N =607; IUCN, 2020), P. cinereus has the 11th largest range (Table S12). Among the top eight most well-studied salamander species (Table 4), five have geographic range sizes larger than $P$. cinereus, supporting the idea that well-studied species often have large ranges. Among plethodontids, $P$. cinereus has the second largest range overall (1.89 million $\mathrm{km}^{2}$; Table S12; IUCN, 2020), and the largest range of North American plethodontids. The tropical South American plethodontid, Bolitoglossa altamazonica is reported to have the largest plethodontid range at 1.97 million $\mathrm{km}^{2}$ (Table S12; IUCN, 2020). However, recent molecular and morphological analyses of B. altamazonica and B. peruviana (Cusi et al., 2020; Jaramillo et al., 2020) suggest B. altamazonica has previously been used as a "catchall" taxon for any specimens from Venezuela to Bolivia and from Ecuador to Brazil, and that instead B. altamazonica is a microendemic species restricted to forests in northern Peru, between the rivers Nanay in the north, Tigre and Marañón in the south, and Amazon in the west. Therefore, it is likely that $P$. cinereus has the largest range of plethodontids.

\section{(3) Comprehensive Literature Review of $P$. cinereus}

The following sections summarize the results of our comprehensive literature review of $P$. cinereus research since Petranka (1998) and published prior to our search date in June 2020. We use the same section headers as Petranka (1998), but we have added subheaders for larger topics. We follow Petranka's (1998) organization of topics closely. We have taken special care to note when more recent research (1) supports what is written in Petranka (1998), (2) fills in a gap in understanding which Petranka (1998) identified, or (3) contradicts what is written in Petranka (1998). In the supplement, we include our categorization and key results summaries of these sources which we used to organize this review (Table S11). We also include the full list of sources returned by Web of Science on P. cinereus (Table S8) and note which of these were cited by Petranka, (1998) for completeness (Table S13).

\section{(a) Identification}

Petranka (1998) describes the two predominant color phenotypes (striped morph or red-back and unstriped morph or lead-back) as well as the erythristic or all-red morph (Fig. 1). Since Petranka (1998), documentation of other color phenotype anomalies (e.g., leucistic) from throughout the geographic range have been published regularly, often in Herpetological Review's Natural History Notes section (e.g., Mendyk, Augustine, \& Montague, 2010; Sawyers \& Novick, 2011; Jongsma, 2012a, 2012b). Recently, Moore \& Ouellet (2014) reviewed color phenotypes in $P$. cinereus, summarizing eight diagnosable color phenotypes with photographs. Moore \& Ouellet (2014) provide a dichotomous key for identifying the color phenotypes and are maintaining an open-access database of photographs and provide an email address for photograph submissions. The authors also provide a table documenting each state or province in which different morphs have been 
found, noting that the unstriped morph is absent at range edge populations in Minnesota, Kentucky, and Tennessee. Moore \& Ouellet (2014) argue that the striped, unstriped, and erythristic morphs (Fig. 1) should be considered the primary color phenotypes of $P$. cinereus as they are the most abundant and occur most widely through the geographic range, while the remaining five color phenotypes (iridistic, albino, leucistic, amelanistic, melanistic) are somewhat rare anomalies with patchy observations throughout the range.

Additional work on color phenotypes since Petranka (1998) includes studies on the expression of different color phenotypes over time and over development. Over time, Kraemer, Kissner, \& Adams (2012) observed decreasing brightness of dorsal and lateral pigmentation and increasing ventral reflectance in both striped and erythristic morphs kept in a laboratory. The authors hypothesize that the changes in brightness were due to upregulation of dermal pigments (Kraemer et al., 2012), but note that little is known about the cellular mechanisms of dermal pigmentation in this species. Kraemer et al. (2012) also caution that their study suggests laboratory quantification of coloration may not be representative of similar measurements recorded in natural settings.

Over development, Evans, Urban, \& Jockusch (2020) found that temperature may play a role in the expression of the color phenotype. The authors reared eggs at different temperatures, and found a higher proportion of unstriped individuals hatch at higher temperatures. The authors note, however, that it is difficult to know if this is true phenotypic plasticity in color phenotypes (i.e., high temperatures induce expression of unstriped morph) or simply differential mortality based on temperature (i.e., eggs containing unstriped individuals are more likely to survive rearing at high temperatures compared to eggs containing striped individuals; Evans et al., 2020). Further work is needed to tease these apart.

SPARCnet research has revealed an additional interesting trait related to skin phenotypes; one that is possibly sexually dimorphic. Muñoz (2018) describes natural green fluorescence under a blacklight on the ventral side of tails for 45 salamanders in Pennsylvania. Muñoz (2018) observed ventral tail fluorescence during the autumn (September to November) 2014 season and notes that the trait occurred more frequently in males $(\mathrm{N}=33)$ than females $(\mathrm{N}=12)$. In Massachusetts, one of us documented the same ventral fluorescence in 78 individual salamanders during the autumn 2019 season (M.C.F.-R.; unpublished data), as well as in a handful of individuals in the autumn seasons of 2017 and 2018 (prior to intentionally recording these observations; Fig. S1). In 2019, we also observed a strong male bias in fluorescence ( $\mathrm{N}=71$ males; 3 females; 4 unknowns) and we observed that fluorescence persists for several weeks, as 11 individuals recaptured 2-4 times (mean: 2.4) during the same season exhibited fluorescence each time they were caught (sample dates are generally [?] 7 days apart). Munoz (2018) speculates that the fluorescence may aid in night vision, but acknowledges that our lack of knowledge on how the fluorescent compounds are generated and on the natural light conditions under which they fluoresce makes it difficult to hypothesize as to their ecological or evolutionary importance.

Petranka (1998) notes minimal sexually dimorphic traits in $P$. cinereus, with sexually active males showing swollen nasolabial grooves, hedonic glands, and a mental gland. Two additional studies have been published since which describe non-invasive methods for sexing adult salamanders. Quinn \& Graves (1999b) report on sexual dimorphism in the inter-nares distance (distance between nares and snout tip). They show that males had significantly larger inter-nares distances than females, for salamanders whose sex was confirmed by dissection. While this trait is extremely useful for sexing preserved specimens without dissection, it is more difficult to use in field or laboratory settings with live animals. Measuring inter-nares distance on a live animal requires that the animal be cooled on ice prior to measuring, thus this method may not be practical in all field situations (Quinn \& Graves, 1999b). Gillette \& Peterson (2001) describe a candling method for determining sex non-invasively, which is used commonly today in both laboratory and field settings (e.g., Cabe et al., 2007; Novarro et al., 2018). By shining a light through the dorsal side of the salamander, and looking at the ventral body wall for shadows of testes and vas deferens (if male) or eggs (if female), one can determine sex. Gravid females can also have their eggs counted by pressing gently on the body wall to make the individual eggs visible through the ventral skin. Like any method for determining sex, candling has trouble distinguishing between sub-adult females with no eggs and sub-adult males with undeveloped testes, 
however, in our experience, it works well on adult salamanders (i.e., greater than $35 \mathrm{~mm}$ snout-vent-length (SVL); Petranka, 1998).

\section{(b) Systematics and Geographic Variation}

Genetic and bioinformatics tools for understanding the systematics and geographic variation of $P$. cinereus were only just becoming inexpensive and commonplace in 1998. The studies which Petranka (1998) cites on this topic are even older and rely exclusively on allozyme data to distinguish species-level and populationlevel genetic variation (Highton \& Webster, 1976; Highton \& Larson, 1979). However, the early inferences made from these data have largely held up under the scrutiny of both Sanger and next-generation sequencing methods, combined with more robust phylogenetic methods (e.g., Wiens et al., 2006; Fisher-Reid \& Wiens, 2011; Radomski et al., 2020). Today, we are confident that $P$. cinereus is genetically distinct from both its sister species P. shenandoah (Highton, 1999, p. 199; Sites et al., 2004; Wiens et al., 2006; Highton et al., 2012) and the closely related southern red-backed salamander (P. serratus; Wiens et al., 2006; Highton et al., 2012). At the time of our literature search, there was no published evidence of hybridization between $P$. cinereus and $P$. shenandoah (Carpenter, Jung, \& Sites, 2001), or between $P$. cinereus and either P. sherando or $P$. serratus (Bayer et al., 2012). However, rare hybrids between $P$. cinereus and $P$. shenandoah have recently been documented (E.H.C. Grant, pers. comm. ). There is also evidence of extensive hybridization between $P$. cinereus and P. electromorphus in Ohio (Lehtinen et al., 2016). Phylogenetically, P. electromorphus belongs to the cinereus clade and last shared a common ancestor with $P$. cinereus roughly 10 million years ago (Wiens et al., 2006). The results of Lehtinen et al. (2016) suggest there may be more cryptic hybridization yet to be found between $P$. cinereus and sympatric congeners within the cinereus clade.

The systematics of plethodontid salamanders has been one of the most active areas of research, both prior to Petranka's (1998) chapter, and after it. Because they are the largest family of salamanders, and the only group of salamanders to radiate in the tropics (Wake \& Lynch, 1976; Wiens, 2007), there has been much interest using them to study evolutionary questions in a phylogenetic context. From a systematics perspective, $P$. cinereus appears to behave as a single evolutionary species (i.e., individuals from across the range form a single phylogenetic lineage (Wiens et al., 2006; Fisher-Reid \& Wiens, 2011). A recent study has identified six phylogeographic clades within $P$. cinereus, based on both mitochondrial and nuclear genes (Radomski et al., 2020). Waldron et al. (2019) found a clinal transition between two of these clades in Ohio, detectable in the mitochondrial cytochrome $b$ gene, but not in the 10 nuclear microsatellite markers used. Waldron et al. (2019) suggest two scenarios to explain the discordance between mitochondrial and nuclear data: first the cline could be an artifact of genetic drift or lineage merger and second, that there may yet be a nuclear cline that is outside their sampling transect. The authors note that a genomic study with wider sampling is needed to fully understand the discordance between mitochondrial and nuclear genomes in $P$. cinereus .

At the population level, Petranka (1998) notes that P. cinereus populations in formerly glaciated regions are less genetically variable than southern populations that were never glaciated. This observation supports a post-glaciation range expansion from southern refugia, and has been further supported by more recent studies using either microsatellites (e.g., Cameron, Anderson, \& Page, 2017) or a combination of mitochondrial and nuclear genes (Radomski et al., 2020). Molecular ecology studies using microsatellites have been an area of active research since Petranka (1998). Connors \& Cabe (2003) published the first set of microsatellite markers for $P$. cinereus from a well-studied Virginia population, which have been widely used by researchers to test hypotheses of detectable isolation-by-distance (Cabe et al., 2007), the strength of natural and humanmade barriers to dispersal (Marsh et al., 2007, 2008), impacts of habitat fragmentation on urban populations (Gibbs, 1998c; Noel et al., 2007; Noel \& Lapointe, 2010; Wilk, Donlon, \& Peterman, 2020), kin discrimination (Liebgold \& Cabe, 2008), influence of historical landscape change on population structure (Jordan, Morris, \& Gibson, 2008), and color-biased dispersal (Grant \& Liebgold, 2017). The results of these studies are discussed in detail in the appropriate section later in this review. Here, we only note that Cabe et al. (2007) 
found low-level but detectable population structure over $2 \mathrm{~km}$ of continuous habitat in Virginia, consistent with an isolation-by-distance model, and no evidence for sex-biased dispersal. Grant \& Liebgold (2017) also found no evidence for sex-biased dispersal, but did find evidence for color-biased dispersal. In this Maryland population, striped salamanders showed population genetic evidence of philopatry, while unstriped salamanders showed population genetic evidence of dispersal (Grant \& Liebgold, 2017). It remains to be seen if this pattern exists in other populations.

Additional microsatellite marker sets were developed from populations on Long Island, New York (FisherReid et al., 2013), and from populations in Virginia, Ohio, and Pennsylvania (Cameron et al., 2017). Combined, these three marker sets cover five of the six phylogeographic clades identified by (Radomski et al., 2020), all except the North Carolina clade at the southwestern range limit. Population-level comparisons between the phylogeographic clades have been hampered by low variation in the formerly-glaciated regions (e.g., Fisher-Reid et al., 2013; Radomski et al., 2020) and difficulty amplifying markers outside of the populations they were developed in (e.g., Cameron et al., 2017). In completing this review, we expected that many of the studies on $P$. cinereus are done on a small subset of populations. This is, in fact, the case, with just over half of the reviewed studies occurring in or using salamanders from Virginia (29.5\% of studies reviewed), Ohio ( $13.2 \%$ of studies reviewed) or Pennsylvania ( $9.6 \%$ of studies reviewed), and indeed a large number of the population genetic studies mentioned here have limited geographic coverage (see additional comments below; Fig. 3; Table S14).

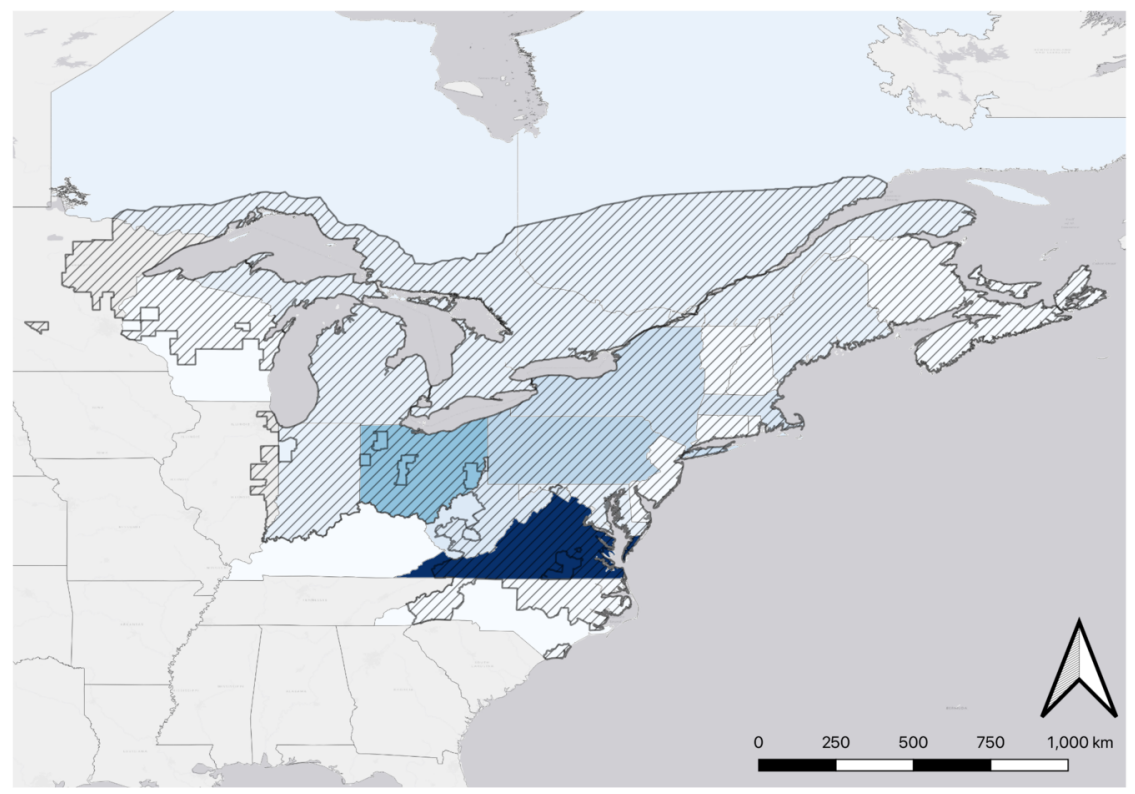

Figure 3. Heatmap of proportion of studies on P. cinereus from different states and provinces since Petranka (1998) was published. Darker shades of blue indicate a higher proportion of studies. Studies that included salamanders from multiple states were counted for each state for a total $\mathrm{N}=448$. Slightly more than half of all studies $(52.2 \%)$ use salamanders from Virginia, Ohio, and Pennsylvania. The geographic range of $P$. cinereus is overlaid as a gray diagonal lines.

Petranka (1998) focuses much of the discussion of geographic variation on ratios of color phenotypes across the range, hypotheses as to the persistence of color polymorphism, and why the color phenotype frequencies vary. Like the population genetic studies, this has been an extremely active area of research in the decades since Petranka (1998), and it has proven to be a complex puzzle. Generally speaking, we have ample evidence 
that the color phenotype frequencies observed prior to 1998 are stable three to five decades later (e.g., through confirmation of earlier records in King et al., 1997; Fisher-Reid et al., 2013; Hantak et al., 2015; Evans et al., 2018); although, see discussion of Grant, Ransom, \& Liebgold, 2018 below). Less clear is the hypothesized relationships between color phenotypes, their physiology, and climate. At the time Petranka (1998) wrote his chapter on $P$. cinereus, there was evidence that striped morphs were more common in cooler climates (i.e., higher latitudes; Test, 1952; Lotter \& Scott, 1977) and evidence that unstriped morphs had a physiology consistent with being more tolerant of warmer conditions than striped morphs (Moreno, 1989). However, more recent studies have identified exceptions to these general patterns and contradictory results that suggest high levels of local variation in both color phenotype frequency (e.g., Hantak et al., 2019) and color phenotype physiologies (e.g., Petruzzi, Niewiarowski, \& Moore, 2006). For example, Petruzzi et al. (2006) found that the patterns of physiological differences between color phenotypes first detected by (Moreno, 1989) in New Jersey were inconsistent and variable across several populations in Ohio. Additionally, Smith, Johnson, \& Smith (2015) found that, while both morphs were equally able to resist water loss, striped morphs recovered from dehydration more successfully than unstriped morphs, particularly in autumn.

Gibbs \& Karraker (2006) show that frequencies of the unstriped morph have increased with sampling year alongside increasing global temperatures, supporting work cited in Petranka (1998) and implying that the unstriped morph may become more common as global temperatures increase due to climate change. However, Moore \& Ouellet (2015), Cosentino et al. (2017), and Evans et al. (2018) show no relationship between color phenotype frequency and sampling year. Cosentino et al. (2017) combine the Gibbs \& Karraker (2006) and Moore \& Ouellet (2015) data sets and find evidence that color phenotype frequency is related to an interaction between land use and temperature: the proportion of striped individuals was positively correlated with forest cover in warmer regions, however, this relationship weakened and disappeared as regional temperatures decreased with increasing latitude. This study suggests that forest cover may buffer natural selection on $P$. cinereus color phenotypes in warmer regions due to global climate change (Cosentino et al., 2017).

Cosentino et al. (2017) note in their discussion, and we agree with their conclusion, that the many studies examining traits between color phenotypes (e.g., Petruzzi et al., 2006; Anthony, Venesky, \& Hickerson, 2008; Davis \& Milanovich, 2010; Fisher-Reid et al., 2013; Smith et al., 2015; Muñoz et al., 2016a; Cosentino \& Droney, 2016) suffer from being limited to a single population or geographic region. To this we add the additional caution that these studies are generally measuring only the surface-active frequency of the color morphs, not the true population-level frequency. Spatial capture-recapture methods, like those used by SPARCnet participants (see below), can better estimate population-level frequencies, but require more consistent and more detailed population monitoring (e.g., Sutherland et al., 2016). Knowing of the existence of geographic limitations in the $P$. cinereus literature from the outset, we recorded the geographic location of each of the 410 studies we reviewed in depth (Table S14; studies with multiple locations counted once for each location; see Methods). Excluding range-wide studies and those with unknown locations, we found that just over half $(52.2 \% ; \mathrm{N}=234 / 448)$ of the studies conducted on P. cinereus since Petranka (1998) are from just three states in the center/southern part of the range: Virginia (29.5\% of studies reviewed), Ohio $(13.2 \%)$, and Pennsylvania (9.6\%; Fig. 3). Nearly one third of the Virginia studies are from Mountain Lake Biological Station (MLBS; $9.2 \%$ of all studies reviewed), a field station in southwestern Virginia, at elevation $(\sim 1100-1200 \mathrm{~m})$, and with its own unique pattern of color phenotype frequencies (Angleberger \& Chinnici, 1975). As we conclude below, our hope is that the distributed nature of SPARCnet participants throughout the range of $P$. cinereus can begin to more robustly address the geographic variation and contradictory results uncovered when exploring differences between color phenotypes.

Fisher-Reid \& Wiens (2015) looked at the maintenance of color polymorphism throughout the genus Plethodon in relation to climate. Striped/unstriped polymorphism is observed in at least eight other species of Plethodon, while another 14 species are fixed for either the striped or unstriped morph (seven species each). All of the polymorphic and monomorphic species are in the three basal groups of Plethodon, suggesting color polymorphism existed within the ancestor of all Plethodon, and has been maintained in the genus for over 40 million years (Fisher-Reid \& Wiens, 2015). Interestingly, there is a strong relationship between color phenotype and climate between species that is similar, but not identical to that observed in $P$. cinereus, but 
there are only two significant within-species climate-morphology relationships among the eight polymorphic species (Fisher-Reid \& Wiens, 2015). Only P. cinereus and a western species, P. vehiculum, show significant climate-morphology relationships, and they are not identical (Fisher-Reid \& Wiens, 2015).

Several studies since Petranka (1998), have explored the variation of color phenotypes in P. cinereus for reasons other than climate, three focusing on selection imposed by predators using clay models (Fitzpatrick et al., 2009; Kraemer, Serb, \& Adams, 2016; Grant et al., 2018), and two focusing on genetic structure and landscape heterogeneity (Fisher-Reid et al., 2013; Hantak et al., 2019). Fitzpatrick et al. (2009) found that avian predators will attack the more common morph when presented with striped and unstriped clay models at varying frequencies, suggesting negative frequency-dependent selection is acting to maintain color polymorphism. However, most populations in the literature have morph frequencies that are not 50:50 striped:unstriped (Petranka, 1998; Cosentino et al., 2017), as would be expected if negative frequency-dependent selection were the only or the predominant factor determining color phenotype frequencies.

Kraemer et al. (2016) used a clay model approach to test for predation selection on color phenotype (striped, unstriped, and erythristic) imposed by mammals. In this study, the authors found complex results driving mammalian predator attacks on the clay models which include both directional selection favoring inconspicuousness and, more strongly, frequency-dependent selection favoring novel coloration. Kraemer et al. (2016) argue that the complexity of how mammals prey on salamanders is strongly influenced by local conditions, and is only one piece of the complex puzzle of morph frequency variation throughout the range of $P$. cinereus.

Grant et al. (2018) combined the clay model approach with demographic data from three years of markrecapture of a Maryland population on the Delmarva Peninsula (i.e., the peninsula includes portions of Delaware, Maryland, and Virginia) to explore morph-specific differences in survival. Delmarva is another location with its own unique color phenotype frequencies (Petranka, 1998). Similar to Long Island, there is a gradient from majority striped in the north, to majority unstriped in the south (Highton, 1977). Grant et al. (2018) found that there was a greater proportion of striped juveniles compared to the proportion of striped adults, suggesting differential survival of the two color phenotypes, with fewer striped individuals reaching adulthood. The authors also found that survival was only explained by the combination of color and age in spring, but not in autumn. Birds were more likely to attack the striped clay models, providing a potential mechanism for the differential survival, while mammals showed no differential predation by color phenotype (Grant et al. 2018). Interestingly, Grant et al. (2018) show color phenotype frequencies that are opposite what Highton (1977) found. In 1977, Highton recorded a color frequency of $\sim 70-80 \%$ striped at the sample locations closest to where Grant et al. (2018) conducted their study. Grant et al. (2018), on the other hand, recorded a color frequency of just $29 \%$ striped over their three years of mark-recapture. This suggests that, unlike other locations which have been sampled repeatedly over time, the color phenotype frequencies on the Delmarva peninsula may not be stable over 40 years. It would be interesting to completely resurvey Highton's (1977) localities and follow Cosentino et al.'s (2017) modeling methods to determine if the same covariates explain the change in color phenotype frequency over time.

Fisher-Reid et al. (2013) and Hantak et al. (2019) are two of several recent studies mentioned briefly above which have tried to leverage the technological advances in genetics since Petranka (1998) to understand variation color phenotype frequencies in P. cinereus. Williams, Highton, \& Cooper (1968) were the first to note that the typical color morph ratio (70:30 striped:unstriped) is not observed on Long Island, New York. Western Long Island is $100 \%$ striped, southeastern Long Island is $100 \%$ unstriped, and the center and northeastern parts of the island are roughly 50:50 striped:unstriped (Williams et al., 1968; FisherReid et al., 2013). Using microsatellite markers, Fisher-Reid et al. (2013) found that the $100 \%$ unstriped populations are genetically isolated from the rest of the Long Island P. cinereus populations (as measured by $\mathrm{F}_{\mathrm{ST}}$ ), and salamanders at these localities also show significant differences in morphology, microclimate, and macroclimate. Fisher-Reid et al. (2013) documented a soil moisture gradient on Long Island that correlated with the change in morph frequency, suggesting selection may have played a role in driving the less fit morph extinct at the gradient extremes. In northern Ohio, Hantak et al. (2019) focus on the population genetics from 28 localities which span a range of color phenotype frequencies from $100 \%$ striped in eastern 
Ohio to nearly $100 \%$ unstriped in western Ohio. Using microsatellite markers, they find a complex interaction between gene flow, genetic drift, and natural selection working together to explain morph frequencies, similar to Kraemer et al. (2016). Using ecological niche modeling, Hantak et al. (2019) found waterways were the most important factor in explaining morph frequency variation, likely, as the authors suggest, because they are strong dispersal barriers.

Taken together, these many studies exploring color polymorphism in $P$. cinereus make a strong case for complex interactions between macroclimate, microclimate, forest type, and predators on variation in color phenotype in $P$. cinereus. We refer the reader to Hantak et al. (2019)'s discussion on the complex maintenance of color polymorphism in $P$. cinereus for additional thoughts on the complexity of color polymorphism in $P$. cinereus. Our search recovered two additional studies that explored geographic variation not related to color phenotypes: one focused on skin microbial communities (Muletz-Wolz et al., 2017) and the other on phylogeographic patterns of pheromone profiles (Kunkel et al., 2019). Muletz-Wolz et al. (2017) is discussed in more detail below in our section below on Microbiota and Disease, however in the context of geographic variation, they sampled the skin microbial communities of $37 P$. cinereus individuals across three localities in the central Appalachians, one locality encompassed an elevation gradient. As other microbial studies have found (see below), they found high levels of microbial diversity, and little overlap from one location to another, suggesting high taxonomic turnover of microbial communities across the landscape (Muletz-Wolz et al., 2017). Kunkel et al. (2019) compared the pheromone profiles of striped males from the Ohio (OH) clade to striped males from the Pennsylvania (PA) clade (Radomski et al., 2020) across a gradient in northern Ohio (overlapping the study area of Hantak et al., 2019). They found that while pheromone profiles differed between populations, they did not differ between phylogeographic clades. This suggests that the two clades, $\mathrm{OH}$ and $\mathrm{PA}$, are not yet reproductively isolated from each other.

\section{(c) Distribution and Adult Habitat}

Petranka (1998) gives a brief description of the geographic range of $P$. cinereus, as well as the preferred habitat of adult salamanders. He notes that $P$. cinereus adults can be found in deciduous, northern conifer, and mixed deciduous-conifer habitats throughout their range, but reach their greatest densities in welldrained, mature forests with deep soils. He also notes that populations are absent or at low densities in highly acidic soils, perennially wet soils, and shallow, rocky soils (Petranka, 1998).

In compiling the more recent literature on the distribution and adult habitats of $P$. cinereus we noted 25 sources which simply document that $P$. cinereus was present at a given study site and categorized these as Regional Herpetofaunal Lists (Table S11). In reviewing these sources, it is clear that the geographic distribution of $P$. cinereus has remained stable over the decades of intensive study, and if anything, it is underestimated by the most commonly cited range maps. That said, we only found two sources explicitly documenting new records of $P$. cinereus. The first, Placyk, Seider, \& Gillingham (2002), documented $P$. cinereus from two islands in Lake Michigan that had not been previously inventoried. Island populations of $P$. cinereus are common, particularly in areas that were formerly glaciated (e.g., Long Island, Martha's Vineyard, Maine coastal islands, Canadian provincial islands; VertNet, 2021). The second recorded a new, high latitude elevation record for $P$. cinereus, found at $1173 \mathrm{~m}$ in Maine, which is considered subalpine (Willey \& Jones, 2010). Only three studies noted fewer than expected (Silva et al., 2003; Meshaka et al., 2019) or no (Hartzell, 2019) P. cinereus in suitable habitat, however all of these studies focused their sampling during the summer months, when the heat drives the vast majority of $P$. cinereus underground, thus we doubt they represent true absences or declines in density (Fig. 4). Silva et al. (2003) does note that prior work has shown P. cinereus is generally rare on Prince Edward Island, Canada, however a survey in October or April would provide a clearer picture of the actual abundance. In general, the autumn season, particularly October, has the highest detection rates (e.g., Leclair, Levasseur, \& Leclair, 2008; McGhee, 2013; Sutherland et al., 2016), while in the far southern part of the range, salamanders are surface-active from autumn through the winter months into spring (Becker et al., 2013; Hernández-Pacheco et al., 2019; Fig. 4). 
More of the work since (Petranka, 1998) has focused on the microhabitat preferences of both adult and juvenile salamanders, particularly with regards to sampling methods using natural vs. artificial cover objects (NCOs vs. ACOs). We categorized more than 50 studies as focusing on adult and/or juvenile habitat, as well as an additional 19 studies which test a variety of sampling methods based on presumed habitat preferences. Of those latter 19 studies, 18 are focused on ACO design, ACO survey performance compared to NCO and leaf litter surveys, and ACO best practices. Generally speaking, ACOs are a well-vetted method for standardized sampling of $P$. cinereus, one which SPARCnet employs. Indeed, Hesed (2012) argues that ACO methodology has been optimized for $P$. cinereus over other woodland salamander species. Among our reviewed studies, at least one found ACOs are more efficient than leaf litter surveys (e.g. Otto \& Roloff, 2011) and several found ACO surveys are comparable to NCO surveys (e.g., Monti, Hunter, \& Witham, 2000; Grover, 2006; Moore, 2009; Ciul et al., 2010).

In terms of best practices, wood boards (MacNeil \& Williams, 2013), resting directly on the ground (Carfioli et al., 2000), and sampled no more frequently than once per week (Marsh \& Goicochea, 2003; Otto, Bailey, \& Roloff, 2013) have the highest encounter rates. ACOs are colonized by salamanders within 2 months of installation (Bonin \& Bachand, 1997), and there does not seem to be much impact on occupancy under ACOs based on the type of wood used (Bennett et al., 2003; Moore, 2005) or the age of the ACO (Monti et al., 2000; Carlson \& Szuch, 2007). That said, recent SPARCnet work has shown ACO age to be important, with older boards capturing more salamanders than younger boards (Hedrick et al., 2021). In Virginia, one study found ACOs may be biased toward attracting adult salamanders and exclude juveniles compared to NCOs (Marsh \& Goicochea, 2003), but other studies from Maine and Québec found no age/size differences between ACO and NCOs (Monti et al., 2000; Moore, 2009). Siddig, Ellison, \& Jackson (2015) found that ACO surveys overestimate the true density of salamanders, while NCO surveys underestimate the true density, when true density was estimated from removal. However, the sampling in Siddig et al. (2015) was done in July in Massachusetts, when surface densities are at or approaching their lowest (see below and Fig. 4), and so should be repeated during the autumn or spring surface-active seasons for accuracy. Work by SPARCnet members has found that, while estimates of space use and detectability are both sensitive to the spacing and extent of an ACO array, estimates of population density are robust to both spacing and extent (Fleming et al., 2021).

Beyond comparisons of ACOs vs. NCOs, several studies find that while you can find P. cinereus in the leaf litter, they are more likely to be encountered under cover objects (Moore et al., 2001; Otto \& Roloff, 2011; McGhee, 2013). Preference for woody NCOs vs. rocky NCOs varies by study location. In Ohio, woody NCOs were preferred (Iverson \& Smith, 2010), while in Vermont, rocky NCOs were preferred (Richmond \& Trombulak, 2009). Positive correlations between cover object size (either diameter or area) and salamander size (mass, snout-vent-length) are inconsistent in those studies which have explored the relationship; e.g., Moore et al. (2001) and Strojny \& Hunter (2010a) find these correlations, while others found no relationship between cover object size and salamander size (Faragher \& Jaeger, 1997; Moore, 2005, 2009).

A study in Vermont by McKenny, Keeton, \& Donovan (2006) suggests that structural complexity of the forest floor is important, finding $P$. cinereus to be more abundant when there was well-decayed coarse woody debris compared to less-well-decayed coarse woody debris. Strojny \& Hunter (2010a) suggest that large coarse woody debris is important based on their study in Maine finding salamanders to be more abundant under logs of larger diameter in canopy gaps after timber harvest. Leaf litter preferences are inconsistent within the same study site, suggesting that individual preferences are highly variable over time (Iverson \& Smith, 2010; Renaldo et al., 2011). Iverson \& Smith (2010) found no preference for deciduous vs. pine leaf litter, while Renaldo et al. (2011) found a preference for deciduous over pine, and the strongest preference for a combination of deciduous and pine litter.

Several studies directly contradict Petranka (1998)'s notes that $P$. cinereus is uncommonly found on perennially wet or highly acidic soils: Chalmers \& Loftin (2010) regularly observed P. cinereus in wetland habitats in Maine, and several studies have found $P$. cinereus in riparian environments (e.g., Moore et al., 2001 in Pennsylvania; Anderson, Solis, \& Osbourne, 2013 in West Virginia, and McGhee, 2013 in Virginia). 
In particular, Anderson et al. (2013) and McGhee (2013), both found equal encounter rates for P. cinereus between riparian and upland habitats. Moore \& Wyman (2010) found a seemingly healthy population of $P$. cinereus in an extremely acidic forest in Québec, Canada. There, the $\mathrm{pH}$ under coverboards ranged from 3.1 to 5.2 , and $82 \%$ of captures over five years were from coverboards with a soil $\mathrm{pH}$ [?] 3.8. The Quebec population also had larger salamanders (both in body size and mass; Moore \& Wyman, 2010) compared to other studies, directly contradicting an earlier study which suggests low $\mathrm{pH}$ substrates cause a loss of body mass (Frisbie \& Wyman, 1991). Similarly, Bondi et al. (2016) found P. cinereus to occupy a wider range of soil $\mathrm{pH}$ than earlier studies, five of their 34 sites in northern New York, Vermont, and New Hampshire had $\mathrm{pH}<3.0$ and seemingly healthy $P$. cinereus populations. They found no evidence that $P$. cinereus was selecting microhabitats preferentially by $\mathrm{pH}$ (Bondi et al., 2016). While these low $\mathrm{pH}$ values may be tolerable to $P$. cinereus, at least one recent study suggests they prefer more neutral soil $\mathrm{pH}$. In Ontario, Gorgolewski et al. (2016) added wood ash to forest floors to raise the $\mathrm{pH}$ from $\sim 5$ to $\sim 6.5$ and found that the abundance of $P$. cinereus increased under coverboards with ash compared to those without ash.

Three additional sources found P. cinereus in novel environments. Hughes, Petersen, \& Duffield (1999) found one salamander dead inside a pitcher plant (Sarracenia purpurea) in two different locations: West Virginia and Pennsylvania. Both sites were the typical bog habitat of $S$. purpurea, which is an unusual place to find woodland salamanders. Hughes et al. (1999) hypothesize these two individuals were foraging when they died, given their stomach contents and the abundance of arthropod prey in the bog. Roble (1999) found an unstriped individual under driftwood on the sandy bank of the Potomac River in Virginia. Finally, LeGros (2018) found an individual hiding under the lid of a backcountry privy in Ontario, possibly feeding on the flies attracted to the privy. LeGros (2018) displaced the individual from the privy, and it returned within 9 hours. Individual identification was confirmed using photographs of the spotting pattern on the head and tail (LeGros, 2018). To these observations we add two novel environment observations by SPARCnet members, both in Maryland: a gravid female under an old tire in a suburban backyard, and a salamander more than a meter underground found while installing a flagpole in a different suburban yard (E.H.C. Grant; pers. comm.)

Many studies attempt to characterize the preferred microhabitat of $P$. cinereus, either by measuring covariates in the field where salamanders are present or by doing behavioral choice trials of different habitats in a laboratory setting. In Virginia, Mitchell et al. (1997) found deep soils to be important in predicting $P$. cinereus presence in four out of five forest stands. In the laboratory, individuals from an Ohio population preferred high $\mathrm{pH}$, shaded habitat with medium-high moisture (Sugalski \& Claussen, 1997). The authors were able to show that $\mathrm{pH}$ was most influential in preferences, and that the combination of acceptable soil $\mathrm{pH}$ and moisture levels made a wider range of light intensities acceptable. In Pennsylvania, Brenner, Smith, \& Marr (2005) found the presence of $P$. cinereus was correlated with soil moisture and temperature, and indirectly correlated with litter depth. Not surprisingly, given the species is fully terrestrial, they also found that $P$. cinereus presence was not related to either distance or direction from vernal pools (Brenner et al., 2005). In the field, Farallo \& Miles (2016) compared the microhabitat characteristics of $P$. cinereus to two microendemic species, P. sherando and P. hubrichti in Virginia. They found evidence for active seeking-out of preferred habitats, as measured by temperature and moisture levels of the air and soil in all three species, as well as some evidence of microclimatic niche differentiation between all three species, suggesting character displacement when P. cinereus is sympatric with the microendemic species (Farallo \& Miles, 2016). A follow-up study on the same three species by Marsh et al. (2019) found that peak activity temperatures for $P$. cinereus were similar to $P$. sherando and cooler than $P$. hubrichti. There were no significant moisture patterns detected. As expected, the wide-ranging $P$. cinereus had a much larger range of active temperatures compared to P. hubrichti, but not when compared to P. sherando (Marsh et al., 2019). Yu et al. (1998) found that $P$. cinereus from Pennsylvania had high levels of constitutive heat shock protein 70; levels which did not change with acclimation to different temperatures in the laboratory. They compared $P$. cinereus to southern congener, $P$. jordani, and found that P. jordani was not as tolerant of heat shock as $P$. cinereus (Yu et al., 1998). In Ontario, Luymes \& Chow-Fraser (2019) found populations to be stable over an 18-year sample period with an average temperature of $12 \mathrm{oC}$ during the spring and autumn active seasons. This study 
also argues that monitoring programs which hope to detect population declines should plan on sampling for at least a decade in order to more accurately estimate occupancy (Luymes \& Chow-Fraser, 2019).

Kolozsvary \& Swihart (1999) explored the relationship between patch size and P. cinereus occurrence in Indiana, finding a strong positive relationship as forest size increased from 1 ha to 1000 ha. A regression modeling study based on presence/absence data from a location in northern Virginia also found $\mathrm{pH}$ variability to be important in detecting $P$. cinereus (McGhee \& Killian, 2012), suggesting salamanders actively select "good" microhabitat patches within less desirable patches. However, their sample size for their presence data was very low ( $\mathrm{N}=42$ salamanders over 2 years; presence detected on $29 \%$ of transects) compared to their absence data. McGhee \& Killian (2012) argue that at their location, soil pH may be more important in determining which habitats salamanders avoid (low $\mathrm{pH}$ with low $\mathrm{pH}$ variability). In Ontario, Brown et $a l$. (2020) found that understory characteristics better predicted presence of $P$. cinereus than more macroscale variables like climate, disturbance, canopy cover, or landscape.

Finally, a handful of studies have explored how social hierarchies may be involved in habitat choices in $P$. cinereus. Forester \& Anders (2000) found that hatchlings are more likely to associate with substrates marked by their mother than they are with substrates that are just wet or marked by a non-relative conspecific female. Hurst \& Smith (2006) found that females will avoid areas with cues from five or more conspecifics, while males do not discriminate. These two studies as well as much of the work detailed in Jaeger et al. (2016) imply that $P$. cinereus can not only recognize kin vs. non-kin, and conceptualize the number of individuals in an area, but use this information to inform movement decisions. In West Virginia, Riedel, Russell, \& Ford (2012) found that juveniles may actively avoid or be unable to compete for limited cover in non-forested habitats (e.g., meadows, pastures) that adults appear to live in without issue.

\section{(d) Breeding and Courtship}

Petranka, (1998) splits P. cinereus reproductive biology into two sections: Breeding and Courtship, which details all male and female behavior prior to insemination, and Reproductive Strategy, which focuses solely on female behavior after insemination. We follow a similar structure here. Petranka (1998) first describes the remarkably long mating season in $P$. cinereus based on studies that document the presence of sperm in female cloacae from autumn through winter to early spring. Mating is believed to occur primarily in the autumn. Breeding frequency is annual for males, while females breed biennially in most populations, particularly in more northern portions of the range where the active season is shorter. That said, Petranka (1998) notes that there is some evidence that larger females can breed annually even in more northern populations (> $44 \mathrm{~mm}$ SVL in Connecticut; Lotter, 1978). Courtship follows behaviors stereotypical of other plethodontids (Arnold, 1977), including pheromone signaling, male abrasion of females with enlarged premaxillary teeth, nose tapping, and a coordinated tail-straddle walk, during which males deposit a spermatophore on the ground that females pick up by walking over it. It had also been observed that intruder males could disrupt courtship by biting the courting male. Lastly, (Petranka, 1998) described a fecal pellet squashing behavior by gravid females as a mechanism for selecting mates with higher diet quality. Much of the related behavioral work on P. cinereus has previously been well-reviewed in Jaeger et al. (2016).

Extensive work has been done to characterize the sexually dimorphic glands used in courtship by $P$. cinereus from the same population in Indiana. Sever (1997) observed that females usually store sperm for three to four months, and up to nine months if mating occurred right before the winter hibernation period, however, contrary to other locations and Petranka (1998), autumn mating was rare in this population. A followup study described the complex spermatheca of females, where sperm clusters occurred in parallel arrays (Sever \& Siegel, 2006), and also confirmed the predominance of spring mating in this population based on absence of sperm in females collected in October compared to those collected in April. Sever \& Siegel (2015) found seasonal differences in the size and secretory activity of caudal courtship glands in males, with the greatest size and secretory activity occurring in April and October. Mental glands in males show similar seasonal changes in size and secretory activity (Sever, 2017). Related to these patterns, Church \& Okazaki 
(2002) found that plasma testosterone levels and testes mass both vary seasonally in males from Virginia. Testosterone levels peak in the autumn in early October and reach their lowest point at the end of the spring in May. Peak testes mass is offset from testosterone, peaking in mid-summer (July) and reaching its lowest point in the winter hibernation season (November - March).

In Virginia, Simons, Felgenhauer, \& Thompson (1999) identified and described several mucous and serous glands in the shoulder and postcloacal region of P. cinereus. This study was followed by Hecker et al. (2003) in New York, who focused on the postcloacal mucous and two types of serous glands (S1 and S2). Hecker et al. (2003) hypothesized that the S1 serous glands are for scent-marking while the S2 serous glands are used in nutrient storage and defense. More recently, there has been a focus on the pheromones used during courtship. Wilburn et al. (2014) notes the presence of three pheromones: the plethodontid receptivity factor (PRF), the plethodontid modulating factor (PMF), and the sodefrin precursor-like factor (SPF). They hypothesize that $\mathrm{PRF}$ is replacing SPF in P. cinereus due to only trace amounts of SPF being found. It is also interesting to note that while $P$. cinereus has evolved to produce PRF, it has not evolved to utilize the newer olfactory method to deliver courtship pheromones. As noted above, Kunkel et al. (2019) found population-level differences in pheromone chemistry, but not enough to prevent gene flow across two phylogeographic clades.

At the northern range limit, Leclair, Levasseur, \& Leclair (2006) and Leclair et al. (2008) have documented a much more delayed reproductive phenology in $P$. cinereus, particularly in females. In a Quebec population, males become sexually mature between 3-5 years old and reproduce annually, while females were mostly unable to reproduce until 4-6 years old (Leclair et al., 2008), much later than earlier studies conducted in more southern populations (Petranka, 1998). The decreased growth rate in northern populations is possibly due to colder temperatures and more limited opportunities for growth (Leclair et al., 2008). Both studies aged individuals using skeletochoronology, and found that these northern females typically only live 8 years and it takes three years for a clutch of eggs to mature (Leclair et al., 2006, 2008). Therefore, most female $P$. cinereus in this population only reproduce once in their lifetime. Leclair et al. (2008) also found the juvenile sex ratio in this Canadian population was female-biased, likely due to the delayed female maturity and rare subsequent reproduction.

Male-female pairs are often observed sharing cover objects in $P$. cinereus populations, especially during the courtship season, while female-female pairs were uncommon and male-male pairs were very rare (Peterson $e t$ al., 2000). Peterson et al. (2000) hypothesizes same-sex pairs occur coincidentally due to limited territory space. Meche \& Jaeger (2002) found no evidence for males preferring tail-intact over tail-autotomized females. In Ohio, Anthony et al. (2008) and Acord, Anthony, \& Hickerson (2013) both observed that male-female pairs are usually the of same color phenotype, suggesting positive assortative mating by color. However, a follow-up study by Jaworski et al. (2018) found that striped males cannot differentiate between female color phenotypes and thus showed no preference for either striped or unstriped females, but males did show preference for larger females. Preference for larger female size by males was also observed in Anthony et al. (2008) and Acord et al. (2013). There is some evidence that P. cinereus follow social monogamy for a mating strategy. Several articles (e.g., Guffey, MaKinster, \& Jaeger, 1998; Gillette et al., 2000; Jaeger et al., 2001; Jaeger, Gillette, \& Cooper, 2002) support this, noting that males and females exposed to unfamiliar individuals (and their scents) behave more aggressively than when exposed to familiar or partner individuals. Males and females were observed to be more aggressive towards polygamous partners than monogamous partners or strangers (Jaeger et al., 2016). Using this evidence, Jaeger et al. (2002) hypothesizes that $P$. cinereus uses sexual coercion against polygamous partners. For a thorough review of the behavioral work on P. cinereus, particularly as it pertains to courtship and territoriality, we recommend Jaeger et al. (2016).

Atypical courtship behaviors have also been observed in P. cinereus, although their relevance to the breeding and courtship of this species is unclear. In Virginia, Dyal (2006) observed a female-first tail straddle walk (fftsw), not observed in two other plethodontids studied in the same paper. However, data suggested that fftsw did not lead to successful spermatophore deposition in P. cinereus and therefore Dyal's (2006) hypothesis that females have a larger role in courtship than previously thought is difficult to evaluate. Courtship behaviors in plethodontids more generally was recently reviewed by Staub, Stiller, \& Kiemnec-Tyburczy 
(2020). They specifically describe a more active role for females in courtship than historical studies would suggest, a pattern not unique to salamanders as EEB fields achieve greater gender parity in both scientists and their science (Staub et al., 2020).

Several studies since Petranka have further investigated the fecal pellet squashing behavior. Karuzas, Maerz, \& Madison (2004) found that while all females investigated male-marked substrates more than female ones, females on poor diets were especially likely to participate in the pellet-squashing behavior. Karuzas et al. (2004) agree with Petranka (1998) that the behavior is used to assess conspecifics' diets, but hypothesize that the behavior is not limited to the context of mate selection. Chouinard (2012) suggests that fecal pellets may also not be necessary for assessing diet quality of potential mates. In a laboratory setting, Chouinard (2012) observed that males on high quality diets had more protein in the mental gland compared to males on low quality diets. Females responded more quickly to and spent more time with males fed high quality diets (Chouinard, 2012).

We still do not know what influences paternity, but females are known to be able to store multiple spermatophores. We also do not know how the sperm from different males interact. There are additional gaps in our knowledge surrounding how/why males and females may occupy the same cover object, and if there is any geographic variation in social behavior (almost all of the research surrounding mating strategies has been done with individuals from MLBS). Related to this, more field research should be performed in order to determine if long-term pairing of males and females over multiple seasons occurs. Further research should also focus on mate selection in $P$. cinereus, specifically identifying the role of visual stimuli. Finally, we are unsure of the specific function of courtship pheromones, especially PRF and PMF, since previous research assumes they perform similar roles in $P$. cinereus as in other tested plethodontids.

\section{(e) Reproductive Strategy}

In this section, Petranka (1998) summarizes what was known at the time about $P$. cinereus oviposition, parental care, and basic development. Generally, females oviposit in late spring or early summer, although Petranka (1998) reported observations of oviposition as late as August in northern Michigan. Nests are made in cavities or crevices that females, and sometimes males, attend while brooding. Females aggressively defend eggs from conspecifics and predators, and there is evidence for reduced survivorship of abandoned clutches. During brooding, females have limited opportunities to feed and grow significantly less, a pattern which is exacerbated in the presence of another female (Petranka, 1998). However, females frequently cannibalize their own eggs when induced to oviposit in a lab and eat the eggs of abandoned clutches in both laboratory and field observations. Petranka (1998) notes that northern or high elevation females tend to oviposit in cavities within decaying logs or beneath rocks and logs, while oviposition in southern populations was typically observed in subsurface retreats created by other animals. Petranka (1998) describes the gross anatomy of eggs and their arrangement in the clutch, generally suspended from the roof of the cavity in a grape-like cluster. Petranka (1998) also reports clutch sizes and ovarian egg counts from several locations, with an average clutch size between 6-9 eggs. In three populations where it was explored, the number of mature eggs was correlated with female SVL (Connecticut: Lotter, 1978; Tennessee: Nagel, 1977) or female body mass (New York; Fraser, 1980). Egg incubation lasts for approximately six weeks, and Petranka (1998) reported several populations where hatching occurs in August or September. During the time of Petranka (1998), very little was known about the development of embryos, but mature embryos had been observed with three gills on either side of the head that are lost around the time of hatching.

Since $P$. cinereus does not oviposit naturally in the lab (laboratory oviposition is induced via hormone injection, typically with agonists of luteinizing hormone-releasing hormone (LHRH); e.g., Evans et al., 2020), and their nests are often difficult to locate underground, studies on their reproductive strategy since Petranka (1998) have been limited. There have been several important studies, however, which document key oviposition and care behaviors and further characterize the development of $P$. cinereus eggs and embryos. Oviposition dates, when reported, generally match what was reported in (Petranka, 1998), occurring fre- 
quently around June (Piersol, 1910; Burger, 1935; Bishop, 1941; Lynn \& Dent, 1941; Sayler, 1966), although a nest in Virginia was found as early as April (Orr \& Ernst, 2005). Madison, Wareing, \& Maerz (1999) described the exact ovipositing behavior of females induced to lay eggs in the lab. Females assume an upside down S-shape to lay eggs, using their tail to brace themselves, and were in this position for over 6 hours. From this position, females suspended their eggs from the base of a log, and adjusted the positioning of the eggs after oviposition with her snout. Madison et al. (1999) hypothesized that this would place tailless females at a disadvantage for ovipositing, and more recent work has observed tailless females to produce fewer ova, indicating that reproductive success is inhibited by tail loss (Yurewicz \& Wilbur, 2004). Madison et al. (1999) also hypothesize an evolutionary advantage for egg suspension, since unsuspended eggs were cannibalized $100 \%$ of the time, while suspended eggs were only cannibalized $50 \%$ of the time.

While controlled breeding is not generally possible in the lab (Petranka, 1998), cross-fostering experiments are possible. Crespi \& Lessig (2004) successfully cross-fostered 15 clutches, with 10 control clutches (eggs removed from mother's care and then replaced). This study showed the presence of maternal effects, likely driven by differences in parental care: there was a positive correlation between a foster mother's SVL and hatchling's body length. Behaviorally, larger mothers did not move their eggs as often, and spent more time in direct contact with their eggs (Crespi \& Lessig, 2004).

Kerney (2011) created a complete larval staging table for P. cinereus, showing the development of embryos over 72 days until hatching. Despite being a direct developing species, $P$. cinereus embryos were found to have many characteristics indicative of a metamorphosing species, including hyobranchial skeleton development and the temporary presence of certain carteliges, gill clefts, and Leydig cells (Kerney, 2011; Kerney et al., 2012). This is relevant to the loss and re-evolution of the larval stage by plethodontid species in the genus Desmognathus, as this shows that the development of key larval traits was not fully lost in directdeveloping plethodontids (Kerney et al., 2012).

Brooding females were more likely to abandon clutches in low-resource conditions (Yurewicz \& Wilbur, 2004). The authors found that smaller clutch sizes were not correlated with larger eggs, making clutch size an appropriate proxy for reproductive investment. Yurewicz \& Wilbur (2004) also found that females were less likely to abandon or cannibalize larger (about eight eggs) clutches, indicating that the high energetic cost of brooding outweighs the fitness value of smaller (about five eggs) clutches. However, another study showed that brooding females defended large (ten eggs) and small (four eggs) clutches with equal aggressiveness (Tornick, 2010). These results seem contradictory about the ability of females to differentiate between clutch sizes and make energetic investment decisions accordingly, but the discrepancy may be due to the failure of Tornick (2010) study to investigate female behavior with clutches of fewer than four ova. Females were more aggressive when defending more mature clutches, indicating that the developmental stage of eggs influences investment decisions by brooding female P. cinereus (Tornick, 2010). Ovarian egg counts were also used to determine the average clutch size of some populations, as these observations are typically more accessible and non-invasive. However, no studies have yet determined the relationship between ovarian egg counts and actual clutch size. Since $P$. cinereus are known to reabsorb eggs (Ng \& Wilbur, 1995), is it unclear how reliable this method is for predicting clutch size.

Future research on the reproductive strategy of $P$. cinereus females should be cognizant of potential behavioral changes due to laboratory settings (e.g., inducing oviposition increases oophagy; Petranka, 1998; Madison et al., 1999). More field-based studies and observations of reproduction and brooding are necessary for confidence in the results from laboratory studies. Future studies should also determine the accuracy of using ovarian egg counts to predict clutch size, as gravid females are much easier to capture than nesting females. We still do not know what factors influence reabsorption of eggs, nor what factors induce females to cannibalize their own eggs. Finally, although reproductive timing and clutch size have been shown to be influenced by latitude, there have not been any recent studies investigating reproductive trends in populations at the southern range limit and our knowledge of patterns at the northern range limit is from a single population (Leclair et al., 2006, 2008). Those southern populations which have been studied are at elevation (i.e., at MLBS; Crespi \& Lessig, 2004; Yurewicz \& Wilbur, 2004), and have their own local phenology distinct 
from the both southern and northern low elevation populations (Fig. 4), suggesting their results may not be generalizable to those regions.

\section{(f) Terrestrial Ecology}

The terrestrial ecology section is the most lengthy section of Petranka (1998). When categorizing studies, we found it useful to use a finer scale breakdown of topics within terrestrial ecology, and these are represented by our subheaders in this section. Briefly, Petranka (1998) covers the following topics in the following order in his terrestrial ecology section: diet, juvenile habitat and development, density estimates and habitat preferences, feeding behavior, surface activity timing and behavior, and social behaviors and territoriality. There are some overlapping topics with other sections, for example juvenile habitat can just as easily be discussed in the Distribution and Adult Habitat section above, which we opted to do, as well as some spatially disjunct topics within Petranka (1998) which we have opted to combine (i.e., diet and feeding behaviors). Finally, we end this section with a catchall section on studies of general behavior not directly related to any of the preexisting categories in this or other sections.

\section{(i) Diet and Feeding Behavior}

Petranka (1998) described the diet of $P$. cinereus as any palatable metazoan prey an individual can capture and that, while variable, there are not noticeable shifts in dietary preferences as individuals grow. Prior observations indicated that adults can be cannibalistic, consuming eggs and juveniles in both the lab, and on rare occasions, in the field. It was thought that $P$. cinereus fed primarily on the forest floor, but Petranka (1998) also notes that individuals have been observed to climb vegetation to forage. There was also limited evidence for winter feeding in underground retreats at a population in Indiana, based on the stomach contents of specimens dug out of abandoned ant mounds (Petranka, 1998). Adult P. cinereus were known to use both visual and olfactory cues to find prey and had been observed selecting higher quality (i.e., higher caloric value) prey over low quality prey when prey density is high, but eating all available prey when prey density was low (Petranka, 1998).

At least 34 recent studies have focused on the diet and/or feeding behaviors of $P$. cinereus since Petranka (1998). Diet was assessed directly by examining stomach contents (via dissection or flushing) in 14 studies. As might be expected, prey items vary by location, but ants, mites, annelid worms, and springtails (Collembola) are the most common prey items across the range (Bellocq, Kloosterman, \& Smith, 2000; Rooney, Antolik, \& Moran, 2000; Adams \& Rohlf, 2000; Maerz \& Karuzas, 2003; Maerz et al., 2005; Maerz, Myers, \& Adams, 2006; Arif, Adams, \& Wicknick, 2007; Anthony et al., 2008; Ivanov et al., 2011; Bondi, Green, \& Beier, 2015; Bondi et al., 2019; Paluh et al., 2015; Stuczka, Hickerson, \& Anthony, 2016; Hantak, Paluh, \& Hickerson, 2016). Interestingly, one study based in Delaware found an abundance of Collembolla in the field, but none in salamander stomachs, although the sample size of individual stomach contents was small compared to other studies listed above $(\mathrm{N}=12$; Rooney et al., 2000). Three studies explored relationships between $P$. cinereus and non-native prey, finding evidence that non-native invertebrates can either serve as an added resource consumed alongside native prey (Maerz et al., 2005; Ivanov et al., 2011) or are ignored if deemed unpalatable (Ducey et al., 1999). While by no means the first study to use the method, Bondi et al. (2015) tested the efficacy of the gastric lavage method (i.e., stomach flushing) as a technique for non-lethal sampling of salamander stomach contents. The authors find it to be reliable, $95 \%$ of salamanders regurgitated some or all of their stomach contents. Large prey (land snails, adult Coleoptera) were the least likely to be recovered through gastric lavage, and often these instances were singular items left behind while all smaller prey were recovered (Bondi et al., 2015). Finally, there were two studies which provide additional confirmation of cannibalism in the field (Maerz \& Karuzas, 2003; Gade et al., 2017). 
Several studies have explored head morphology in P. cinereus and other congeners (e.g., Adams \& Rohlf, 2000; Maerz et al., 2006; Arif et al., 2007; Adams, 2011), exploring questions of character displacement in sympatry with congeners and relationships between head shape and prey size. These are all discussed in more detail in the Community Ecology: Other salamanders and P. cinereus Interactions section below. Here, we note that Adams (2011) explored the heritability and quantitative genetics of head shape variation in hatchling $P$. cinereus and $P$. nettingi. The heritability of head shape was generally high $\left(\mathrm{h}^{2}>0.65\right)$, however, Adams (2011) also found evidence that these two species would evolve differently in response to the same selection pressure on head shape, suggesting head shape as a potential trait axis by which diversification in salamanders occurs.

Foraging behaviors, including territoriality and general foraging activity, has also been studied in detail. Maerz \& Madison (2000) is a complementary field-based study to the primarily lab-based studies of Jaeger et al. (2016) which shows that territoriality is correlated to food density. They found that body size was positively correlated with both total number and volume of prey when prey was at low density. When prey was at high density, there was lower shelter fidelity, and greater same-sex cohabitation of cover objects among females (Maerz \& Madison, 2000). Anthony et al. $(2008,2017)$ explore the relationship between diet and color phenotype in Ohio. Anthony et al. (2008) found that striped morphs eat more diverse and more profitable (easier to digest) prey than unstriped individuals, while the follow-up study found that striped morphs resided on territories with a greater abundance of available prey items (Anthony et al., 2017). Together with a related study from the same population on morph-specific aggression by Reiter, Anthony, \& Hickerson (2014), these three studies suggest that striped morphs may competitively exclude unstriped morphs from territories with larger quantities of more energetically profitable prey. In contrast, at a different Ohio locality, Hantak et al. (2016) found no significant differences between the diets of sympatric striped and erythristic morphs.

More recent work confirms that $P$. cinereus forage at all times of day, although night foraging is likely the predominant mode (Placyk \& Graves, 2001a; Liebgold \& Dibble, 2011). Night-active salamanders had higher mass, and there was lower prey abundance in their field enclosures, compared to enclosures with less night-active salamanders (Liebgold \& Dibble, 2011). At night, olfactory cues are thought to be most important, as evidenced by increased nose tapping in the dark (Placyk \& Graves, 2001a), and decreased predation efficiency when the vomeronasal system is impaired (Placyk \& Graves, 2002). Telfer \& Laberge (2013) found that salamanders were more likely to find and investigate soluble olfactory cues over volatile cues. The reliance on soluble olfactory cues is also supported by Maerz, Panebianco, \& Madison (2001), which found that foraging activity was reduced when exposed to water soiled by a garter snake. Social hierarchies also appear to influence foraging activity. Liebgold \& Dibble (2011) found that both adults and juveniles foraged more actively when enclosed with familiar individuals of the opposite age class compared to when enclosed with unfamiliar individuals of the opposite age class.

Gibbons, Ferguson, \& Lee (2005) found that while there is a genetic component to foraging efficiency, learning and exposure to prey can improve foraging efficiency over time. Petranka (1998) noted that climbing behavior was likely a foraging tactic, and the results of Cote et al. (2016) seem to confirm this. They found that salamanders exposed to dowels with prey scent trails climbed higher and for longer than on control (unscented) dowels. Individuals experimentally infected with the fungal pathogen Batrachochytrium dendrobatidis $(B d)$ appear to compensate for this infection by foraging more in a laboratory setting (Hess et al., 2015). The authors found minimal fitness effects of $B d$ infections compared to uninfected controls, suggesting that the extra foraging did help those individuals fight infection. Finally, a small number of studies explore interference competition between $P$. cinereus and non-salamander predators: centipedes (Hickerson, Anthony, \& Walton, 2012) and spiders (Hickerson, Anthony, \& Figura, 2018). These studies are discussed in more detail in the Community Ecology section below.

Physiologically, temperature seems to play a critical role in the efficiency of feeding. Diversity in the gut microbiome of $P$. cinereus begins to decline at $20 \mathrm{degC}$, while digestion efficiency was the greatest at $15 \mathrm{degC}$ (Fontaine, Novarro, \& Kohl, 2018). Two studies have explored the use of stable isotope analysis to study diet 
and other factors (e.g., ecosystem level nutrient cycling; Milanovich \& Maerz, 2012, 2013). These studies are discussed in more detail in the Ecosystem Ecology section below.

\section{(ii) Relative Abundance and Density}

Relative abundance and density were only briefly discussed by Petranka (1998). At that time, several studies had independently suggested that most of the $P$. cinereus population was below the soil surface on any given day. Therefore, Petranka (1998) concluded, counts of salamanders on the surface and less robust mark-recapture methods (e.g., using toe-clips for marking) likely underestimate the true population size. Estimates of density from a variety of locations ranged from 0.21 salamanders $/ \mathrm{m}^{2}$ in Pennsylvania (Klein, 1960) to 2.8 salamanders $/ \mathrm{m}^{2}$ in Virginia (Jaeger, 1980; Mathis, 1991).

Several recent studies that have quantified salamander density in the field. However, one study at the southern range edge in Virginia, indicates that density can be much higher than previous estimates, from 2.49 to 6.26 salamanders $/ \mathrm{m}^{2}$ per plot surveyed over two years (Hernandez-Pacheco et al., 2019; Fig. S2). Most recent papers focus on how salamander abundance is impacted by human activities. These are discussed in more detail in the Conservation Biology and Additional Topics: Management sections below, but here we briefly describe the impact of roads, forest-farm edges, and after prescribed fire on counts of surfaceactive salamanders. Plethodon cinereus occupies forested locations along an urban to rural gradient (Gibbs, 1998b). However, salamander counts are reduced near gravel roads and is likely due to a reduction in soil moisture near roads (Marsh \& Beckman, 2004). Dry conditions at forest-farm edges also lead to reduced counts of salamanders in these ecotones (Young \& Yahner, 2003). An experimental study corroborates these observational patterns, with adults and juveniles found to be more abundant in plots with added moisture and more active in plots with more cover objects (Grover, 1998). Increased abundance of cover objects near to maintained recreational trails may be responsible for increased salamander counts near trails (Fleminget al., 2011). Prescribed fire did not impact capture rates of P. cinereus in Virginia (Keyser et al., 2004) and (Seburn \& Mallon, 2017) found no decline in P. cinereus counts over a 20 year period in Ontario.

\section{(iii) Seasonal Activity and Dispersal}

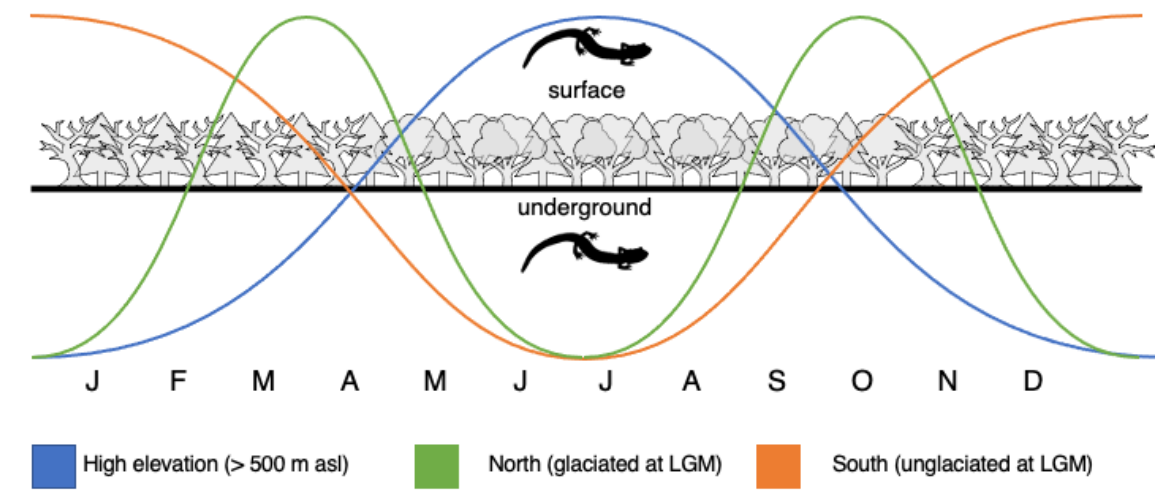

Figure 4. Three regional phenologies of $P$. cinereus surface activity throughout their large range. Biogeographic break points between the three phenologies are approximate based on our experience working with 
this species and four studies (Leclair et al., 2008; Becker et al., 2013; Meshaka \& Wright, 2017; HernándezPacheco et al., 2019). Within a region, there is local variation and we generally find $P$. cinereus to be opportunistic about surface activity during otherwise stressful seasons (winter and summer): if the abiotic conditions (soil moisture and temperature) are suitable, some salamanders will be surface-active. Salamander silhouette from PhyloPic. LGM = last glacial maximum; asl = above sea level.

Petranka (1998) describes how little we know about the activity of salamanders underground and how these patterns vary geographically. Many of the studies cited by Petranka (1998) indicated that there is a seasonal pattern to vertical movement, with suitable soil moisture levels and temperatures driving movement to the surface during the spring and autumn in most locations (north in Fig. 4). One study found $P$. cinereus as deep as $1 \mathrm{~m}$ in winter (Grizzell, 1949). A study by Heatwole (1960) demonstrated that P. cinereus relies on the burrows and tunnels made by other fossorial species to retreat underground, and is likely incapable of doing much more than enlarging existing burrows. Petranka (1998) did not mention any studies on natural dispersal or migration, but did highlight how $P$. cinereus can return to its original location after a $90 \mathrm{~m}$ displacement (Kleeberger \& Werner, 1982).

Based Petranka (1998)'s summary, our own field experience with this species throughout its range, and the four supporting studies described below, we depict the surface activity phenology of $P$. cinereus in Figure 4. There are at least three regional phenologies of surface activity which depend on latitude (north vs. south) and elevation (high vs. low). We hypothesize the latitudinal break between the northern and southern phenologies (Fig. 4) is near the extent of the Wisconsin ice sheet extent at the last glacial maximum (LGM; Fig 1). In support of this hypothesis, Meshaka \& Wright (2017) studied a southern Pennsylvania population of $P$. cinereus approximately $150-200 \mathrm{~km}$ south of the LGM ice edge, and found it to have life history traits more similar to southern populations compared to northern ones (e.g., $75.9 \%$ of females at that site breed annually). Leclair et al. (2008) examines a population near the northern range edge in Québec, showing a slight spring peak and a strong autumn peak in activity. This population also has a notably delayed life history (see above discussion in Breeding and Courtship; Leclair et al., 2008). Finally, both Becker et al. (2013) and Hernández-Pacheco et al. (2019) documented the southern phenology of continuous winter activity (Fig. 4) in Cary, North Carolina and Richmond, Virginia, respectively. Both Petranka (1998) and our experience suggest that $P$. cinereus is opportunistic about surface activity: if temperature and moisture conditions at the surface permit activity, some salamanders will be active, even in winter or summer. There is much to learn on this topic; we do not know how $P$. cinereus determines surface conditions from underground, what the cues are which prompt vertical movement, or even how deep individuals can or will go during stressful seasons.

More recent studies have examined natural and induced movements via displacement and homing to original locations have given some insight into movement patterns of $P$. cinereus. Seasonal movements of adults up a rocky hillside in spring and down in autumn were reported in New York (Woolbright \& Martin, 2014). The authors suggest that this movement is in response to increased availability of crevices in rocks uphill allowing more activity during winter months. A study using drift fences with pitfall traps found that the abundance of salamanders captured was not influenced by proximity to forest edges or streams, but roads likely acted as a barrier to dispersal (Gibbs, 1998a). This is more rigorously supported by Marsh et al. (2005), who found that salamanders displaced across gravel and paved roads have reduced return rates to their original location in comparison to those returning across forest. Similarly, small streams also reduce return rates of salamanders after displacement (Marsh et al., 2007), and both roads and streams limit gene flow, suggesting these landscape features act as dispersal barriers for P. cinereus (Marsh et al., 2007, 2008).

We still know relatively little about natural dispersal distances of $P$. cinereus. Both Petranka (1998) and Williams et al. (1968) speculate that $P$. cinereus is capable of moving great distances, since it presumably expanded from southern refugia to its current geographic extent in southern Canada (Fig. 1) during the last 10-15,000 years after the Wisconsin glacier receded; a distance of at least $1000 \mathrm{~km}$, suggesting dispersal rates are between $67-100 \mathrm{~m}(0.067-0.1 \mathrm{~km})$ per year. Previously mentioned mark-recapture studies (Marsh et 
al., 2004, 2005, 2007) and studies of population genetic structure over relatively short distances in continuous forest or in habitat fragments separated by known dispersal barriers (Cabe et al., 2007; Marsh et al., 2007, 2008; Fisher-Reid et al., 2013; Hantak et al., 2019) suggest that, while individuals generally do not disperse far, they are capable of longer movements across open fields, roads, or streams to return to familiar territory when displaced (25-55 m in Marsh et al., 2004, 2005, 2007) or to colonize new habitat (5-25 m in Marsh et al., 2004). A recent observation increased the maximum distance that a single $P$. cinereus individual is known to have traveled to $143 \mathrm{~m}$ (Sterrett et al. 2015). Dispersing individuals are likely "floaters" - young adults without territories who readily colonize available habitat. Marsh et al. (2004) found that artificial habitat islands installed on an abandoned golf course (i.e., watered areas covered with shade cloth, supplemented with ACOs and leaf litter) were colonized within one year of installation by young adult (based on SVL) $P$. cinereus in Virginia.

The low dispersal needs of individuals likely helps $P$. cinereus persist in small forest fragments where other amphibians with greater dispersal needs have been extirpated (Gibbs, 1998a). It is unclear if P. cinereus engages in sex-biased dispersal. Two studies, both conducted at MLBS in Virginia, either found no evidence of sex-biased dispersal (Cabe et al., 2007) or found evidence females being more philopatric than males, confirmed through lack of genetic structure at short distances in males and greater overall dispersal distances of males (Liebgold, Brodie, \& Cabe, 2011). A more recent study using similar methods for a Maryland population did not find any evidence for sex-biased dispersal (Grant \& Liebgold, 2017). Individual movement behavior is highly repeatable (Cosentino \& Droney, 2016). In unfamiliar environments, individuals from older forest stands moved more than those from younger stands, and edge vs. interior individuals within a stand did not show any movement differences. Individuals showed consistent movement patterns across time, suggesting movement personalities and heritable genetic variation for movement behavior (Cosentino \& Droney, 2016).

\section{(iv) Territories and Home Range Size}

Petranka (1998) devoted several pages to territoriality in both sexes and age classes of P. cinereus. Much of the work he cites and which has been done since, has previously been thoroughly reviewed in Jaeger et al. (2016). To avoid duplication of effort, we focus this section of our review on recent studies that were either (1) independent of the R.G. Jaeger research program, or (2) from non-MLBS locations. Reference information for all studies on this topic returned by our literature search are included in Table S11. We encourage those interested in territoriality and aggression in P. cinereus, which Petranka (1998) notes is a model organism for studying the ecology and evolution of these behaviors, to read (Jaeger et al., 2016). Briefly, there is ample evidence that both males and females defend territory, and that territorial behaviors (e.g., aggression, scent marking, avoidance of residents) vary both seasonally and by local abiotic (temperature, moisture) and biotic (food availability) conditions (Petranka, 1998; Jaeger et al., 2016). At the time Petranka (1998) was published, the average home range area was estimated to be 0.16 to $0.33 \mathrm{~m}^{2}$ with no significant differences between males, females or juveniles. Daily movement of surface-active individuals was estimated at 0.43 $\mathrm{m}$, with increases reported during and after precipitation events (Petranka, 1998). The home range of $P$. cinereus was estimated to be $13 \mathrm{~m}^{2}$ for males and juveniles and $24 \mathrm{~m}^{2}$ for females, and displaced individuals are able to return home at distances of 30-90 m (with declining success at longer distances). Petranka (1998) states that the exact reason $P$. cinereus holds territories is unknown, but it is hypothesized territories are used to defend food, defend or attract mates, and/or defend suitable microhabitats. Petranka (1998) notes that it is believed a large proportion of individuals are floaters that do not hold territories, based on the results of removal experiments (see also Terrestrial Ecology: Seasonal Activity and Dispersal, above). Larger individuals are able to successfully defend their territories from smaller individuals, and males who hold higher quality territories are more likely to attract females. To mark their territories, $P$. cinereus use both fecal pellets and glandular secretions, and they are known to threaten and attack each other during territorial disputes (Petranka, 1998; Jaeger et al., 2016). Interestingly, adult males are less aggressive to juveniles, and they are more tolerant of familiar intruders which suggests the potential for kin recognition and/or a 'dear enemy effect,' in which individuals tolerate near-neighbors to reduce energy expended by acts of aggression 
(Petranka, 1998; Jaeger et al., 2016).

Much of the Jaeger et al. (2016) work on territoriality is lab-based, using MLBS- or Virginia-sourced salamanders. Maerz \& Madison (2000) offer one of the earliest field based studies of territorial behavior and its relationship to food availability. At sites in New York and Pennsylvania with lower food density (as determined by total volume of prey in salamander stomachs), males and females were more territorial, exhibiting higher site fidelity, lower same-sex home area overlap, and body size and foraging success were positively correlated. At sites with higher food density there was lower site fidelity and more same-sex cohabitation of cover among females. Males in the high food environment still defended spaces, likely for female availability (Maerz \& Madison 2000).

Multiple field studies from northern locales have reported aggregations of $P$. cinereus under a single cover object. Quinn \& Graves (1999a) and Placyk, Torretti, \& Graves (2000) document this for populations in Michigan. In Ontario, Rollinson \& Hackett (2015), found modest aggressive behavior in males during the breeding season, but a random distribution of adults during the nonbreeding season. These studies suggest that cooler climates may favor reduced territoriality, since daily foraging activity is generally less restricted than in southern populations. However, this pattern was not found by (Jaeger et al., 2016), who almost exclusively used salamanders sourced from high elevation populations (MLBS and Shenandoah National Park). While the climates of northern latitudes and high elevations are similar, the artificial environment of a laboratory in which most Jaeger et al. (2016) studies occurred may influence the expression of territorial behaviors in P. cinereus. Thus, we are not sure geography alone explains why Michigan and Ontario salamanders appear to be less territorial than high elevation Virginia salamanders. Additionally, one of us (K.L.G.) has seen multiple large aggregations (N [?] 7) under a single coverboard at a low elevation southern site (Richmond, Virginia; Fig. S2), which also has some of the highest reported densities for this species (Hernandez-Pacheco et al., 2019).

Chemical communication is still thought to be very important in territory establishment, identification of individuals, and even determination of potential predation risk. Sullivan, Rohr, \& Madison (2003) found that $P$. cinereus from New York avoid chemical cues from injured salamanders (both conspecific and heterospecific), but do not avoid chemical cues from non-injured salamanders. Earthworms share common vertebrate predators with salamanders, woodland snakes and birds, and thus could be a source of information on general presence of predators, however, salamanders did not respond to chemical cues from either injured or non-injured earthworms (Sullivan et al., 2003). Duhaime-Ross, Martel, \& Laberge (2013) tested both visual and chemical communication in adults and juveniles from Ontario, and found complex interactions between social context, cue type, and age class, suggesting multimodal communication is common. Dawley et al. $(2000,2006)$ studied the cellular physiology of the olfactory and vomeronasal systems across seasons in a Pennsylvania population. Both studies found an increase of cellular proliferation in May and June, and Dawley et al. (2006) found a subsequent increase of apoptotic activity in July, suggesting the importance of these cells during the nonbreeding summer months, when territorial behavior is believed to be strongest (Jaeger et al., 2016).

Site fidelity has been explored in Michigan, Ohio, and Virginia. In Michigan, Placyk \& Graves (2001b) found the same individual under the same cover object one year apart. In Ohio, Martin \& Hantak (2011) document a low level of homing behavior ( $~ 16 \%$ return; similar to reports in Jaeger et al., 2016) in both striped and erythristic individuals displaced $5 \mathrm{~m}$ from their original cover object. Larger individuals are more successful at homing in Virginia (Ousterhout \& Liebgold, 2010). This study also found low levels of between year movements (0.85-1.22 m/year), suggesting high site fidelity (Ousterhout \& Liebgold, 2010). To this we note that we have all seen some level of site fidelity across both seasons and years in our SPARCnet plots in Massachusetts, Virginia, Michigan, Pennsylvania, and New Jersey (M.C.F.R., K.L.G., L.S.M., T.H.J.M. and S.C.S.; unpublished data), but we have not yet quantified these patterns.

Recently, with the advent of visual implant elastomer (VIE) marking methods (Heemeyer, Homyack, \& Haas, 2007), studies have explored field-based space use in P. cinereus. In Virginia, Schieltz, Haywood, \& Marsh (2010) found that adults often use more than one cover object, regardless of spacing between cover objects, 
with males never occupying adjacent cover objects. Munoz et al. (2016b) used spatial capture-recapture (SCR) to understand space use, dispersal patterns, and density in Pennsylvania. This SPARCnet site found densities around 0.423-0.473 salamanders $/ \mathrm{m}^{2}$. Contrary to what is reported in Petranka (1998), Munoz et al. (2016b) found males to use larger areas than females, regardless of season. Dispersal distances between seasons averaged $<1 \mathrm{~m}$, again suggesting high levels of site fidelity (Munoz et al., 2016b). As described above, young adult floaters will quickly take over new habitat when it becomes available (Marsh et al., 2004).

In Ohio, two studies explore territoriality differences between color morphs, and a third studies differences between sympatric $P$. cinereus and $P$. electromorphus. Reiter et al. (2014) found that striped morphs were more aggressive and held on to their territories for longer periods of time than unstriped morph, with both laboratory and field support for these patterns. A follow-up field study by Anthony et al. (2017) found that striped morph territories had $32 \%$ higher quantity of prey than unstriped morph territories. Other work in this population suggests that these differences in territories and territorial behavior between color morphs may play a role in mate choice (e.g., Anthony et al., 2008; Acord et al., 2013). Deitloff et al. (2009) explored the territorial behavior of sympatric and allopatric P. cinereus and P. electromorphus in response to heterospecific intruders in Ohio. Residents did not change behavior based on the source of their intruder, however residents from sympatric populations of both species showed increased aggression toward heterospecific intruders than residents from allopatric populations.

In reviewing the territoriality literature on this species, it is clear that much of what we know stems from a small portion of the very large geographic range; $60 \%$ of the studies we categorized into this topic are from MLBS or other Virginia locations $(\mathrm{N}=21 / 35$; Table S11, Fig. 3). Future work which tests previously documented patterns at other locations is imperative to first, fully understand the social behavior of $P$. cinereus across its range and second, tease apart abiotic and biotic factors which influence the expression of social behavior. We also have much to learn about the details of chemical communication, from the compounds and glands involved to the types of information $P$. cinereus regularly communicates about and with whom.

\section{(v) Miscellaneous Behavior and Sampling Methods}

In the course of our review, we found 24 studies that we initially classified as belonging to the primary category "other," with further comments that these studies related to physiology, behavior, or sensory ecology. Many of these studies were folded into the discussion of their secondary category, and those $(\mathrm{N}=9)$ which remained difficult to place in other sections of the review are summarized here. Generally, these sources focus on behavior that is not directly related to breeding and courtship, foraging, territoriality (all discussed above), or anti-predator tactics (discussed in next section, below).

At the end of Jaeger et al. (2016), the authors note the new work being done on salamander cognition and cognitive ecology. One of the first studies to explore this topic is Uller et al. (2003), which documents rudimentary numerical discrimination in $P$. cinereus. In choice trials containing one, two, three, four, or six food items, salamanders regularly opted for the larger quantity of food in one vs. two and two vs. three forced-choice tests, but could not distinguish between three vs. four or four vs. six. Kight et al. (2005) explored learning in P. cinereus using classical conditioning. Individuals from New Jersey were successfully conditioned to low-frequency sound and vibration, but not to visual or chemical stimuli.

Other studies that were difficult to categorize focused on the physiology of $P$. cinereus. Davis et al. (2009a) found that the roundness of red-blood cells is strongly positively correlated with body condition and body size. The authors suggest that taking blood samples might be a useful non-destructive method of tracking population health over time. Fluorescent powder pigment has been used with success to track amphibian, reptile, and small mammal movement over short durations (e.g., Lemen \& Freeman, 1985; Graeter, Rothermel, \& Gibbons, 2008; Roberts \& Liebgold, 2008), and Orlofske, Grayson, \& Hopkins (2009) was the first study to explicitly test how the fluorescent powders affect respiration and metabolism in a lungless amphib- 
ian using $P$. cinereus. They found that there was no impact on either oxygen consumption or standard metabolic rate (SMR). Instead, it appeared that the stress of being handled was greater than the stress of being temporarily covered in powder (Orlofske et al., 2009). Homyack, Haas, \& Hopkins (2010) found that SMR increased with both body mass and temperature. Novarro et al. (2018) complements this result by assaying corticosterone (CORT) of salamanders living at different temperatures. Salamanders housed at $25 \mathrm{oC}$ had higher CORT levels indicating higher levels of stress, compared to those housed at $15 \mathrm{oC}$. Additionally, salamanders housed at the warmer temperature ate more food than salamanders housed at the cooler temperature (Novarro et al., 2018). Takahashi \& Pauley (2010) observed altitudinal differences in resource allocation. Salamanders from higher elevations (i.e., cooler climates) allocated more energy to storage than growth or reproduction compared to salamanders from lower elevations. Biddle, Grant, \& Liebgold (2017) looked at factors that impact growth, using mark-recapture methods to track individuals over multiple years and seasons. Color morph had no effect on growth, but they did find an effect based on both initial size and environmental fluctuations. Small juvenile salamanders who experienced milder winters showed the greatest growth (Biddle et al., 2017).

The last two behavioral studies that were difficult to categorize both explored the social context of juvenile salamander behavior. Liebgold \& Cabe (2008) explored if juvenile growth rates were affected by familiarity or relatedness of the adults nearby at MLBS. The results varied by year: in the first year, familiarity had a strong effect on mass and size, but not the following year. The authors hypothesize that temperatures may have played a role in the different familiarity results, as the first year was significantly cooler than the second year. Additionally, there was no evidence that familiarity or spatial proximity were indicative of relatedness, or that relatedness to nearby adults affected juvenile growth rates (Liebgold \& Cabe, 2008). Liebgold (2014) followed up on this by testing the presence of territorial adults on juvenile behavior using salamanders from Michigan (presumably non-territorial) and Virginia (presumably territorial; see above). Michigan juveniles were more strongly affected by being housed with territorial adults. In juvenile-juvenile interactions, Michigan juveniles showed increased investigatory and escape behaviors compared to juveniles housed with non-territorial adults.

\section{(g) Predators and Defense}

Petranka (1998) section on predators and defense in P. cinereus was brief, and there has been much work exploring these topics since. Petranka (1998) focused on describing general predators of $P$. cinereus (i.e., birds, snakes, spiders), with an emphasis on snakes, and summarized the experimental studies that have focused primarily on snake predators (garter snakes, Thamnophis spp., and ringneck snakes, Diadophis punctatus ). Petranka (1998) describes the results of one study which suggests that ringneck snakes may be able to distinguish the scent of $P$. cinereus from non-edible prey and even distinguish particular parts of the body (i.e., tail vs. body; Lancaster \& Wise, 1996). In response to a predator, P. cinereus individuals generally change their behavior (i.e., staying motionless or coiling) or autotomize their tails (Petranka, 1998). A measurable proportion of $P$. cinereus populations appears to have broken or regenerating tails, although it is not clear if this indicates predation rates, rates of intraspecific conflict, or, most likely, some combination of both of these. Below, we first discuss new knowledge on the predators and parasites of $P$. cinereus. Next we discuss a handful of studies which have explored differential predation of color phenotypes of $P$. cinereus. Finally, we discuss the many studies which have explored $P$. cinereus defensive behaviors. There are a large number of studies relating to skin and gut microbial communities, particularly how they are involved in defense against the amphibian fungal pathogen $B d$. These have been summarized in their own subsection, the Additional Topics: Microbiota and Disease section below. 


\section{(i) Predators and Parasites}

Since Petranka (1998), a small number of studies have documented unusual predators for $P$. cinereus. Jung et al. (2000) observed a rove beetle (Platydracus vidatus) preying upon live P. cinereus under a cover board in Maryland. The creek chub (Semotilus atromaculatus) was observed preying on $P$. cinereus likely after the salamander was washed into the creek during a flash flood (Sharp et al., 2005). As noted earlier, Hughes et al. (1999) found two salamanders dead inside pitcher plants (Sarracenia purpurea) in bogs in West Virginia and Pennsylvania. These observations are incidental, but there are likely many species that find $P$. cinereus palatable, particularly woodland snakes and ground foraging birds. Most studies of anti-predator responses use chemical cues from $T$. sirtalis fed a diet of $P$. cinereus, other salamander species, or earthworms. These are summarized in the next subsection.

There has been more work done on the parasites of $P$. cinereus, with several studies documenting helminth infections. Muzzall, Peebles, \& Burton (1997) and Bolek \& Coggins (1998) document several species of trematode, nematode, and protozoan parasites on P. cinereus. Most salamanders in Muzzall et al. (1997) hosted at least one parasite, and the trematode (Brachycoelium spp.) was most common in P. cinereus from New Hampshire. In Wisconsin, (Bolek \& Coggins, 1998) found lower overall rates of infection than (Muzzall et al., 1997), but a nematode species (Rhabdias spp.) was the most common parasite on P. cinereus. In Michigan (Gillilland \& Muzzall, 2002) again found high rates of helminth infection, but no evidence that these parasites caused limb deformities. Most studies which have tested wild populations have found low prevalence of the amphibian pathogens Ranavirus or $B d$ on the skin of $P$. cinereus (e.g., Gratwicke et al., 2011; Wunder et al., 2012; Muletz et al., 2012; Augustine \& Neff, 2016), except Jongsma et al. (2019), which found much higher than typical (but still low overall) rates of $B d$ infection in $P$. cinereus from New Brunswick.

Rickettsial intracellular pathogens may also influence $P$. cinereus ecology and health. An rickettsial intraerythrocytic pathogen of the family Anaplasmatacea was found in $16.7 \%$ of 102 P. cinereus individuals tested from Pennsylvania, New York, and Virginia (Davis et al., 2009b). Infected salamanders had significantly larger snout-vent length and more circulating white blood cells than uninfected salamanders. Males in these populations were more likely to be infected than females. The authors hypothesize that the trombiculid mite, Hannemania dunni, a common amphibian ectoparasite is the likely vector, as they are the only ectoparastie which has been observed parasitizing plethodontid salamanders (Davis et al., 2009b). The malebias in infections also suggests a tromiculid mite, as they were previously documented infecting plethodontid salamanders differentially by sex (Anthony, Mendelson, \& Simons, 1994). A follow-up study by (Davis \& Cecala, 2010) focused on Desmognathus ocoee found Rickettsia spp. bacteria, which are obligate intracellular parasites, at a high rate (35\% of $D$. ocoee individuals). This is much higher than infection rates in $P$. cinereus (8\%), and appeared to be a different type of Rickettsia spp. The authors suggest that the difference in prevalence between the two plethodontids is likely due to the difference in their habitat use: $P$. cinereus are completely terrestrial while Ocoee salamanders are semi-aquatic, therefore they contract different species of Rickettsia (Davis \& Cecala, 2010).

There are conflicting reports as to whether or not northern dusky salamanders (D. fuscus) eat P. cinereus. Jaeger, Gabor, \& Wilbur (1998) found evidence to suggest that D. fuscus does eat $P$. cinereus, and that $P$. cinereus actively avoids encounters with $D$. fuscus. However a follow up study by Ransom \& Jaeger (2006) found no evidence of either predation or tail-loss caused by $D$. fuscus, although $P$. cinereus still seemed to avoid $D$. fuscus in the mesocosms. Other large salamanders likely to prey on $P$. cinereus where their ranges overlap include Gyrinophilus porphyriticus and D. quadrimaculatus (Petranka, 1998).

\section{(ii) Differential Predation Risk by Color}

Predation risk for $P$. cinereus may be influenced by color phenotype, although patterns across the range of $P$. cinereus are complex and variable. There is some evidence that the morphs respond differently 
to predators. In Ohio, Venesky \& Anthony (2007) found that striped salamanders spend more time in the 'all-trunk-raised' defensive posture in the presence of neonate T. sirtalis than unstriped salamanders, while unstriped salamanders were more mobile than striped salamanders. A concurrent look at museum specimens showed that unstriped morphs more frequently showed evidence of tail autotomy than striped morphs (Venesky \& Anthony, 2007). These differences in behavior and tail autotomy suggest that the two morphs experience different rates of predation, with the authors hypothesizing that unstriped morphs experience greater predation by T. sirtalis (Venesky \& Anthony, 2007). After simulated predation (i.e., a tail clip), Otaibi, Johnson, \& Cosentino (2017) found that the autotomized tails of striped morphs move longer and faster than those of unstriped morphs.

While differential predation by color is well-documented, which morph gets predated on more seems to depend on the population and the predator (see additional discussion above in Systematics and Geographic Variation). In the range of polymorphic P. ventralis in Tennessee, Fitzpatrick et al. (2009) found that the rare morph was attacked less than the common morph, arguing for negative frequency dependent selection on color phenotype. This was further supported in a Massachusetts population by Kraemer et al. (2016; see above). In Maryland, Grant et al. (2018) found positive frequency dependent selection favoring the more common unstriped morph. Striped morphs were attacked more often by avian predators and had lower survival rates over three years than unstriped morphs. However, there was no observed difference in survival between color phenotypes when mammalian predators were present (Grant et al., 2018). In Pennsylvania, Davis \& Milanovich (2010) observed higher levels of hematological stress markers in unstriped individuals compared to striped, which may be explained by unstriped morphs being exposed to predator attacks more often. In Ohio, Hantak \& Kuchta (2018) found that the striped morph had overall better camouflage, based on reflectance measurements taken in the field, but there was variation in camouflage by season, population, and body region.

Finally, there has been some work exploring the potential Batesian mimicry of the red eft stage of the eastern red-spotted newt (Notophthalmus viridescens) by erythristic P. cinereus (Fig. 1; Kraemer \& Adams, 2014; Kraemer, Serb, \& Adams, 2015a, 2015b). Mimicry by P. cinereus may also be more successful through differences in color, not brightness (Kraemer \& Adams, 2014). Of the predators tested, only birds can determine the difference between newts and striped $P$. cinereus based upon color, not snakes or mammals (Kraemer \& Adams, 2014). Further, newt toxicity does not influence the occurrence of mimicry or the evolution of the mimic phenotype (Kraemer et al., 2015b).

\section{(iii) Defensive Behaviors}

A number of predator-related cues, both simulated and natural, have been documented to alter behavior in $P$. cinereus, and presumably reduce the frequency of predation in nature. In Michigan, Mathis \& Lancaster (1998) demonstrated that exposure to water which previously held stressed conspecifics lead to $P$. cinereus spending more time trying to escape the study chamber relative to individuals exposed to control water. Another Michigan study followed-up on this by showing that $P$. cinereus avoided the skin secretions of stressed conspecifics for up to 2 minutes after the alarm pheromone had been collected (Graves \& Quinn, 2000). In New York, Sullivan \& Jensen (2013) further found that after exposure to different concentrations of damage-released conspecific cues, $P$. cinereus significantly decreased their activity compared to control, but did not discriminate between the differing concentrations of the cue. After simulated predation by tail clips, Roberts \& Liebgold (2008) found that individuals climbed higher than those without simulated predation in the field, especially male individuals.

Most defensive behaviors in $P$. cinereus have been induced using garter snake (Thamnophis spp.), chemical cues (Petranka, 1998; Jaeger et al., 2016). Murray \& Jenkins (1999) tested how P. cinereus responded to cues from T. sirtalis fed different diets in the lab. Using salamanders from Massachusetts, they showed that salamanders avoided the chemical cues of $T$. sirtalis compared to a control, regardless of what prey $(P$. cinereus or earthworms) the snakes had been fed prior to collecting the cue, however, they avoided the $P$. 
cinereus-fed snake cues more strongly than the earthworm-fed snake cues.

Studies which examine salamander responses to garter snake cues have mostly been done using salamanders from upstate New York. A similar pattern of predator avoidance as observed by Murray \& Jenkins (1999) was found by Madison et al. (2002), although the latter study found complex interactions between the time of day, and the combination of cues from snakes fed different diets and stressed or undisturbed conspecifics. Maerz et al. (2001) found that foraging activity decreased with exposure to chemical cues from T. sirtalis. Sullivan, Maerz, \& Madison (2002) found that P. cinereus hid out in refugia when exposed to chemical cues from $T$. sirtalis fed $P$. cinereus in both the lab and field, however time spent in the refugia was significantly shorter in the field than in the lab. This study also speculated that presence of other individual salamanders under a cover object may provide additional protection by keeping salamanders under cover objects when predatory cues are present (Sullivan et al., 2002). A follow up study added that $P$. cinereus also avoided chemical cues from garter snakes fed $E$. bislineata, suggesting that phylogenetically related species display similar antipredator behaviors and can also discriminate against predator diet cues, which may be linked to an overlap in microhabitat (Sullivan, Madison, \& Rohr, 2004). Sullivan, Picard, \& Madison (2005) further describes how the ability of $P$. cinereus to avoid chemical cues of predators is dependent upon both genetic and ecological relatedness of the prey which was consumed.

As noted by Petranka (1998) and in multiple studies discussed above, P. cinereus are known to autotomize their tail in the presence of a threat, and since then, researchers have learned more about the frequency, timing, and effects of this behavior. Wise \& Jaeger (1998) found that in territorial contexts, their own tail loss did not have a large influence on either a resident or intruder's behavior, but they are both more aggressive when facing an opponent without a tail. In the lab, Meche \& Jaege (2002) found that males did not distinguish between tailed or tailless females, however, in the field, they found males associated with tailless females more often. Meche \& Jaeger (2002) offer two hypotheses to explain this result (after rejecting male preference based on their lab results): that females without tails prefer to associate with males, or that male aggression from the courtship process itself results in lost tails for females. Yurewicz \& Wilbur (2004) found that tail loss is energetically costly to females, those with lost or regenerating tails made fewer ova. Meshaka \& Wright (2017) found that males and juveniles had a higher incidence of broken or regrown tails than females in a collection of museum specimens from south central Pennsylvania. Given that Madison et al. (1999) documented the importance of having a tail for proper clutch suspension, it is likely that females are under stronger selection for caution regarding situations that might result in a lost tail than either males or juveniles. (Hessel, Ryerson, \& Whitenack, 2017) found no significant difference in jumping or mobility between individuals with and without tails. Liebgold (2019) documented one case of a female with a bifurcated tail at MLBS in Virginia. Both tail ends had regenerating tips which indicated that autonomy and regrowth can occur in both the main and supernumerary tails. Two of us (T.J.H.M. and K.N.S.) have also seen tail bifurcation in the course of our own field work in Pennsylvania (Figure S3).

\section{(h) Community Ecology}

Petranka's (1998) section on community ecology is limited to $P$. cinereus' interactions with other salamanders, particularly congeners most closely related to it. The section focuses primarily on interactions (or lack thereof) with its sister species, Plethodon shenandoah. According to Petranka, (1998), P. shenandoah and P. cinereus are strongly segregated on the three Virginian mountain tops that $P$. shenandoah occupies, with $P$. shenandoah restricted to the talus habitat, and $P$. cinereus found in every other terrestrial habitat available on the mountains. It was thought that there was coexistence in the talus of Hawksbill Mountain, however, Petranka (1998) recounts Jaeger's (1970) work, which suggests that this is only apparently true. Jaeger (1970) could only find "coexistence" in $P$. shenandoah existing in five talus isolates surrounded by continuous non-talus habitat occupied solely by $P$. cinereus. Jaeger (1970) notes that these are not actually cases of coexistence, as within the isolated talus, there is only P. shenandoah. Petranka (1998) goes on to review what we know about competitive behaviors between these species, and notes that the field and laboratory 
studies are often contradictory, making it difficult to determine what is happening. Petranka (1998) offers Jaeger's (1972) hypothesis that perhaps adult $P$. cinereus exclude juvenile $P$. shenandoah. The remainder of this section of Petranka (1998) briefly discusses three additional interactions $P$. cinereus may (or may not) have with other salamander species: (1) potential for food competition between $P$. cinereus and either $P$. hoffmani or $P$. wehrlei, but lack of support when studied, (2) documented aggressive interactions between $P$. cinereus and both juvenile $P$. glutinosus and adult Desmognathus ochrophaeus, and (3) laboratory predation on $P$. cinereus by Ambystoma maculatum in $9 \%$ of staged encounters.

There has been much additional work on the community ecology of P. cinereus since Petranka (1998), importantly, these studies now regularly include interactions with non-salamander species. We have organized this section to start by reviewing the more recent research on $P$. shenandoah and $P$. cinereus, move on studies on interactions with other salamander species, and then discuss those studies focused on how $P$. cinereus interacts with non-salamander species.

\section{(i) P. shenandoah and $P$. cinereus Interactions}

Recent research has found evidence to further strengthen Jaeger's (1972) argument that $P$. cinereus adults may exclude $P$. shenandoah juveniles. Myers \& Adams (2008) observed phenotypic shifts between allopatric and sympatric populations of $P$. cinereus and $P$. shenandoah but no evidence of character displacement between sympatric populations. This observation suggests competitive exclusion is driving the segregation, restricting $P$. shenandoah to talus, an environment uninhabitable by $P$. cinereus due to its susceptibility to desiccation (Petranka, 1998; Myers \& Adams, 2008). An early genetics study on P. cinereus and $P$. shenandoah found no evidence of introgression or hybridization (Carpenter et al., 2001), however rare hybrids have been more recently documented using both genetics and morphology (E.H.C. Grant, pers. comm.), suggesting a more complex dynamic exists between the two species than previously thought.

Additional investigation into competitive interactions between the species found that, under current climate conditions, $P$. shenandoah lost more mass during competition than $P$. cinereus, indicating $P$. cinereus is competitively superior to $P$. shenandoah (Dallalio, Brand, \& Grant, 2017). Predicted future climate warming conditions suggest a more costly outcome for $P$. cinereus though, as average mass loss was comparable for the two species under presumed future (warmer) temperatures. However, during intraspecific trials, $P$. cinereus experienced no difference in mass loss between current and future conditions. This suggests $P$. cinereus has a larger range of temperature tolerance than $P$. shenandoah (Dallalio et al., 2017). Finally, Chambert et al. (2018) found that while $P$. cinereus populations were relatively stable over four years, $P$. shenandoah experienced high turnover in occupancy.

\section{(ii) Other salamanders and $P$. cinereus Interactions}

Regarding competition with salamanders other than P. shenandoah, Petranka's (1998) comments are limited. At that time, research indicated that interspecific competition for food between $P$. cinereus and $P$. hoffmani is unlikely and that dietary overlap between $P$. cinereus and $P$. wehrlei occurs but competition for food is unknown (Petranka, 1998). Petranka (1998) also briefly describes interactions of $P$. cinereus with two more distantly related salamanders. Adult $P$. cinereus display similar levels of aggression towards and defend territories against juvenile $P$. glutinosus as they would towards adult conspecifics. Desmognathus ochrophaeus defends cover objects from $P$. cinereus and drives $P$. cinereus out of occupied territories (Petranka, 1998).

Studies since Petranka (1998) have further described interactions between $P$. cinereus and other closely related salamander species. Multiple studies have examined one-on-one interactions between $P$. cinereus and other small-bodied Plethodon, looking for evidence for character displacement or competitive exclusion. Character displacement in both head morphology and diet is supported for $P$. cinereus and $P$. hoffmani (Adams, 2000; Adams \& Rohlf, 2000), but not for teeth number or jaw muscle mass (Swart \& Adams, 
2004). While the microhabitats between these two species are not significantly different (Carlson, Thawley, \& Graham, 2016), Jaeger et al. (2002) found behavioral differences that may drive character displacement: $P$. cinereus is more aggressive and less submissive than both allopatric and sympatric $P$. hoffmani. They suggest that the boundary between the two species is either static, due to the aggressive superiority of $P$. cinereus and the exploitative superiority of $P$. hoffmani from eating larger prey, or that $P$. cinereus is slowly intruding on the range of $P$. hoffmani (Jaeger et al., 2002).

Character displacement has also been supported for sympatric P. cinereus and P. nettingi (Adams, West, \& Collyer, 2007; Adams, 2011). Both species are smaller when in sympatry than when in allopatry, and sympatric populations show greater divergence in head shape between species relative to allopatric populations. These results suggest interspecific competition as a mechanism for phenotypic variation among Plethodon species (Adams et al., 2007). Over 32 years, P. nettingi has decreased in abundance, which Kroschel et al. (2014) found may be correlated to $P$. cinereus expanding their territory into the higher elevations that $P$. nettingi originally inhabited, but they could not rule out changes in environmental conditions affecting both species.

The ranges of $P$. cinereus and $P$. electromorphus overlap, however sympatric localities are not common, suggesting competitive interactions are important (Deitloff et al., 2008). In intraspecific trials, P. cinereus was more aggressive than $P$. electromorphus, and in interspecific trials, $P$. electromophus was more submissive. These results suggest $P$. electromorphus may not be able to expand its range into that of $P$. cinereus, but $P$. cinereus may invade the range of $P$. electromorphus (Deitloff et al., 2008). In sympatric populations, both $P$. cinereus and $P$. electromorphus exhibited more aggressive behaviors and less submissive behaviors than allopatric populations (Deitloff et al., 2009). This study, however, only looked at one sympatric and one allopatric population, and the authors called for future research over the full extent of the overlapping ranges. Site-specific morphological differences also occur in sympatric $P$. cinereus and $P$. electromorphus populations (Deitloff, Petersen, \& Adams, 2013). At some sympatric locations the two species experienced morphological divergence in head shape, while at other sympatric locations, morphological convergence occurred. These results suggest that abiotic and biotic disparities at different locations may be responsible for the morphological variation (Deitloff et al., 2013). Similarly, in Kentucky and Ohio, P. cinereus overlaps with $P$. richmondi, however sympatry is uncommon, and there seem to be climatic differences which correspond to differences in distribution (Hedeen, 2000). Plethodon richmondi was found in drier, upslopes compared, while P. cinereus was found in wetter, lower, flatter areas (Hedeen, 2000).

The ranges of $P$. cinereus and $P$. hubrichti are parapatric, with the range of $P$. cinereus completely surrounding that of $P$. hubrichti. Aasen \& Reichenbach (2004) found no significant encroachment of $P$. cinereus on P. hubrichti. Arif et al. (2007) found P. hubrichti to be more aggressive and less submissive than $P$. cinereus, but no differences in diet. Head shape between these two species was different, but there was no evidence of ongoing character displacement in sympatric populations (Arif et al., 2007). The authors suggest $P$. hubrichti restricts the range of $P$. cinereus, even though it lives in suitable microclimates for $P$. cinereus. This was further supported by Kniowski \& Reichenbach (2009) which found that sympatric $P$. hubrichti and $P$. cinereus were similar in microhabitat preferences, movement patterns, growth rates, and adult and juvenile survival rates but differed in size. Sympatric $P$. hubrichti neonates and adults were larger than $P$. cinereus neonates and adults (Kniowski \& Reichenbach, 2009). A follow-up study observed non-fatal biting by a larger $P$. hubrichti of a smaller $P$. cinereus, suggesting interference competition contributes to the geographic distribution of these species (Kniowski \& Pietsch, 2015).

A number of studies explored the position of $P$. cinereus within the larger woodland salamander community, focusing on its interactions with stream specialists (e.g., Desmognathus spp. and Eurycea spp.) and largebodied Plethodon like P. glutinosus. As described above in the Predators and Parasites section, it is unclear if D. fuscus preys on P. cinereus (Jaeger et al., 1998; Ransom \& Jaeger, 2006). Jaeger et al. (1998) additionally found no evidence that larger $P$. glutinosus eat smaller $P$. cinereus but a later study finds that when sympatric, P. glutionsus and P. cinereus reduce each others' growth (Price \& Secki Shields, 2002). Two other studies support exclusion of $P$. cinereus by Desmognathus spp. Grover (2000) found little 
habitat overlap between Plethodon and Desmognathus, but some between Plethodon and Eurycea. Grover \& Wilbur (2002) found that D. fuscus displaced P. cinereus closer to streams, and both abundance and mass of $P$. cinereus increased with distance from streams. When limited to a near-stream habitat, the body condition of $P$. cinereus individuals declines over a three week period, where stream specialist E. cirrigera likely outcompetes it (Pasachnik \& Ruthig, 2004). A removal experiment at a stream/forest ecotone found that removal of $P$. cinereus had no effect on either P. cinereus abundance or Desmognathus sp. abundance, however E. cirrigera moved closer to streams when P. cinereus was removed (Ransom \& Jaeger, 2008).

Plethodon salamanders are hypothesized to use behavioral mechanisms, such as climbing behaviors, that promote coexistence. A field study conducted by Mezebish, Blackman, \& Novarro (2018) observed the climbing frequencies of three size guilds of Plethodon species. The authors found that the smallest species, $P$. cinereus, climbed more frequently than both the intermediate species, $P$. montanus, and the largest species, $P$. glutinosus. Plethodon cinereus also increased climbing frequency when exposed to intraspecific competitors but decreased climbing when exposed to interspecific competitors. The abundance of intraspecific competitors also resulted in an increase in climbing behavior in P. cinereus (Mezebish et al., 2018). The differences in climbing behaviors in different sized Plethodon suggest that behavioral niche partitioning may be a mechanism that reduces competitive interactions and allows for the coexistence of species. In the lab, while adult $P$. glutinosus and $P$. cinereus did not directly interact when placed together, $P$. cinereus increased nose tapping behaviors when in the presence of $P$. glutinosus (Mezebish et al., 2018). This suggests that the individuals were chemically aware of the presence of the other. This study also found that more climbing behavior was observed in all species when there was more understory vegetation available to climb on.

(iii) Non-salamander Species and $P$. cinereus Interactions

Several studies since Petranka (1998) have described interactions of $P$. cinereus with non-salamander species, largely focusing on relationships with intraguild predators common to the forest floor and soil (e.g., spiders, centipedes, beetles, earthworms). The majority of recent work, conducted at MLBS in Virginia, focuses on the relationships between native and non-native earthworms (Annelida) and P. cinereus. Caceres-Charneco \& Ransom (2010) found that P. cinereus uses earthworm burrows as refuge. In both lab and field, they found that $P$. cinereus was more likely to be under cover objects when earthworms were absent, and more likely to be in burrows when earthworms were present (Caceres-Charneco \& Ransom, 2010). Ransom (2017) also observed that salamanders do not seem to distinguish between native vs. non-native earthworms, both consuming and using the burrows of all species similarly. Ransom (2012) defines three distinct relationships between earthworms and $P$. cinereus : (1) earthworms are ecosystem engineers, building burrows that salamanders use regularly; (2) earthworms are potential prey for salamanders; and (3) earthworms compete with other microinvertebrate prey species leading to indirect effects on salamanders. Ransom (2012) finds that the strength of these relationships varies by season for the non-native earthworm Lumbricus terrestris. Positive effects of ecosystem engineering were important to overwinter survival of adult $P$. cinereus. The positive effect of earthworms as prey was only greater than the negative effect of earthworms as competitors in the summer (Ransom, 2012).

In Michigan, a survey by Brunges et al. (2020) investigated the effect of 11 different invasive earthworm species on terrestrial salamander abundance. They found a negative association between salamander counts and the presence of invasive, epigeic (i.e., found in the leaf litter) earthworms but no effect of invasive endogeic (i.e., found underground) earthworms. The authors suggest that this is because epigeic earthworms consume the O-horizon leaf litter layer, which $P$. cinereus depends on for moisture and protection (Brunges et al., 2020). Similarly, in Virginia, Ransom (2017) found that the presence of the invasive earthworm, Octolasion tyrtaeum, had an indirect, negative effect on nighttime $P$. cinereus counts. This earthworm species was also associated with a narrower O-horizon layer suggesting a similar mechanism to that proposed by (Brunges et al., 2020). Finally, two studies in Ohio found that non-native Amynthas earthworms alter microhabitats and behavior of $P$. cinereus. The first found that earthworm presence decreased leaf litter mass over time, leading salamanders to increase movement, cover object use, and co-occurrence under cover objects with earthworms 
(Ziemba et al., 2015). A follow-up study found further evidence the salamanders were negatively impacted by Amynthas and Metaphire spp. (Ziemba, Hickerson, \& Anthony, 2016). In the lab, salamanders opted for poorer quality microhabitats and consumed fewer flies when earthworms were present. In the field, salamanders and earthworms shared cover objects much less than expected, and earthworm abundance was negatively associated with juvenile and male salamander abundance (Ziemba et al., 2016).

In Ohio, several studies have explored relationships between $P$. cinereus and spiders, centipedes, and carabid beetles. Anthony, Hickerson, \& Venesky (2007) found that P. cinereus exhibited more submissive behaviors when exposed to native centipedes as opposed to introduced centipedes, suggesting the presence of learned predator avoidance behaviors in P. cinereus. Burgett \& Smith (2012) exposed P. cinereus to centipedes or conspecifics, and found salamanders showed increased aggression towards centipedes regardless of their residency status but showed no increase of aggression towards conspecifics in either setting. Hickerson et al. (2012) did a removal experiment with P. cinereus- and centipede-removal treatments. Interactions were complex: removing either predator changed the abundance of other invertebrate predators, and had a mix of effects on invertebrate prey species. There was a negative relationship between spider or centipede abundance and $P$. cinereus abundance, suggesting interference competition between salamanders and the two invertebrate predators (Hickerson et al. 2012). However there was a positive relationship between P. cinereus and carabid beetles. Hickerson et al. (2012) note that these relationships are strongest during the dry summer months, when these species all have fixed territories tied to suitable microhabitats. Interestingly, in the lab, Gall, Anthony, \& Wicknick (2003) find a negative relationship between carabid beetles and $P$. cinereus. Residents of both species behaved more aggressively than intruders, and attacked intruders of both species at similar levels. Hickerson et al. (2018) found that spiders in the genus Wadotes are perceived as competitors by $P$. cinereus in laboratory trials, and there was no evidence of intraguild predation between spiders and salamanders.

Several studies explore the role of $P$. cinereus in regulating the composition of soil-litter invertebrate communities and ecosystem processes. These interactions are thought to be crucially important for forest health, and often are used to justify the importance of woodland salamanders more generally (e.g., Welsh \& Droege, 2001; Davic \& Welsh, 2004). In reviewing the literature on this topic, we find many sources do support the important role of $P$. cinereus in forest ecosystems, however, at least two studies found no direct effects of $P$. cinereus on a variety of community and ecosystem processes (Homyack et al., 2010; Hocking \& Babbitt, 2014). Here, we focus on the community level impacts of $P$. cinereus, and summarize ecosystem level impacts in the Additional Topics: Ecosystem Ecology below.

In Ohio, both Walton (2005) and Walton \& Steckler (2005) found that $P$. cinereus does affect the composition of soil-litter invertebrate communities, but these effects vary seasonally. When leaf litter mass and moisture were both high, salamanders exerted stronger top-down effects on mesofaunal invertebrates (Walton, 2005). In microcosm experiments, when P. cinereus was present, Walton \& Steckler (2005) found more podomorphic Collembola, fewer enchytraeid worms, and fewer macroinvertebrate detritivores. Total invertebrate density, and densities of Collembola and Acari (mites) increased more quickly when salamanders were present. The authors argue that salamanders are either preying on larger detritivores that compete with these mesofaunal groups, releasing them from competition, or by being present, salamander fecal matter promotes microflora growth. A follow-up study found that salamander treatments again increased the density of Collembola, but this time only in urban leaf litter, not rural leaf litter (Walton, Tsatiris, \& Rivera-Sostre, 2006). Other taxa experienced decreased densities in both urban and rural leaf litters when salamanders were present: oribatid mites, pseudoscorpions, isopods, and millipedes (Walton et al., 2006). In contrast, in Virginia, Homyack et al. (2010) found no evidence that $P$. cinereus affected the number of invertebrate taxonomic groups or functional guilds. Hocking \& Babbitt (2014) found similar results in New Hampshire. In their study, $P$. cinereus had no effect on intraguild predator abundance (carabid beetles, centipedes, and spiders). We feel these conflicting results are to be expected, given the large geographic range and diversity of forest types that $P$. cinereus finds itself in, however, they make generalizations about the role of plethodontid salamanders in these communities and ecosystems difficult, and caution is warranted using $P$. cinereus as an ecological indicator within a given forest until local patterns are sufficiently understood. 
Finally, a small number of studies have looked at the indirect relationships between $P$. cinereus and other woodland species, including white-tailed deer (Odocoileus virginianus), garlic mustard (Alliaria petiolata ), and Lymantria dispar dispar (LDD) moths, and fungal communities. In Ohio, Greenwald, Petit, \& Waite (2008) found a positive, indirect effect of deer presence on $P$. cinereus abundance. Salamanders were three times more abundant in deer-permitted control plots compared to deer-exclusion plots. Greenwald et al. (2008) suggest that deer herbivory increases invertebrate species richness through increased nutrient deposition and plant growth, which in turn attracts salamanders. In Massachusetts, Brooks (1999) found that the abundance of $P$. cinereus did not change across forests with higher or lower densities of white-tailed deer. In Ohio, removal of invasive garlic mustard had no effect on P. cinereus abundance (Smith, 2018). Two studies, one based in Ohio (Walker et al., 2015) and the other in West Virginia (Walker et al., 2018), used a metagenomics approach to explore the impact of the removal of $P$. cinereus on the fungal detritivore food web. Fungal community diversity and composition were not affected by removal of $P$. cinereus, but there were significant shifts in relative abundance of the fungal groups (Walker et al., 2014, 2018). Mitchell (2015) explored how $P$. cinereus counts changed before and after invasion by LDD moths. Despite recovery of the canopy and increased amounts of coarse woody debris, (Mitchell, 2015) observed a $>60 \%$ decline in $P$. cinereus counts in 1993 compared to 1988 (prior to the 1989 arrival of LDD moths). It is not clear if the decline in $P$. cinereus was related to the arrival of LDD moths, other factors, or a combination of these.

\section{(i) Conservation Biology}

Petranka's (1998) account of the conservation biology of $P$. cinereus was very brief, focusing on sensitivity of $P$. cinereus to soil acidification (e.g., Wyman, 1988) and intensive forest harvesting practices (e.g., deMaynadier \& Hunter, 1995). Due to the ubiquitous ecology of $P$. cinereus, there was very little concern for the impacts of human-mediated effects on $P$. cinereus populations due in part to the then growing understanding that $P$. cinereus are remarkably abundant in some forests; with biomass greater than other prominent vertebrate groups (e.g., New Hampshire; Burton \& Likens, 1975a). Like many of the topics we reviewed thus far, the conclusions of conservation-focused studies of $P$. cinereus are geographically variable, with many studies finding contradictory patterns, suggesting the importance of understanding both landuse history and current anthropogenic threats to best determine the likelihood of persistence for any given population. We found many recent papers which have looked at how $P$. cinereus responds to specific kinds of anthropogenic change, as well as many studies that focus on how to manage $P$. cinereus populations or use $P$. cinereus to manage forest ecosystems. We have separated the more management-focused sources to be summarized under the Additional Topics: Management header below, and here focus largely on concerns related to how $P$. cinereus responds to anthropogenic land use changes and climate change. We also limited our discussion of papers which have conservation as a secondary category (Table S11), as these studies are nearly all discussed elsewhere in our review.

Since Petranka (1998), many studies have further explored the sensitivity of $P$. cinereus to anthropogenic influences, despite low prioritization of this species as a conservation concern. deMaynadier \& Hunter (1998) found that $P$. cinereus in Maine are sensitive to clear cutting and edge effects, and that $P$. cinereus have an affinity for mature forest. Further, it was suggested that modified, open canopy sites may represent sink habitat, meaning there are significantly more juveniles than adults and these juveniles may not have established territories. Despite this finding, Brooks (2001) found that P. cinereus abundance only decreases temporarily, both when eastern hemlock is partially harvested as well as when wooly adelgid invasions causes hemlock defoliation and tree loss. Within three years of initial tree loss, the abundance of $P$. cinereus had already begun to increase (Brooks, 2001). Mossman et al. (2019) found that abundance of $P$. cinereus were the highest in 20-25 year-old shelterwoods and significantly higher than in mature or unharvested forest sites, suggesting $P$. cinereus may benefit from some infrequent level of disturbance, particularly disturbance that results in increased availability of coarse woody debris, like timber harvesting. This contrasts with the findings of Rodewald \& Yahner (1999) in Pennsylvania, who found that harvested areas had no salamanders, and the abundance of $P$. cinereus in mature forest was positively correlated with the number of trees. 
Knapp et al. (2003) compared declines in salamander abundance due to clear-cutting to six other silvicultural methods, and found that if the canopy was disturbed, the other silvicultural methods were as bad as clearcutting for salamander abundance. After a forest fire in Nova Scotia, only one P. cinereus individual was found alive about 300 meters from the edge of the wildfire in an unburned refuge (Russell, 2011). No salamanders were found in the burned area when searched over the next four months. This suggests that $P$. cinereus may be negatively affected by wildfires in terms of habitat loss; however, given their ability to persist in subterranean retreats during a wildfire, they likely have the ability to move to areas nearby that were not affected by the wildfire once it is safe to do so (Russell, 2011).

Various modeling approaches by Leroux et al. (2017) suggest that patch size is more important for persistence of $P$. cinereus populations than either patch isolation or environmental conditions (but see discussion of conservation genetics below). A model by Venier et al. (2007) based on P. cinereus in Ontario concluded that $P$. cinereus are sensitive to changes in habitat availability, regardless of whether the disturbance is caused naturally or from intensive harvesting. As canopy cover and downed coarse woody debris increases, $P$. cinereus abundance increases (Venier et al., 2007). In Indiana, however, while Peele et al. (2017) also found these factors to be positively related to the abundance of $P$. cinereus, neither one had a significant effect on the survival rates of the P. cinereus. Otto, Roloff, \& Thames (2014) found similar importance of canopy cover and coarse woody debris availability in Michigan at the landscape scale, but not at the local scale. A model by Gordon et al. (2012) found that P. cinereus abundance decreases following forest harvesting in Ontario. Vanderwel et al. (2011) modeled the value of different habitats following deforestation, using $P$. cinereus data from Quebec and Vermont. The habitat value for $P$. cinereus increased after most stages of deforestation, likely due to increases in coarse woody debris, suggesting that $P$. cinereus are capable of recovering after disturbances to their environment. A field study in West Virginia by Wood \& Williams (2013) found that reclaimed mountaintop removal mines are not suitable habitat for P. cinereus, as they favor more of a grassland or shrubland habitat than forest. They argue that the recovery of the forest habitat after mountaintop mining may take longer than areas that are clear-cut due to factors like soil compaction.

In Virginia, D.M. Marsh and colleagues have published a number of studies on the effects of roads on $P$. cinereus, many of which we have previously discussed above (Marsh \& Beckman, 2004; Marsh et al., 2005, 2007, 2008). From a conservation perspective, both (Marsh \& Beckman, 2004) and (Marsh et al., 2007) examine the edge effects of roads. The first study found strong edge effects - fewer $P$. cinereus were found close to roads in all three populations studied. The authors suggest that the decline of $P$. cinereus as you approach a road edge is due to the parallel decline in both soil moisture and cover object area near roads (Marsh \& Beckman, 2004). Marsh (2007) focused on gated (closed to vehicles) vs. ungated roads and found no edge effects on $P$. cinereus from roads that are narrow and gated, while ungated roads show the same pattern as observed in (Marsh \& Beckman, 2004). Marsh et al. (2005, 2008) both find that roads are dispersal barriers, using a displacement and homing experiment (Marsh et al., 2005) and measures of population genetic structure (Marsh et al., 2008). A similar study by (Gibbs, 1998a) in Connecticut also found roads to be a dispersal barrier for $P$. cinereus.

As mentioned earlier, several studies have explored the relationship between habitat fragmentation and population genetics. Gibbs (1998c) compared continuous to fragmented populations in Connecticut and found that the fragmented populations were more divergent (showed greater genetic structure) compared to the continuous populations. Gibbs (1998c) also noted a difference in morph frequency, fragmented populations were close to 50:50 striped:unstriped, while continuous populations were nearly 100\% striped. In Quebec, Noel et al. (2007) found that urban P. cinereus have both lower allelic richness and lower heterozygosity. A follow up study found that $P$. cinereus show more genetic differentiation in areas where anthropogenic disturbances have persisted for a longer time (Noel \& Lapointe, 2010). The authors suggest that this is because $P$. cinereus in these environments tend to have a low effective population size. Further implications from Noel \& Lapointe (2010) are that it is important to preserve large, forested areas within cities in order to increase genetic variation and conserve or increase effective population size. Cosentino \& Brubaker (2018) demonstrate the importance of connectivity for post-agricultural recovery. Salamanders were affected by historical land use, and were more abundant in areas of historical forest cover. This suggests connec- 
tivity between relic source populations and regenerating forests on post-agricultural lands is important for colonization of the new forest habitat (Cosentino \& Brubaker, 2018).

Using a combination of contemporary field measurements of SVL and historical measurements from museum specimens, Caruso et al. (2014) found that the body size of P. cinereus has decreased by $7 \%$ in several Appalachian mountain locations over 55 years. The greatest decreases in body size was in the southern Appalachians, which have had the greatest drying and warming. The authors hypothesize that this correlation between location and body size likely comes from an increase in metabolic expenditure (Caruso et al., 2014). In contrast, using only museum specimens but including specimens from both the Appalachian mountains and coastal plain, McCarthy et al. (2017) reported a similar precipitation pattern, with body size being negatively correlated precipitation in the driest month, but the opposite temperature pattern: larger $P$. cinereus were correlated with higher mean maximum July temperatures, and specimens on the coastal plain showed a temporal increase in body size correlated with a temporal increase in mean maximum July temperatures. Specifically in response to Caruso et al. (2014), and prior to the publication of McCarthy et al. (2017), both Grant (2015) and Connette, Crawford, \& Peterman (2015) offer alternative explanations for shrinking salamanders. They argue that Caruso et al.'s (2014) results could be explained by the sampling approaches used in the study and that both museum specimens and contemporary surface-active individuals likely only represent a portion of the inferred population. Further work on this topic which tracks populations across the range over time using robust spatial capture-recapture methods (e.g., Sutherland et al., 2016) should be able to more confidently detect these patterns.

\section{(j) Additional Topics}

In addition to the miscellaneous physiology and behavior sources we report on above, we found at least four additional topics not covered by Petranka (1998), which we feel there has been enough literature to warrant their own sections. First, we present studies on the ecosystem ecology of $P$. cinereus, a topic we found strangely absent from Petranka (1998). Next, we summarize studies which focus on the natural resources management of $P$. cinereus and the forests in which they live. Third, we describe the many studies exploring the skin and gut microbiota of $P$. cinereus and their relationship to disease resistance. Finally, we found several ecotoxicology studies which explore the tolerance of $P$. cinereus to various anthropogenic pollutants common to current and former United States military installations.

\section{(i) Ecosystem Ecology}

There are surprisingly no references to the role of $P$. cinereus in ecosystem level processes in Petranka's (1998) otherwise thorough species account. Petranka (1998) mentions Burton \& Likens' (1975a) study at Hubbard Brook Experimental Forest in New Hampshire for their estimates of P. cinereus density, but Petranka (1998) does not cite or mention Burton \& Likens (1975b), a simultaneous study on the energy flow and nutrient cycling that must pass through $P$. cinereus, given their position in the trophic web and the high densities observed at Hubbard Brook. Neither study is restricted to P. cinereus, however, P. cinereus far outweighs the estimated biomass contributed by the other salamander species (Burton \& Likens, 1975a, 1975b). The pair of Burton \& Likens' (1975a, 1975b) studies have since been cited by hundreds of subsequent studies, some of which argue for the use of $P$. cinereus as an ecosystem indicator species (e.g. Welsh \& Droege, 2001; Siddig, Ochs, \& Ellison, 2019), or for the overall importance of plethodontids to terrestrial forest ecosystems (e.g., Davic \& Welsh, 2004), and indeed have shaped the thinking of many researchers working on North American plethodontid salamanders since (but see Riedel et al., 2008 and Moore \& Ouellet, 2015 for caution about the use of $P$. cinereus as an indicator species). Thus, we found it odd that ecosystem ecology was neglected by Petranka (1998), although this seems to be the case for all species accounts throughout the book. 
Since Petranka (1998), several studies have investigated how P. cinereus contribute to ecosystem processes like nutrient cycling or energy movement. Some of these have manipulated densities of $P$. cinereus or examined how natural density variation influences ecosystem processes; albeit with contradictory findings (Wyman, 1998; Walton, 2005; Sucre et al., 2010; Homyack et al., 2010; Walker et al., 2014; Hocking \& Babbitt, 2014; Pelini et al., 2015; Hickerson, Anthony, \& Walton, 2017). In New York, Wyman (1998) found that experimental cages with two or six salamanders (compared to controls with zero salamanders) significantly decreased forest floor prey communities, which had an indirect negative effect on leaf litter decomposition, likely due to $P$. cinereus feeding on leaf shredders. In Ohio, Hickerson et al. (2017) also manipulated $P$. cinereus densities by removal and found comparable treatment effects in reducing leaf litter decomposition but also on microbial communities. In contrast, other experimental studies found no effects of $P$. cinereus on ecosystem measures, such as leaf litter and wood decomposition or nitrogen mineralization rates, among others,even when manipulated densities were as high as 4 salamanders $/ \mathrm{m}^{2}$ (Homyack et al., 2010; Hocking \& Babbitt, 2014). Additionally, (Sucre et al., 2010) found that salamander abundance was not a reliable indicator of nitrogen availability.

Milanovich \& Maerz $(2012,2013)$ describe the use of stable isotopes from P. cinereus tail clips for tracking ecosystem nutrient and energy movement. They note that these isotopes are accurate even when the salamander has been fasted for up to 35 days, making their interpretation much easier compared to organisms for which fasting does change these values. (Gilbert et al., 2014) use carbon-13 to understand how carbon from sugar maple (Acer saccharum) tree roots moves through the ecosystem, and found that $P$. cinereus individuals contained large quantities of root-derived carbon. This was true whether earthworms were part of the treatment conditions or not (Gilbert et al., 2014).

Another line of investigation has made connections between ecosystem changes or elemental gradients to $P$. cinereus populations or energetics (Homyack, Haas, \& Hopkins, 2011; Beier et al., 2012; Ochs \& Siddig, 2017). In recently harvested forests in West Virginia, $P$. cinereus expends more energy on body maintenance, although there were no consistent patterns related to body condition or invertebrate abundance (Homyack et al., 2011). In New York, Beier et al. (2012) found a positive relationship between $P$. cinereus abundance and a well-defined calcium gradient, while in Massachusetts, Ochs \& Siddig (2017) found P. cinereus was more prevalent in forests with lower soil $\mathrm{pH}$, lower carbon to nitrogen $(\mathrm{C}: \mathrm{N})$ ratios, and lower temperatures. The authors note that the first two characteristics, low soil $\mathrm{pH}$ and low $\mathrm{C}: \mathrm{N}$ ratios, are characteristic of hemlock stands, which used to be much more common within the range of $P$. cinereus prior to the arrival of hemlock wooly adelgid (Siddig et al., 2019). The loss of hemlocks and other massive ecosystem changes as a result of invasive species who affect fundamental environmental properties relied on by forest floor inhabitants (e.g. soil chemistry, temperature), may also influence P. cinereus (e.g., Caceres-Charneco \& Ransom, 2010; Ochs \& Siddig, 2017). Continental invasions of earthworms undoubtedly influence $P$. cinereus in many ways, but especially by changing soil properties and $P$. cinereus behavior, which likely influence the role of salamanders in shaping North American forest ecosystems (Caceres-Charneco \& Ransom, 2010; see also Community Ecology section above).

Lastly, as discussed in Community Ecology above, P. cinereus' role on the forest floor as a top predator (e.g., Walton, 2005; Walker et al., 2014) may influence heterotrophic respiration rates, having measurable implications for climate change in forest soils, but these effects may vary latitudinally (Pelini et al., 2015). The inconsistent results of these studies (see discussion above and in Community Ecology) are yet another reason it is critical to design robust experiments to better understand variation in demography and community interactions across the range of $P$. cinereus (see section below on SPARCnet).

\section{(ii) Management}

In Petranka (1998) there was relatively little consideration of management for $P$. cinereus populations or their habitat, presumably because $P$. cinereus is considered to be a common species throughout its range, despite its proposed sensitivity to changing landscapes driven by human activity and our knowledge of these 
practices on other woodland salamanders. However, since Petranka (1998), there has been a great deal of research on the impacts of forest management on amphibian communities, including the ubiquitous $P$. cinereus. Here, we summarize those studies which focus on managing forests with explicit mention of impacts to $P$. cinereus.

In an experimental management context (i.e. either manipulated or observational), $P$. cinereus abundance or distribution may be influenced by factors as diverse as prescribed fire (Mitchell, 2000), tree harvest management strategy or intensity (Messere \& Ducey, 1998; Rodewald \& Yahner, 1999; Harpole \& Haas, 1999; Yahner, Bramble, \& Byrnes, 2001a; Duguay \& Wood, 2002; Perkins \& Hunter, 2006; Patrick, Hunter, \& Calhoun, 2006; Homyack \& Haas, 2009; Strojny \& Hunter, 2010b; Hocking, Babbitt, \& Yamasaki, 2013; MacNeil \& Williams, 2014; Mossman et al., 2019), deer management (Greenwald et al., 2008; see discussion in Community Ecology above), managed grazing (Riedel et al., 2008), or subsequently in forests with varying densities and qualities of coarse woody debris (McKenny et al., 2006; Strojny \& Hunter, 2010a). There is some evidence that plantation-style forests, common on lumber farms, are not suitable for $P$. cinereus (Waldick, Freedman, \& Wassersug, 1999; Reams et al., 2008) despite the closed canopy, perhaps due to lack of cover objects.

Studying how timber harvest practices influence P. cinereus energetic response, Homyack et al. (2011) reported on short-term effects of forest floor temperatures from overstory removal harvest, which may lead to an energetic cost for $P$. cinereus. The authors also suggest that retaining downed logs and other large woody debris in addition to promoting regrowth of understory may contribute to rapid recovery of forest floor conditions conducive to stable P. cinereus populations. Electric transmission right-of-way (ROW) areas are often heavily managed to remove vegetation and to provide continual access for maintenance. Yahner et al. (2001a) report that $P$. cinereus were among the most common amphibians found in these areas and that ROW areas housed a greater diversity of amphibians than adjacent forests; however, the authors do not discuss how detection influenced their results. In a follow-up study, $P$. cinereus were more commonly found in areas in Pennsylvania that are managed through timber harvesting than in areas that are undisturbed (Yahner, Piergallini, \& Ross, 2001b), which contradicts (deMaynadier \& Hunter, 1998)'s finding that $P$. cinereus prefer interior, mature forests. (Yahner et al.'s (2001b) work does fit with Riedel et al.'s (2008) suggestion that $P$. cinereus may be less sensitive to anthropogenic change than previously thought, provided they have appropriate microhabitats (i.e., cool and moist cover objects; but see Wood \& Williams, 2013 for a counter example).

Some studies demonstrated no effect of experimental management on $P$. cinereus individuals or populations. Mahoney et al. (2016) showed no effect of shelterwood-burn or fenced shelterwood-burn treatments on $P$. cinereus abundance, but stressed that leaf litter and fine cover objects were influential on $P$. cinereus abundance when considering best approximating single variable models. Similarly, Moore (2014) used a mesocosm experiment to demonstrate that liming, or the addition of lime to acidic, maple forests, has no impact on survival of P. cinereus. Cameron, Hickerson, \& Anthony (2016) found similar results in the field comparing a long-term liming site to an unlimed control area in Ohio.

In the last two decades, it has been suggested that $P$. cinereus be considered as an indicator species for a variety of ecosystem properties (e.g., Welsh \& Ollivier, 1998; Welsh \& Droege, 2001). Siddig et al. (2019) agreed that $P$. cinereus may be an indicator species used to monitor changes in eastern hemlock forest ecosystems due to their sensitivity to key stressors and changing environmental conditions. Along with several studies hypothesizing and testing the connection between a changing climate and color phenotype ratios in a population (see Systematics and Geographic Variation, above), Moore \& Ouellet (2015) concluded that climate variables do not influence color phenotype ratios broadly across the range of $P$. cinereus. The indicator species concept (also known as "surrogate species concept") arose as a shortcut approach to evaluating the complex state of ecosystems by measuring a few, relatively inexpensive observable characteristics (Landres, Verner, \& Thomas, 1988; Caro, 2010; Lindenmayer, Barton, \& Pierson, 2015). The degree to which $P$. cinereus can successfully be considered an indicator species in the future will depend upon making direct connections between $P$. cinereus and specific drivers of interest as well as understanding the mecha- 
nistic relationship between the indicator and ecosystem property; often an unobservable ecological process (Fleming et al., 2020).

\section{(iii) Microbiota}

Information regarding the microbiota living on the skin of $P$. cinereus prior to 1998 was scarce, partly due to the early stages of the necessary technology for microbial community profiling available at that time. Petranka (1998) mentions that there has been no evidence for antibiotic properties in the skin secretions of brooding females, a claim which has since been refuted by several recent studies which document microbial taxa which produce both antibiotic and antifungal compounds in clinically relevant levels (e.g., Fredericks \& Dankert, 2000; Harris et al., 2006; Culp, Falkinham, \& Belden, 2007; Lauer et al., 2007; Brucker et al., 2008a, 2008b; Becker et al., 2009). Most research to date regarding the skin microbiota of $P$. cinereus has focused on two overarching goals: (1) to identify microbial taxa found on salamander skin and in their guts, and (2) to understand the potential these microbial taxa have for the production of antibacterial and antifungal compounds. The latter goal has been specifically focused on those microbiota which may be useful in targeting the deadly amphibian fungal pathogen, Batrachochytrium dendrobatidis (hereafter, $B d$ ), to which $P$. cinereus is thought to be largely resistant to.

Fredericks \& Dankert (2000) is one of the earliest studies to identify antimicrobial proteins on the skin of $P$. cinereus, shortly after Petranka (1998) was published. However, as this study focused on protein fractions instead of whole-community DNA, they are unable to determine if these compounds are salamander-made or microbe-made. Harris et al. (2006) document three genera of bacteria on the skin of $P$. cinereus that inhibit the growth of $B d$ in challenge assays (Bacillus; Lysobacter; Pseudomonas). Another early study of skin microbes by (Culp et al., 2007) isolated four bacteria (Pseudomonas fluorescens; Microbacterium testaceum, Flavobacterium johnsoniae, Bacillus cereus, and Corynebacterium spp.) and one yeast (Candida molishiana ), as well as several unidentifiable isolates. Of these, Pseudomonas fluorescens was especially notable since it is known for having antimicrobial and toxic (hydrogen cyanide) properties. Culp et al. (2007) also noted that many of the bacterial colonies were mucoid, and thus are likely involved in preventing salamander skin dehydration.

Shortly after these early studies, metagenomic methods became less expensive and more accessible, and most subsequent work using these methods suggests that microbes produce many of the beneficial proteins previously identified by Fredericks \& Dankert (2000). Lauer et al. (2007) found that $89 \%$ of the salamanders in their sample had strong and/or weak antifungal bacteria on their skin. They used a fungal pathogen, Mariannaea sp., isolated from dead Hemidactylium scutatum eggs in their challenge assays. Lauer et al. (2007) highlight their discovery of a specific bacterial strain closely related to Janthinobacterium lividum, a bacteria which was previously known to produce antifungal compounds. In their study, J. lividum was found on every salamander (Lauer et al., 2007). This result has since been confirmed by several additional studies which focus on $J$. lividum and $P$. cinereus 's ability to either resist or quickly recover from $B d$ infection (e.g., (Brucker et al., 2008b; Becker et al., 2009; Wiggins et al., 2011; Muletz et al., 2012; Hess et al., 2015). A follow-up study found similar microbial composition at the genus and family levels, but little overlap at the species level, on the skin of confamilial H. scutatum (Lauer et al., 2008).

Janthinobacterium lividum makes at least two known antifungal metabolites, indole-3-carboxaldehyde and violacein, that are lethal to $B d$ in naturally occurring concentrations found on $P$. cinereus 's skin (Brucker et $a l ., 2008 \mathrm{~b}$ ). When $B d$ is experimentally introduced salamanders, eight out of ten salamanders who died due to $B d$ infection had no violacein-producing microbes and the remaining two had lower than average violacein levels (Becker et al., 2009). Other Bd inhibiting or mitigating microbial taxa found in the skin or in the guts of $P$. cinereus include Lysobacter gummosus (Brucker et al., 2008a), Pseudomonas reactans (Harris et al., 2006), and several taxa identified by (Muletz-Wolz et al., 2017) and (Fontaine et al., 2018).

In a lab experiment, Loudon et al. (2014a) cultured bacterial isolates including Bacillus spp., J. lividum, Pseu- 
domonas spp., and Chitinophaga arvensicola, from P. cinereus as co-cultures and mono-cultures to determine what bacterial species or combinations most successfully inhibit the growth of $B d$. Metabolites in the co-cultures were most inhibitory to $B d$ as they interact synergistically and additively (Loudon et al., 2014a). Pseudomonas sp. was found to prevent weight loss observed in Bd treatments by (Harris et al., 2006). There is some evidence that these protective taxa can be inoculated on salamanders who lack them (Muletz et al., 2012; Loudon et al., 2014b). Muletz et al. (2012) sought to use J. lividum to protect the globally declining amphibian populations as a result of $B d$, and learned that through soil bioaugmentation, it is possible to experimentally introduce $J$. lividum into soil which is then environmentally transferred to amphibian skin. Within five days post- $B d$ exposure, the environmental transmission of $J$. lividum was able to inhibit the colonization of $\mathrm{Bd}$ on the skin of P. cinereus (Muletz et al., 2012). Loudon et al. (2014b) identified a "core community" of eight operational taxonomic units (OTUs) which was found on at least $90 \%$ of the salamanders throughout the experiment. Their experiment revealed that the most abundant and prevalent OTU was a member of the phylum Verrucomicrobia, and that five out of the eight core OTUs were in the family Pseudomonadaceae which all have antifungal properties. Their results also indicate that the availability of different bacteria in the environment influences what bacteria are found on the salamander's skin. Specifically, they suggest that the environment works as a reservoir for bacteria. Without access to this reservoir, the core community of skin microbiota dominates and the diversity of the microbiome is lower. Loudon et al. (2016) supports these results: nine out of 12 (75\%) OTUs that were over-represented on $P$. cinereus skin had at least a $97 \%$ match to antifungal isolates. They also noted that four of these overrepresented OTUs were part of the core community identified by Loudon et al. (2014b). Loudon et al. (2016) suggests that salamander skin, as an environment, may actively select for bacteria with antifungal activity. Future efforts to protect amphibians from mortality due to diseases such as $B d$ may include exposing captive animals to environmental reservoirs with defensive bacteria. This may improve captive-rearing programs for amphibians to prevent colonization of Bd (e.g., Passos, Garcia, \& Young, 2018).

Protective bacteria for $P$. cinereus against fungal diseases may also originate from the gut of the salamander, although we only found two studies that looked at gut microbes (Wiggins et al., 2011; Fontaine et al., 2018). Wiggins et al. (2011) suggest that the gastrointestinal tract of $P$. cinereus could serve as a reservoir for $J$. lividum. Fontaine et al. (2018) also find evidence of Janthinobacterium and other disease resistance related taxa in the guts of $P$. cinereus. This study found an interesting pattern with temperature. Microbial diversity, including those taxa involved in disease resistance, declined at $20 \mathrm{oC}$ compared to $10 \mathrm{oC}$ and $15 \mathrm{oC}$, and digestive performance was greatest at $15 \mathrm{oC}$. Additionally, the relative abundance of pathogenetic taxa increased at 20oC (Fontaine et al., 2018).

In terms of how $P$. cinereus responds to $B d$ infection, there have been a small number of studies which document behavioral effects of $B d$ infection, differential infection by color phenotype, and gene expression. As noted earlier in the Predators and Parasites section, natural incidence of $B d$ infection in $P$. cinereus is generally quite low ([?] 2\%; with the exception of a New Brunswick population whose infection rates are around 13\%; Jongsma et al., 2019). In a behavioral study, $B d$-infected salamanders attacked prey significantly more often than non-infected individuals and the prevalence of $B d$ among $P$. cinereus decreased over time (Hess et al., 2015). The authors suggest that $P$. cinereus may have natural resistance to $B d$ (Hess et al., 2015). Venesky et al. (2015) looked at differential effects of $B d$ infection by color phenotype in the laboratory. Unstriped $P$. cinereus had both a higher prevalence of infection and higher mortality due to $B d$ than the striped individuals (Venesky et al., 2015). The authors note that these results conflict with a previously noted connection between high levels of melanin and disease resistance, but that the behavioral differences between morphs (e.g., Venesky \& Anthony, 2007; Davis \& Milanovich, 2010; Reiter et al., 2014) may increase the stress experienced by unstriped morphs thus reducing their resistance to infection. Finally, Ellison et al. (2020) found changes in gene expression, mediated by temperature, in P. cinereus who were uninfected, recently infected, or naturally recovered from infection with $B d$. At lower temperatures, inflammation transcripts were more commonly expressed, while at higher temperatures, salamanders shift to increased expression of adaptive immune genes, including MHC (major histocompatibility complex) genes (Ellison et al., 2020). 
We only found one study which looks at how presence or absence of $P$. cinereus may impact soil microbial communities, and the results are somewhat equivocal (Hickerson et al., 2017). While removal of intraguild predators had little effect on soil and leaf litter microbial communities, the treatment in which salamanders were most abundant (centipedes removed) suppressed bacteria which in turn decreased the rate of leaf litter decomposition (Hickerson et al., 2017). Additionally, only a small number of studies explore change in microbial communities over space and time (Muletz-Wolz et al., 2017, 2018; Muletz-Wolz, Fleischer, \& Lips, 2019; Barnes, Carter, \& Lewis, 2020). The work of C.R. Muletz-Wolz and colleagues has explored skin microbial communities across an elevational gradient in Maryland and Virginia. They found that location, rather than host characteristics, was the best predictor of prevalence of antifungal bacteria (Muletz-Wolz et al., 2017); higher elevations had greater alpha diversity of bacterial taxa than lower elevations (MuletzWolz et al., 2018); and increasing the pathogen load and increasing temperatures changed the microbial community structure, which suggests that $P$. cinereus may not be resistant to $B d$ in all ecological contexts (Muletz-Wolz et al., 2019). Finally, in New York, Barnes et al. (2020) found that the composition of bacterial communities, but not overall diversity, varied with a land use gradient (urban, suburban, exurban), but there were similar levels of $B d$ inhibition among each of these different land use bacterial communities.

Studying the interactions of $P$. cinereus with skin, gut, and environmental microbes is still a relatively new research focus. We also note that this topic is one of the most geographically restricted, with $71 \%$ $(17 / 24)$ of studies discussed above using salamanders from Virginia, and the remaining seven studies using salamanders from adjacent central, mid-Atlantic states (Ohio, West Virginia, Pennsylvania, Maryland, and New York). There is evidence that microbial taxa change over time, space, and with access to soil reservoirs (e.g., Loudon et al., 2014b; Muletz-Wolz et al., 2018; Barnes et al., 2020), thus standardized microbial sampling, such as that described by (Walker et al., 2015), is needed throughout the range of $P$. cinereus to fully understand its relationships with microbial taxa.

Other gaps in salamander microbiota research include exploring the distribution of different bacteria across the body regions of the salamander. Culp et al. (2007) suggests there may be a non-random distribution of different species in different body regions and that future work should look to swab the whole animal at multiple locations on the body to determine if/where different species may congregate. As we noted above, this field is lacking in geographical diversity, despite multiple studies finding local differences in microbial taxa (e.g., Muletz-Wolz et al. 2017; Barnes et al., 2020). We also do not fully understand the relative roles and interactions of microbial metabolites versus salamander skin secretions. Lauer et al. (2007) suggests separating and analyzing the antibiotic chemicals produced by the animal from those produced by bacteria on the skin. They also suggest conducting experiments using antibiotics to determine the roles of different cutaneous bacteria.

\section{(iv) Ecotoxicology}

The final additional topic that has grown since Petranka (1998) explores the responses of P. cinereus to a variety of anthropogenic contaminants. These ecotoxicology papers generally subject individual salamanders to multiple concentrations of compounds with known toxicity in other vertebrates. In most cases, the concentrations tested are well below field values at contaminated sites, and salamanders show some ability to detect and avoid some of these contaminants.

Several studies using salamanders from Massachusetts and Maryland have focused on contaminants commonly found on current and former United States military installations, including RDX (1,3,5trinitrohexahydro-1,3,5-triazine; Johnson et al., 2004); 2,4-DNT (dinitrotoluene; Johnson, Suski, \& Bazar, 2007); TNT (trinitrotoluene; Bazar et al., 2008); copper (Bazar et al., 2009); lead (Bazar et al., 2010); and HMX (high melting explosive or octahydro-1,3,5,7-tetranitro-1,3,5,7-tetrazocine; Johnson et al., 2010). All of these except HMX had negative effects on $P$. cinereus at the highest concentrations tested (Johnson et al., 2004, 2007, 2010; Bazar et al., 2008, 2009, 2010). The most common symptoms were weight loss, depressed white blood cell counts, and death. Additionally, in the copper treatments, the authors observed 
lethargy, unresponsiveness, and inability to right oneself (Bazar et al., 2009). HMX did not seem to affect $P$. cinereus, and the authors suggest that this could be because of differences in how HMX is absorbed through salamander skin versus through the gastrointestinal tract of the other vertebrates tested (Johnson et al., 2010).

Mercury $(\mathrm{Hg})$ concentrations found in $P$. cinereus increases when exposed to contaminated soil when compared to individuals from non-contaminated sites (Bergeron et al., 2010a). Bergeron et al. (2010a) reported higher $[\mathrm{Hg}]$ in $P$. cinereus than any previous literature, but also noted a low sample size of 24 individuals. It is not clear from this study if $\mathrm{Hg}$ accumulation has negative effects on the salamanders or if it should be more of a concern for the predators that eat salamanders. A follow-up study found that total $\mathrm{Hg}$ and (mono)methylmercury (MMHg) concentrations are positively correlated in P. cinereus from contaminated sites (Bergeron et al., 2010b). The same study did not find accumulation of selenium (Se) above what is expected for normal background. Selenium is thought to be antagonistic with Hg, offering some protection from $\mathrm{Hg}$ toxicity (Bergeron et al., 2010b). Due to P. cinereus's capacity to take in $\mathrm{Hg}$ from the surrounding environment, it is suggested that $P$. cinereus be used as a bioindicator to monitor $\mathrm{Hg}$ accumulation in the landscape (Townsend \& Driscoll, 2013).

Two laboratory studies have demonstrated that $P$. cinereus is able to detect and avoid contaminated soils, suggesting that contaminated sites in nature would simply not be colonized by salamanders. First, Gertzog et al. (2011) found that $P$. cinereus can detect and avoid substrates which have been contaminated with herbicides such as Roundup, Bayer Brushkiller, and Spectracide Brush Killer. Second, Gaglione et al. (2011) found that $P$. cinereus can detect and avoid substrates contaminated with urea. Finally, a third study tested the effects of the microbial pesticide Bacillus thuringiensis var. kurstaki (Btk) on P. cinereus abundance or diet in West Virginia, and found no adverse effects (Raimondo, Pauley, \& Butler, 2003).

\section{Is Plethodon cinereus an EEB Model Organism?}

In completing the comprehensive review, it is clear that Duffy et al.'s (2021) requirement that a model system have been studied long enough to generate a "substantive body of knowledge" (pg. 55) has been met by the research to date on P. cinereus. In defining EEB model systems, Duffy et al. (2021) highlight Travis' (2006) related stipulations that "robust inference requires horizontal comparisons and vertical integration" (p. 307). These, too, have been clearly met by the research to date on $P$. cinereus, which has been used to better understand closely related, but more geographically restricted, taxa (horizontal comparisons) and has been studied at nearly every scale of biological organization (vertical integration). Outside of those who work on plethodontid salamanders, however, we do not think the EEB community would necessarily designate $P$. cinereus as a model organism; not in the comprehensive way that others readily provide examples such as Anolis lizards, Mimulus plants, or Drosophila flies (e.g., see Table S1 from Duffy et al., 2021).

Thus, P. cinereus falls short of model organism status. This is due to many of the challenges around developing new model systems laid out in Duffy et al. (2021). In particular, the broader appeal of $P$. cinereus research and plethodontids as model organisms more generally has been hampered by a lack of infrastructure-building, historical gatekeeping, funding constraints, and other limitations imposed on both academic and non-academic scientists in the United States and Canada. Since P. cinereus is not a model organism yet, we have the rare opportunity to build the infrastructure required of successful model systems around $P$. cinereus and other plethodontid salamanders with a deliberate eye towards inclusive, coordinated collaboration and knowledge sharing. In this section, we first describe how although the EEB community clearly values data collected at both long temporal scales and large spatial scales for illuminating fundamental phenomena (Fig. 2; Hughes et al., 2017), it can be challenging and risky for an individual scientist to structure their research program around such data generation (Duffy et al., 2021); yet such structured approaches are essential for data quality control and quality assurance (Clutton-Brock \& Sheldon, 2010). We then describe and recommend coordinated distributed experiments (CDEs; Fraser et al., 2013) as a model experimental 
design for generating data sets of long temporal and large spatial scales. Finally, we demonstrate how we have put a CDE into practice for $P$. cinereus with our collaborative research network, The Salamander Population and Adaptation Research Collaboration Network (SPARCnet). We hope that our experience provides a blueprint for other researchers to more confidently develop the diversity of model systems and scientists called for by Duffy et al. (2021).

\section{(1) Go big or go home: Long-term studies over large spatial scales}

The intellectual pursuits of EEB fields are deeply intertwined (Fig. 2), such that the fundamental phenomena which model systems are tasked with illuminating are often overlapping and difficult to isolate in both practice and theory (see, for example, Sutherland et al., 2013). Illuminating these fundamental phenomena in EEB not only benefits from use of diverse model systems (Duffy et al., 2021; Wale \& Duffy, 2021), but also from long-term, individual-based data sets (Clutton-Brock \& Sheldon, 2010), as many of the fundamental phenomena are best viewed from the perspective of tracking many individuals over long periods of time. Much like the perpetual discussions in the literature about what counts as a biomolecular model organism, there has been much discussion in EEB on what counts as long-term data, how best to initiate and maintain a long-term study, and the disproportionate value of long-term data sets for both basic research and applied policy, as evidenced by number of publications and citation indices (e.g., Clutton-Brock \& Sheldon, 2010; Hughes et al., 2017). In parallel to these discussions, globalization has proceeded at a rapid pace with high speed internet connections and smart devices invading our homes, workspaces and field sites, making possible large collaborative research networks which take advantage of the relative ease of modern communication to coordinate large scale experiments across time and space. Fraser et al. (2013) term these collaborative research efforts CDEs, and note their particular value to testing large spatial-scale hypotheses in EEB fields.

CDEs are, in part, an answer to the difficulties of synthesizing data across studies with disparate experimental designs, as is traditionally done in meta-analyses (Fraser et al., 2013) or with museum specimens (Grant, 2015). Beginning in the late 20th century with the Long-Term Ecological Research (LTER) network (Hobbie et al., 2003), there has been a steady proliferation of CDEs in the early decades of the 21st century. While coming up with an exhaustive list of these CDEs is beyond the scope of our paper, we note for example, that the faculty authors of this paper belong to EREN (The Ecological Research as Education Network; Gartner et al., 2020), Snapshot USA (Cove et al., 2021), and SPARCnet (Sutherland et al., 2016; Munoz et al., 2016b; Hernandez-Pacheco et al., 2019), and we have used SquirrelNet (Dizney et al., 2020), LTER, and NEON (National Ecological Observatory Network; Keller et al., 2008) data in both our teaching and research.

CDEs like those listed above could be to EEB what databases and networks like FlyBase (Drysdale, Crosby, \& The FlyBase Consortium, 2005) and The Jackson Laboratory (Dewsbury, 2012) are to the biomolecular and biomedical sciences: the infrastructure and community ethos needed to successfully develop a new EEB model system by using standard, simple methods across research groups, time, and space. Leonelli \& Ankeny (2013) argue that both infrastructure and the community ethos are critical to the development and success of a model organism, and this argument is echoed by both Duffy et al. (2021) and Wale \& Duffy (2021) for $\mathrm{EEB}$, the latter of which explores models within the subfield of the ecology and evolution of infectious disease. Many of the 25 EEB model systems listed in Duffy et al.'s (2021) supplement have substantial infrastructure and a thriving research community, including established field sites and protocols (e.g., Barro Colorado Island Forest Census Plot; Kress et al., 2009) and community-building events like subgroup meetings in tandem with national conferences (e.g., Mimulus meetings; Mimubase.org, 2021).

Fraser et al. (2013) and Borer et al. (2014) both argue that one important quality of successful CDEs is that they are not prohibitively expensive or prohibitively time consuming for participants. The majority of the authors on this paper are faculty and students at primarily undergraduate institutions (PUIs), and our ability to do research is more time and budget restricted than our peers at research-intensive (R1) institutions. Many of the CDEs we know of and participate in have both education and research as central 
missions. In our experience, CDEs make it easier for PUI faculty and students to collaborate with R1 faculty and students and thus aid PUI faculty in maintaining active research programs while also teaching high contact-hour loads. This is achieved in part because CDEs make use of the per capita staffing potential of a PUIs' undergraduate classrooms. CDEs further help to include contingent, non-tenure track faculty and their students at both types of institutions in research activities. Simultaneous with the proliferation of CDEs over the last two decades, there has been a pedagogical pivot towards developing course-embedded undergraduate research experiences (CUREs) as a means to provide authentic research experiences to more students than faculty at PUIs and R1s alike could possibly individually mentor (e.g., Shortlidge, Bangera, \& Brownell, 2017). This focus of most CDEs on both research and education is perhaps best embodied in EREN's mission statement (ERENweb.org, 2021):

"To create a model for collaborative ecological research that generates high-quality, publishable data involving undergraduate students and faculty at primarily undergraduate institutions (PUIs)."

The dual education and research missions of many CDEs also appeals to United States-based funding agencies, like the National Science Foundation (NSF), which scores proposals not just on intellectual merit, but also on broader impacts. By having education explicitly baked into the research proposal through CUREs and PUI collaborators, CDEs can make a broader impact argument for their proposed work. This is important because, as Clutton-Brock \& Sheldon (2010) argue, the biggest challenge facing long-term studies in EEB is maintaining consistent funding. Hughes et al. (2017) also note this challenge, although the pair of papers disagree on what counts as a long-term study. Clutton-Brock \& Sheldon (2010) use a minimum of 10 years, while Hughes et al. (2017) use a minimum of four years to define long-term. Hughes et al. (2017) directly connect their minimum to academic funding cycles: NSF typically funds short-term (four years or less) and long-term ( $>$ four years) proposals, and four years is the typical length of a graduate student's Ph.D. project, excluding coursework. The authors further demonstrate that the proportion of research dollars going toward short-term studies has steadily increased in the 21st century, while the proportion of research dollars going towards long-term studies steadily decreased, despite the disproportionate value placed on long-term studies (see their Fig. 2; Hughes et al., 2017). This speaks in part to what Duffy et al. (2021) note as the one of the challenges to EEB model system development: that neither early career scientists nor funding agencies are willing to shoulder the risks inherent to starting a long-term or large spatial-scale study. To this we also note that there is a funding disconnect between NSF's short-term funding, long-term funding, and the minimums discussed by both Clutton-Brock \& Sheldon (2010) and Hughes et al. (2017). NSF's Long Term Research in Environmental Biology program (LTREB; National Science Foundation, 2021) requires six years of continuous data at the full proposal stage in order to be eligible to apply for five years of funding with the potential to renew for an additional five years pending favorable review. This means a long-term study in the eyes of Clutton-Brock \& Sheldon (2010; > 10 years) requires two consecutive short-term NSF grants in order to simply qualify for a LTREB that could cover the following 10 years.

\section{(2) A Nascent Infrastructure for Plethodon salamanders in SPARCnet: The Salamander Population and Adaptation Research Collaboration Network}

SPARCnet was founded in 2013 by researchers at the United States Geological Survey Amphibian and Reptile Monitoring Initiative (USGS ARMI) and Pennsylvania State University (SPARCnet.org, 2021). Since then, it has grown to include over 30 research collaborators and 13 education collaborators (e.g., nature centers) across 13 states and provinces within the geographic range of $P$. cinereus (Fig. S4). SPARCnet methodology employs a simple and inexpensive, yet robust and spatially explicit mark-recapture design (Sutherland et al., 2016; Munoz et al., 2016b; Fleming et al., 2020, 2021) using standardized ACO plots, arranged in pairs for potential experimental and control studies (e.g., snow removal, forest management). Despite the challenges of long-term individual based studies (i.e., consistency, quality, stable funding; Clutton-Brock \& Sheldon, 2010), SPARCnet holds annual meetings and has developed research protocols and minimum core data collection agreements among researchers that allow this network to meet the growing needs of long- 
term studies focusing on changing demography of the focal species, P. cinereus (Clutton-Brock \& Sheldon, 2010). SPARCnet's framework is flexible, allowing individual researchers to design side projects that are site-specific or network-wide (i.e., collaborating on data collection with other research plots), which may focus on questions and topics as diverse as those reviewed in this paper. Additionally, the flexibility of SPARCnet structure allows for researchers to exploit observations and research questions that emerge in real time (i.e., emergence of disease or toxicology) and work together to rapidly study these phenomena across the geographic range in tandem.

CDEs generally, and SPARCnet in particular, provide a solution for consistent data collection methods and helps network participants address the many inconsistencies that come with studying a wide-ranging species like $P$. cinereus. It is well known that it is difficult to study patterns from past data based on differences in methodology and confounding factors from data collection (Fraser et al., 2013; Grant, 2015), and that EEB studies typically suffer from geographic and taxonomic biases, as we have demonstrated throughout the review above (Fig. 3). A geographically- and intellectually-distributed network, such as SPARCnet, addresses these concerns to provide a robust spatial and temporal assessment of the current state and change over time of $P$. cinereus populations across its range, as well as a unique educational framework for engaging students at all levels and community members. As we have highlighted in our review, there is much geographic variation in $P$. cinereus biology: puzzling aspects of $P$. cinereus physiology, behavior, roles in ecosystems, and potential roles as an indicator species (especially among color phenotypes; see Systematics and Geographic Variation, above). Some have criticized these inconsistencies (e.g., Moore \& Ouellet, 2015), however in light of Duffy et al. (2021) and in the hindsight of this review, we think the inconsistencies, the natural variation of a single species over a wide geographic area, is exactly what makes $P$. cinereus an ideal EEB model organism. SPARCnet is poised to address many of these inconsistencies (1) with existing standardized data collection, (2) minimal protocol changes disseminated throughout the network, and (3) with more substantial protocol pivots among a subset of willing network participants.

Unlike the other seven of the top eight most well-studied salamander species, $P$. cinereus is the only member of the Plethodontidae (Table 4; Table S2). As a direct-developing member of the largest family of salamanders, $P$. cinereus is also more representative of the majority of ecologically and evolutionarily successful salamander taxa than any of the other top seven species. What we learn about being a salamander by studying $P$. cinereus is more likely to apply to vulnerable and endangered plethodontids than what we learn by studying $A$. maculatum or $N$. viridescens. Combined with their large population sizes and position in the forest food web, we see endless potential for illuminating fundamental phenomena in EEB with $P$. cinereus. On top of the overwhelming volume of ecological and behavioral knowledge we have summarized above, there is also a very healthy level of systematic interest in both Plethodontidae and the genus Plethodon (e.g., Wiens et al., 2006; Fisher-Reid \& Wiens, 2011; Fisher-Reid, Kozak, \& Wiens, 2012), making plethodontids truly a model clade, not just $P$. cinereus a model organism.

In terms of the three axes Duffy et al. (2021) include in their Figure 1, we know, and demonstrate with our review, that $P$. cinereus is both highly lab tractable and field tractable, and research can be and has been done at all biological scales aside from global (Fig. 5). The one Achilles heel of $P$. cinereus as a model organism which we could come up with is that controlled laboratory breeding has not yet been possible (hence the lower vertical dimension representing lab tractability in Fig. 5). But females can be induced to lay fertilized eggs (e.g., Evans et al., 2020), and courtship behaviors can be observed (Jaeger et al., 2016), so perhaps one day we will crack the code for controlled breeding of $P$. cinereus, but, as we argue above, this requires infrastructure, community ethos, and investment from funding agencies. 


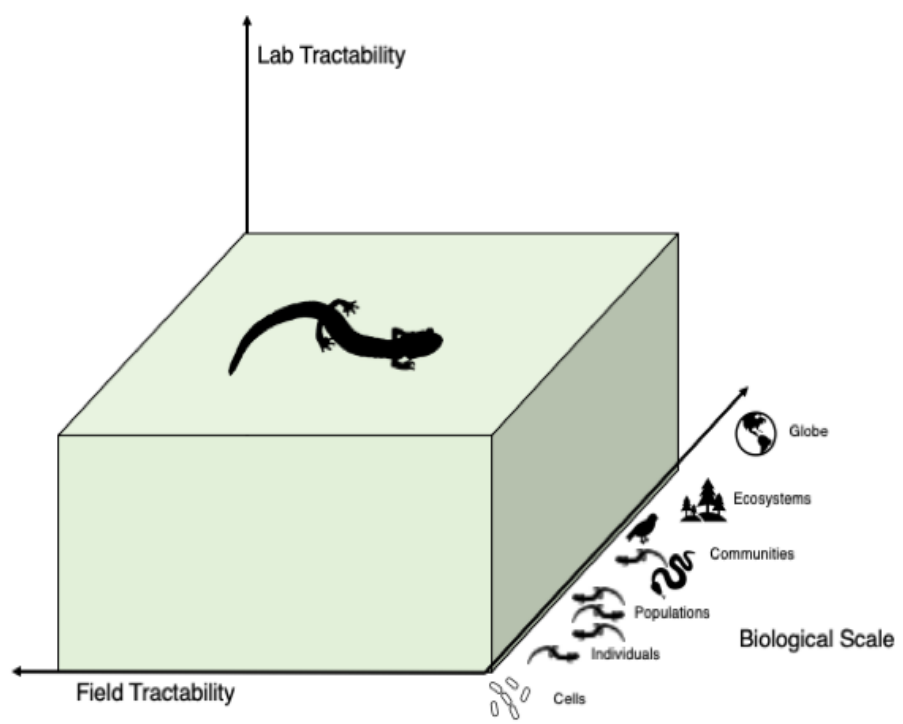

Figure 5. The versatility of $P$. cinereus as a model organism in EEB. Salamanders are highly tractable in both lab and field, and at all biological scales through ecosystems. Lab tractability is lower than field tractability because a protocol for controlled breeding in captivity has not been successfully developed yet. Figure modeled off of Figure 1 from Duffy et al. (2021).

\section{Conclusions}

1. Model systems in evolution, ecology, and behavior (EEB) are similar, but also different from those in biomolecular and biomedical sciences. Variation is a feature, not a bug.

2. As lungless ectotherms, salamanders in the family Plethodontidae have great potential to serve as a model clade within EEB.

3. One plethodontid in particular stands out, P. cinereus, for the overwhelming depth of knowledge accumulated in the last several decades, its critical niche position in forest ecosystems, its accessibility and tractability in both lab and field, and its close, well-studied relationships with other members of its family.

4. In just 22 years, over 400 papers on all aspects of $P$. cinereus' evolution, ecology, and behavior have been published in peer-reviewed journals. While studies are highly localized and demonstrate high levels of local variation across the large geographic range of $P$. cinereus, they also demonstrate the breadth of EEB fundamental phenomena which can be studied using $P$. cinereus.

5. P. cinereus falls short of model system status due to lack of infrastructure, lack of community ethos, and lack of investment by funding agencies.

6. The collaborative research network SPARCnet follows in the footsteps of other coordinated distributed experiments to cohesively and repeatedly study $P$. cinereus populations across the geographic range. Local variation is expected and embraced as we leverage our intellectually- and geographicallydistributed network to illuminate fundamental phenomena in EEB through the eyes of a woodland salamander. 


\section{Acknowledgements}

This manuscript is the product of a Summer 2020 Virtual Lab Meeting which met weekly on Zoom as a way to remain engaged in research at the start of the COVID-19 pandemic. We thank members of SPARCnet for their support, discussion, and comments on earlier versions of the manuscript, especially J.E. Fleming and E.H.C. Grant. M.C.F.-R., K.L.G., and L.S.M. were supported by a collaborative National Science Foundation Improving Undergraduate STEM Education grant (DUE Award \#s: 1914798, 1914727, 1914722, respectively). M.C.F.-R. was additionally supported by Bridgewater State University's Center for the Advancement of Research and Scholarship Faculty and Librarian Research Grant and the Bridgewater State University Office of Undergraduate Research. S.R.G. and A.K.I. were supported by the Urban Coast Institute and the Summer Research Program at Monmouth University. M.A.H. was supported by Arts \& Sciences Summer Research Fellowship from the University of Richmond. T.J.H.M. and K.N.S. were supported by Richard King Mellon Foundation Freshwater Research Institute funds for summer research at Susquehanna University. A.S. was supported by an Adrian Tinsley Program Summer Grant from the Bridgewater State University Office of Undergraduate Research. M.S. was supported by the BEACON Center for the Study of Evolution in Action at Michigan State University.

\section{Author Contributions}

M.C.F.-R. conceived of the review, conducted the literature searches on salamander families, genera, and species, performed the GIS analyses, and prepared the $P$. cinereus article list for the group effort to categorize and summarize. All authors made substantial contributions to retrieving sources on $P$. cinereus, categorizing and summarizing these sources, planning and writing the manuscript. M.C.F.-R. combined the written summaries and cross-checked all sources prior to submission.

\section{Literature Cited}

Aasen, G. \& Reichenbach, N. (2004) Is the red-backed salamander (Plethodon cinereus) encroaching upon populations of the peaks of otter salamander (P. hubrichti)? Catesbeiana 24, 17-20.

Acord, M.A., Anthony, C.D. \& Hickerson, C.-A.M. (2013) Assortative Mating in a Polymorphic Salamander. Copeia 2013, 676-683.

Adams, D.C. (2000) Divergence of trophic morphology and resource use among populations of Plethodon cinereus and P. hoffmani in Pennsylvania - A possible case of character displacement. In Biology of Plethodontid Salamanders (eds R.C. Bruce, R.G. Jaeger \& L.D. Houck), pp. 383-395. Springer Science+.

Adams, D.C. (2011) Quantitative Genetics and Evolution of Head Shape in Plethodon Salamanders. Evolutionary Biology 38, 278.

Adams, D.C. \& Rohlf, F.J. (2000) Ecological character displacement in Plethodon: Biomechanical differences found from a geometric morphometric study. Proceedings of the National Academy of Sciences 97, 41064111.

Adams, D.C., West, M.E. \& Collyer, M.L. (2007) Location-specific sympatric morphological divergence as a possible response to species interactions in West Virginia Plethodon salamander communities. Journal of Animal Ecology 76, 289-295.

AmphibiaWeb (2020) AmphibiaWeb: Information on amphibian biology and conservation. https:// amphibiaweb.org [accessed 9 June 2020].

Anderson, J.T., Solis, A.B. \& Osbourne, J.D. (2013) Herpetofaunal abundance in forested edge and interior locations of West Virginia. In: Miller, Gary W.; Schuler, Thomas M.; Gottschalk, Kurt W.; Brooks, John 
R.; Grushecky, Shawn T.; Spong, Ben D.; Rentch, James S., eds. Proceedings, 18th Central Hardwood Forest Conference; 2012 March 26-28; Morgantown, WV; Gen. Tech. Rep. NRS-P-117. Newtown Square, PA: U.S. Department of Agriculture, Forest Service, Northern Research Station: 474-486. 117, 474-486.

Angleberger, M.A.P. \& Chinnici, J.P. (1975) Dimorphism in the red-backed salamander Plethodon cinereus at Mountain Lake Virginia USA. Virginia Journal of Science 26, 153-158.

Ankeny, R.A. \& Leonelli, S. (2011) What's so special about model organisms? Studies in History and Philosophy of Science Part A 42, 313-323.

Anthony, C.D., Hickerson, C.-A.M. \& Venesky, M.D. (2007) Responses of juvenile terrestrial salamanders to introduced (Lithobius forficatus) and native centipedes (Scolopocryptops sexspinosus). Journal of Zoology 271, 54-62.

Anthony, C.D., Jaworski, K.E., Messner, M. \& Hickerson, C.-A.M. (2017) Differences in prey availability within the territories of striped and unstriped eastern red-backed salamanders (Plethodon cinereus). Herpetological Review 48, 509-514.

Anthony, C.D., Mendelson, J.R. \& Simons, R.R. (1994) Differential Parasitism by Sex on Plethodontid Salamanders and Histological Evidence for Structural Damage to the Nasolabial Groove. The American Midland Naturalist 132, 302-307.

Anthony, C.D., Venesky, M.D. \& Hickerson, C.-A.M. (2008) Ecological separation in a polymorphic terrestrial salamander. Journal of Animal Ecology 77, 646-653.

Arif, S., Adams, D. \& Wicknick, J. (2007) Bioclimatic modelling, morphology, and behaviour reveal alternative mechanisms regulating the distributions of two parapatric salamander species. Evolutionary Ecology Research 9, 843-854.

Arnold, S.J. (1977) The Evolution of Courtship Behavior in New World Salamanders with Some Comments on Old World Salamandrids. In The Reproductive Biology of Amphibians (eds D.H. Taylor \& S.I. Guttman), pp. 141-183. Springer US, Boston, MA.

Augustine, L. \& Neff, M. (2016) Determining the prevalence of Amphibian Chytrid Fungus (Batrachochytrium dendrobatidis) and Ranavirus at Long Branch Nature Center in Arlington, Virginia. Catesbeiana 36, 35-42.

Babikova, Z., Gilbert, L., Bruce, T.J.A., Birkett, M., Caulfield, J.C., Woodcock, C., Pickett, J.A. \& Johnson, D. (2013) Underground signals carried through common mycelial networks warn neighbouring plants of aphid attack. Ecology Letters 16, 835-843.

Barnes, E.M., Carter, E.L. \& Lewis, J.D. (2020) Predicting Microbiome Function Across Space Is Confounded by Strain-Level Differences and Functional Redundancy Across Taxa. Frontiers in Microbiology 11, 101.

Bayer, C.S.O., Sackman, A.M., Bezold, K., Cabe, P.R. \& Marsh, D.M. (2012) Conservation genetics of an endemic mountaintop salamander with an extremely limited range. Conservation Genetics 13, 443-454.

Bazar, M.A., Quinn, M.J., Mozzachio, K., Bleiler, J.A., Archer, C.R., Phillips, C.T. \& Johnson, M.S. (2009) Toxicological Responses of Red-Backed Salamanders (Plethodon cinereus) to Soil Exposures of Copper. Archives of Environmental Contamination and Toxicology 57, 116-122.

Bazar, M.A., Quinn, M.J., Mozzachio, K., Bleiler, J.A., Archer, C.R., Phillips, C.T. \& Johnson, M.S. (2010) Toxicological Responses of Red-Backed Salamander (Plethodon cinereus) Exposed to Aged and Amended Soils Containing Lead. Archives of Environmental Contamination and Toxicology 58, 1040-1047.

Bazar, M.A., Quinn, M.J., Mozzachio, K. \& Johnson, M.S. (2008) Toxicological responses of red-backed salamanders (Plethodon cinereus) to subchronic soil exposures of 2,4,6-trinitrotoluene. Environmental Toxicology and Chemistry 27, 1393-1398. 
Becker, M.H., Brucker, R.M., Schwantes, C.R., Harris, R.N. \& Minbiole, K.P.C. (2009) The Bacterially Produced Metabolite Violacein Is Associated with Survival of Amphibians Infected with a Lethal Fungus. Applied and Environmental Microbiology 75, 6635-6638.

Becker, S., Moorman, C., DePerno, C. \& Simons, T. (2013) Quantifiable Long-term Monitoring on Parks and Nature Preserves. Southeastern Naturalist 12, 339-352.

Begon, M., Townsend, C.R. \& Harper, J.L. (2006) Ecology: From Individuals to Ecosystems 4th edition. Wiley-Blackwell, Malden, MA.

Beier, C.M., Woods, A.M., Hotopp, K.P., Gibbs, J.P., Mitchell, M.J., Dovčiak, M., Leopold, D.J., Lawrence, G.B. \& Page, B.D. (2012) Changes in faunal and vegetation communities along a soil calcium gradient in northern hardwood forests. Canadian Journal of Forest Research 42, 1141-1152.

Bellocq, M.I., Kloosterman, K. \& Smith, S.M. (2000) The diet of coexisting species of amphibians in Canadian jack pine forests. Herpetological Journal 10, 63-68.

Bennett, R.M., Ross, R.M., Lellis, W.A. \& Redell, L.A. (2003) Terrestrial salamander preference for artificial cover objects made from four species of wood. Journal of the Pennsylvania Academy of Science 76, 77-79.

Bergeron, C.M., Bodinof, C.M., Unrine, J.M. \& Hopkins, W.A. (2010a) Mercury accumulation along a contamination gradient and nondestructive indices of bioaccumulation in amphibians. Environmental Toxicology and Chemistry 29, 980-988.

Bergeron, C.M., Bodinof, C.M., Unrine, J.M. \& Hopkins, W.A. (2010b) Bioaccumulation and maternal transfer of mercury and selenium in amphibians. Environmental Toxicology and Chemistry 29, 989-997.

Best, M.L. \& Welsh, H.H. (2014) The trophic role of a forest salamander: impacts on invertebrates, leaf litter retention, and the humification process. Ecosphere 5, 16.

Biddle, J.R., Grant, A.H. \& Liebgold, E.B. (2017) Factors Affecting the Growth of Eastern Red-backed Salamanders, Plethodon cinereus. Herpetologica 73, 89-93.

Bishop, S.J. (1941) The salamanders of New York. New York State Museum Bulletin 324, 1-365.

Blankers, T., Adams, D.C. \& Wiens, J.J. (2012) Ecological radiation with limited morphological diversification in salamanders. Journal of Evolutionary Biology 25, 634-646.

Bolek, M.G. \& Coggins, J.R. (1998) Helminth parasites of the spotted salamander Ambystoma maculatum and red-backed salamander Plethodon c. cinereus from Northwestern Wisconsin. Journal of the Helminthological Society of Washington 65, 98-102.

Bondi, C.A., Beier, C.M., Ducey, P.K., Lawrence, G.B. \& Bailey, S. (2016) Can the eastern red-backed salamander (Plethodon cinereus) persist in an acidified landscape? Ecosphere 7, e01318.

Bondi, C.A., Beier, C.M., Fierke, M.K. \& Ducey, P.K. (2019) The role of feeding strategy in the tolerance of a terrestrial salamander (Plethodon cinereus) to biogeochemical changes in northern hardwood forests. Canadian Journal of Zoology 97, 281-293.

Bondi, C.A., Green, S. \& Beier, C.M. (2015) Evaluation of Gastric Lavage Method for Diet Analysis of the Eastern Red-backed Salamander (Plethodon cinereus). Herpetological Review 46, 196-199.

Bonin, J. \& Bachand, Y. (1997) The use of artificial covers to survey terrestrial salamanders in Quebec. Herpetological Conservation 1, 175-179.

Borer, E.T., Harpole, W.S., Adler, P.B., Lind, E.M., Orrock, J.L., Seabloom, E.W. \& Smith, M.D. (2014) Finding generality in ecology: a model for globally distributed experiments. Methods in Ecology and Evolution 5, 65-73. 
Brenner, F.J., Smith, E.D. \& Marr, M.J. (2005) Factors affecting the distribution of redback salamanders (Plethodon cinereus) in a second growth forest community. Journal of the Pennsylvania Academy of Science 78, 53-56.

Brooks, R.T. (1999) Residual Effects of Thinning and High White-Tailed Deer Densities on Northern Redback Salamanders in Southern New England Oak Forests. The Journal of Wildlife Management 63, 1172-1180.

Brooks, R.T. (2001) Effects of the removal of overstory hemlock from hemlock-dominated forests on eastern redback salamanders. Forest Ecology and Management 149, 197-204.

Brown, G.S., Pollock, L., DeWitt, P.D. \& Dawson, N. (2020) Responses of terrestrial animals to forest characteristics and climate reveals ecological indicators for sustaining wildlife in managed forests. Forest Ecology and Management 459, 117854.

Brucker, R.M., Baylor, C.M., Walters, R.L., Lauer, A., Harris, R.N. \& Minbiole, K.P.C. (2008a) The identification of 2,4-diacetylphloroglucinol as an antifungal metabolite produced by cutaneous bacteria of the salamander Plethodon cinereus. Journal of Chemical Ecology 34, 39-43.

Brucker, R.M., Harris, R.N., Schwantes, C.R., Gallaher, T.N., Flaherty, D.C., Lam, B.A. \& Minbiole, K.P.C. (2008b) Amphibian chemical defense: antifungal metabolites of the microsymbiont Janthinobacterium lividum on the salamander Plethodon cinereus. Journal of Chemical Ecology 34, 1422-1429.

Brunges, H.J., Dunn, J.P., Helder, D.R. \& Otieno, S. (2020) Effects of invasive earthworm feeding guilds and their interactions with physiographic conditions on the relative abundance and distribution of woodland salamanders. Herpetological Conservation and Biology 15, 16-24.

Burger, J.W. (1935) Plethodon cinereus (Green) in Eastern Pennsylvania and New Jersey. The American Naturalist 69, 578-586.

Burgett, A.A. \& Smith, G.R. (2012) Differential Responses of Eastern Red-backed Salamanders (Plethodon cinereus) to Conspecifics and Centipedes. Current Herpetology 31, 78-86.

Burton, T.M. \& Likens, G.E. (1975a) Salamander Populations and Biomass in the Hubbard Brook Experimental Forest, New Hampshire. Copeia 1975, 541-546.

Burton, T.M. \& Likens, G.E. (1975b) Energy Flow and Nutrient Cycling in Salamander Populations in the Hubbard Brook Experimental Forest, New Hampshire. Ecology 56, 1068-1080.

Cabe, P.R., Page, R.B., Hanlon, T.J., Aldrich, M.E., Connors, L. \& Marsh, D.M. (2007) Fine-scale population differentiation and gene flow in a terrestrial salamander (Plethodon cinereus) living in continuous habitat. Heredity 98, 53-60.

Cáceres-Charneco, R.I. \& Ransom, T.S. (2010) The influence of habitat provisioning: use of earthworm burrows by the terrestrial salamander, Plethodon cinereus. Population Ecology 52, 517-526.

Cameron, A.C., Anderson, J.J. \& Page, R.B. (2017) Assessment of intra and interregional genetic variation in the Eastern Red-backed Salamander, Plethodon cinereus, via analysis of novel microsatellite markers. PLOS ONE 12, e0186866.

Cameron, A.C., Hickerson, C.-A.M. \& Anthony, C.D. (2016) Plethodon cinereus (Eastern Red-backed Salamander) Not Affected by Long-term Exposure to Soil Liming. Northeastern Naturalist 23, 88-99.

Carfioli, M.A., Tiebout, H.M., Pagano, S.A., Heister, K.M. \& Lutcher, F.C. (2000) Monitoring Plethodon cinereus populations - Field tests of experimental coverboard designs. In Biology of Plethodontid Salamanders (eds R.C. Bruce, R.G. Jaeger \& L.D. Houck), pp. 463-475. Springer Science+.

Carlson, B.E., Thawley, C.J. \& Graham, S.P. (2016) Natural history of the valley and ridge salamander (Plethodon hoffmani): demography, movement, microhabitats, and abundance. Herpetological Conservation and Biology 11, 315-327. 
Carlson, T.A. (Yoder) \& Szuch, E.J. (2007) Un-weathered (new) artificial cover objects effectively sample plethodontid salamanders in Michigan. Herpetological Review 38, 412-415.

Caro, T. (2010) Conservation by Proxy: Indicator, Umbrella, Keystone, Flagship, and Other Surrogate Species. Island Press.

Carpenter, D.W., Jung, R.E. \& Sites, J.W. (2001) Conservation genetics of the endangered Shenandoah salamander (Plethodon shenandoah, Plethodontidae). Animal Conservation Forum 4, 111-119.

Caruso, N.M., Sears, M.W., Adams, D.C. \& Lips, K.R. (2014) Widespread rapid reductions in body size of adult salamanders in response to climate change. Global Change Biology 20, 1751-1759.

Chalmers, R.J. \& Loftin, C.S. (2010) Plethodon cinereus (Eastern Red-backed Salamander). Wetland Habitat. Herpetological Review 41, 468-469.

Chambert, T., Grant, E.H.C., Miller, D.A.W., Nichols, J.D., Mulder, K.P. \& Brand, A.B. (2018) Twospecies occupancy modelling accounting for species misidentification and non-detection. Methods in Ecology and Evolution 9, 1468-1477.

Chouinard, A.J. (2012) Rapid onset of mate quality assessment via chemical signals in a woodland salamander (Plethodon cinereus). Behavioral Ecology and Sociobiology 66, 765-775.

Church, D.R. \& Okazaki, R.K. (2002) Seasonal variation of plasma testosterone titres in male redback salamanders, Plethodon cinereus. Amphibia-Reptilia 23, 93-97.

Ciul, K., Simpson, L., Smith, G.R. \& Rettig, J.E. (2010) Evaluation of the rate of artificial coverboard use by the salamander, Plethodon cinereus, in the vicinity of natural cover objects. Bulletin of the Maryland Herpetological Society 46, 17-19.

Clarivate (2021) KeyWords Plus generation, creation, and changes. https://support.clarivate. $\mathrm{com} /$ ScientificandAcademicResearch/s/article/KeyWords-Plus-generation-creation-andchanges? language $=e n_{-}$US [accessed 12 July 2021].

Clutton-Brock, T. \& Sheldon, B.C. (2010) Individuals and populations: the role of long-term, individualbased studies of animals in ecology and evolutionary biology. Trends in Ecology 83 Evolution 25, 562-573.

Connette, G.M., Crawford, J.A. \& Peterman, W.E. (2015) Climate change and shrinking salamanders: alternative mechanisms for changes in plethodontid salamander body size. Global Change Biology 21, 2834-2843.

Connors, L.M. \& Cabe, P.R. (2003) Isolation of dinucleotide microsatellite loci from red-backed salamander (Plethodon cinereus). Molecular Ecology Notes 3, 131-133.

Cosentino, B.J. \& Brubaker, K.M. (2018) Effects of land use legacies and habitat fragmentation on salamander abundance. Landscape Ecology 33, 1573-1584.

Cosentino, B.J. \& Droney, D.C. (2016) Movement behaviour of woodland salamanders is repeatable and varies with forest age in a fragmented landscape. Animal Behaviour 121, 137-146.

Cosentino, B.J., Moore, J.-D., Karraker, N.E., Ouellet, M. \& Gibbs, J.P. (2017) Evolutionary response to global change: Climate and land use interact to shape color polymorphism in a woodland salamander. Ecology and Evolution 7, 5426-5434.

Cote, G., Getty, S., Vokoun, A., Carmichael, S., Hunt, H., Letton, N., McEntire, K.D., Wooten, J.A. \& Camp, C.D. (2016) A test of scent-trailing as a contributing factor in the climbing behaviour of the redback salamander (Plethodon cinereus). Amphibia-Reptilia 37, 111-116.

Cove, M.V., Kays, R., Bontrager, H., Bresnan, C., Lasky, M., Frerichs, T., Klann, R., Lee, T.E., Crockett, S.C., Crupi, A.P., Weiss, K.C.B., Rowe, H., Sprague, T., Schipper, J., Tellez, C., et al. (2021) Snapshot USA 2019: a coordinated national camera trap survey of the United States. Ecology 102, e03353. 
CRC (2014) CRC 806 Database: Maps. https://crc806db.uni-koeln.de/layer/show/6 [accessed 12 July 2021].

Crespi, E.J. \& Lessig, H. (2004) Mothers influence offspring body size through post-oviposition maternal effects in the redbacked salamander, Plethodon cinereus. Oecologia 138, 306-311.

Culp, C.E., Falkinham, J.O., III \& Belden, L.K. (2007) Identification of the natural bacterial microflora on the skin of eastern newts, bullfrog tadpoles and redback salamanders. Herpetologica 63, 66-71.

Cusi, J.C., Gagliardi-Urrutia, G., Brcko, I.C., Wake, D.B. \& May, R.V. (2020) Taxonomic status of the Neotropical salamanders Bolitoglossa altamazonica and Bolitoglossa peruviana (Amphibia: Caudata: Plethodontidae), with the description of a new species from Northern Peru. Zootaxa 4834, 3.

Dallalio, E.A., Brand, A.B. \& Grant, E.H.C. (2017) Climate-Mediated Competition in a High-Elevation Salamander Community. Journal of Herpetology 51, 190-196.

Davic, R.D. \& Welsh, H.H. (2004) On the Ecological Roles of Salamanders. Annual Review of Ecology, Evolution, and Systematics 35, 405-434.

Davis, A., Milanovich, J., DeVore, J. \& Maerz, J. (2009a) An investigation of factors influencing erythrocyte morphology of red-backed salamanders (Plethodon cinereus). Animal Biology 59, 201-209.

Davis, A.K. \& Cecala, K. (2010) Intraerythrocytic rickettsial inclusions in Ocoee salamanders (Desmognathus ocoee): prevalence, morphology, and comparisons with inclusions of Plethodon cinereus. Parasitology Research 107, 363-367.

Davis, A.K., DeVore, J.L., Milanovich, J.R., Cecala, K., Maerz, J.C. \& Yabsley, M.J. (2009b) New Findings from an Old Pathogen: Intraerythrocytic Bacteria (Family Anaplasmatacea) in Red-Backed Salamanders Plethodon cinereus. EcoHealth 6, 219-228.

Davis, A.K. \& Milanovich, J.R. (2010) Lead-phase and red-stripe color morphs of red-backed salamanders Plethodon cinereus differ in hematological stress indices: A consequence of differential predation pressure? Current Zoology 56, 238-243.

Dawley, E.M., Fingerlin, A., Hwang, D., John, S.S. \& Stankiewicz, C.A. (2000) Seasonal Cell Proliferation in the Chemosensory Epithelium and Brain of Red-Backed Salamanders, Plethodon cinereus. Brain, Behavior and Evolution 56, 1-13.

Dawley, E.M., Nelsen, M., Lopata, A., Schwartz, J. \& Bierly, A. (2006) Cell Birth and Survival following Seasonal Periods of Cell Proliferation in the Chemosensory Epithelia of Red-Backed Salamanders, Plethodon cinereus. Brain, Behavior and Evolution 68, 26-36.

Deitloff, J., Adams, D.C., Olechnowski, B.F.M. \& Jaeger, R.G. (2008) Interspecific Aggression in Ohio Plethodon: Implications for Competition. Herpetologica 64, 180-188.

Deitloff, J., Church, J.O., Adams, D.C. \& Jaeger, R.G. (2009) Interspecific Agonistic Behaviors in a Salamander Community: Implications for Alpha Selection. Herpetologica 65, 174-182.

Deitloff, J., Petersen, J.D. \& Adams, D.C. (2013) Complex Species Interactions Lead to Unpredictable Outcomes in Plethodon. Herpetologica 69, 1-10.

deMaynadier, P.G. \& Hunter, M.L. (1995) The relationship between forest management and amphibian ecology: a review of the North American literature. Environmental Reviews 3, 230-261.

deMaynadier, P.G. \& Hunter, M.L. (1998) Effects of Silvicultural Edges on the Distribution and Abundance of Amphibians in Maine. Conservation Biology 12, 340-352.

Devreotes, P. (1989) Dictyostelium discoideum: a model system for cell-cell interactions in development. Science 245, 1054-1058. 
Dewsbury, D.A. (2012) A history of the behavior program at the Jackson Laboratory: An overview. Journal of Comparative Psychology 126, 31-44.

Dizney, L., Connors, P.K., Varner, J., Duggan, J.M., Lanier, H.C., Erb, L.P., Flaherty, E.A., Yahnke, C.J. \& Hanson, J.D. (2020) An Introduction to the Squirrel-Net Teaching Modules. CourseSource. https://doi. org/10.24918/cs.2020.26 [accessed 28 July 2021].

Drysdale, R.A., Crosby, M.A., \& The FlyBase Consortium (2005) FlyBase: genes and gene models. Nucleic Acids Research 33, D390-D395.

Ducey, P.K., Messere, M., Lapoint, K. \& Noce, S. (1999) Lumbricid Prey and Potential Herpetofaunal Predators of the Invading Terrestrial Flatworm Bipalium adventitium (Turbellaria: Tricladida: Terricola). The American Midland Naturalist 141, 305-314.

Duellman, W.E. \& Trueb, L. (1986) Biology of Amphibians First edition. McGraw Hill, New York.

Duffy, M.A., García-Robledo, C., Gordon, S.P., Grant, N.A., Green, D.A., Kamath, A., Penczykowski, R.M., Rebolleda-Gómez, M., Wale, N. \& Zaman, L. (2021) Model Systems in Ecology, Evolution, and Behavior: A Call for Diversity in Our Model Systems and Discipline. The American Naturalist 198, 53-68.

Duguay, J.P. \& Wood, P.B. (2002) Salamander Abundance in Regenerating Forest Stands on the Monongahela National Forest, West Virginia. Forest Science 48, 331-335.

Duhaime-Ross, A., Martel, G. \& Laberge, F. (2013) Sensory determinants of agonistic interactions in the red-backed salamander, Plethodon cinereus. Behaviour 150, 1467-1489.

Dyal, L.A. (2006) Novel Courtship Behaviors in Three Small Eastern Plethodon Species. Journal of Herpetology 40, 55-65.

Ellison, A., Zamudio, K., Lips, K. \& Muletz-Wolz, C. (2020) Temperature-mediated shifts in salamander transcriptomic responses to the amphibian-killing fungus. Molecular Ecology 29, 325-343.

ERENweb.org (2021) Mission and Goals. EREN - Ecological Research as Education Network. https:// erenweb.org/about/mission-and-goals/ [accessed 28 July 2021].

Evans, A.E., Forester, B.R., Jockusch, E.L. \& Urban, M.C. (2018) Salamander morph frequencies do not evolve as predicted in response to 40 years of climate change. Ecography 41, 1687-1697.

Evans, A.E., Urban, M.C. \& Jockusch, E.L. (2020) Developmental temperature influences color polymorphism but not hatchling size in a woodland salamander. Oecologia 192 909-918.

Faragher, S.G. \& Jaeger, R.G. (1997) Distributions of Adult and Juvenile Red-Backed Salamanders: Testing New Hypotheses regarding Territoriality. Copeia 1997, 410-414.

Farallo, V.R. \& Miles, D.B. (2016) The Importance of Microhabitat: A Comparison of Two Microendemic Species of Plethodon to the Widespread P. cinereus. Copeia 104, 67-77.

Fisher-Reid, M.C., Engstrom, T.N., Kuczynski, C.A., Stephens, P.R. \& Wiens, J.J. (2013) Parapatric divergence of sympatric morphs in a salamander: incipient speciation on Long Island? Molecular Ecology 22, 4681-4694.

Fisher-Reid, M.C., Kozak, K.H. \& Wiens, J.J. (2012) How Is the Rate of Climatic-Niche Evolution Related to Climatic-Niche Breadth? Evolution 66, 3836-3851.

Fisher-Reid, M.C. \& Wiens, J.J. (2011) What are the consequences of combining nuclear and mitochondrial data for phylogenetic analysis? Lessons from Plethodon salamanders and 13 other vertebrate clades. BMC Evolutionary Biology 11, 300.

Fisher-Reid, M.C. \& Wiens, J.J. (2015) Is geographic variation within species related to macroevolutionary patterns between species? Journal of Evolutionary Biology 28, 1502-1515. 
Fitzpatrick, B.M., Shook, K. \& Izally, R. (2009) Frequency-dependent selection by wild birds promotes polymorphism in model salamanders. BMC Ecology 9, 12.

Fleming, J., Grant, E.H.C., Sterrett, S.C. \& Sutherland, C. (2021) Experimental evaluation of spatial capture-recapture study design. Ecological Applications, doi:10.1002/eap.2419.

Fleming, J., Sutherland, C., Sterrett, S.C. \& Grant, E.H.C. (2020) A latent process model approach to improve the utility of indicator species. Oikos 129, 1753-1762.

Fleming, M.M., Mills, L.B., Russell, J.K., Smith, G.R. \& Rettig, J.E. (2011) Effects of Trails on Eastern Redback Salamander (Plethodon cinereus Green). Herpetology Notes 4, 229-232.

Fontaine, S.S., Novarro, A.J. \& Kohl, K.D. (2018) Environmental temperature alters the digestive performance and gut microbiota of a terrestrial amphibian. Journal of Experimental Biology 221, jeb187559.

Forester, D.C. \& Anders, C. (2000) Contributions to the life history of the redback salamander, Plethodon cinereus - Preliminary evidence for kin recognition. In Biology of Plethodontid Salamanders (eds R.C. Bruce, R.G. Jaeger \& L.D. Houck), pp. 407-416. Springer Science+.

Fraser, D.F. (1980) On the environmental control of oocyte maturation in a plethodontid salamander. Oecologia 46, 302-307.

Fraser, L.H., Henry, H.A., Carlyle, C.N., White, S.R., Beierkuhnlein, C., Cahill, J.F., Casper, B.B., Cleland, E., Collins, S.L., Dukes, J.S., Knapp, A.K., Lind, E., Long, R., Luo, Y., Reich, P.B., et al. (2013) Coordinated distributed experiments: an emerging tool for testing global hypotheses in ecology and environmental science. Frontiers in Ecology and the Environment 11, 147-155.

Fredericks, L.P. \& Dankert, J.R. (2000) Antibacterial and hemolytic activity of the skin of the terrestrial salamander, Plethodon cinereus. Journal of Experimental Zoology 287, 340-345.

Frisbie, M.P. \& Wyman, R.L. (1991) The Effects of Soil pH on Sodium Balance in the Red-Backed Salamander, Plethodon cinereus, and Three Other Terrestrial Salamanders. Physiological Zoology 64, 1050-1068. The University of Chicago Press.

Gade, M.R., Donlon, K.C., Gould, P.R. \& Wittum, R.R. (2017) Female Plethodon cinereus cannibalism of an adult conspecific. Herpetology Notes 10, 615-615.

Gaglione, C.M., O’Meara, E.M., Pence, K.L., Petterson, A.C., Smith, G.R. \& Rettig, J.E. (2011) Red-backed Salamander, Plethodon cinereus (Green, 1818): avoidance of urea. Herpetology Notes 4, 275-277.

Gall, S.B., Anthony, C.D. \& Wicknick, J.A. (2003) Do Behavioral Interactions between Salamanders and Beetles Indicate a Guild Relationship? The American Midland Naturalist 149, 363-374.

Gartner, T.B., Thomas, C.L., Geedey, K., Bjorgo-Thorne, K., Simmons, J.A., Shea, K.L., Dosch, J.J. \& Zimmermann, C.R. (2020) Strategies for Incorporating Long-Term, Distributed-Network Research Projects into the Undergraduate Curriculum: Lessons from the Ecological Research as Education Network's Decomposition Project. The American Biology Teacher 82, 142-148.

Gertzog, B.J., Kaplan, L.J., Nichols, D., Smith, G.R. \& Rettig, J.E. (2011) Avoidance of three herbicide formulations by eastern red-backed salamanders (Plethodon cinereus). Herpetological Conservation and Biology 6, 237-241.

Gibbons, M.E., Ferguson, A.M. \& Lee, D.R. (2005) Both learning and heritability affect foraging behaviour of red-backed salamanders, Plethodon cinereus. Animal Behaviour 69, 721-732.

Gibbs, J.P. (1998a) Amphibian Movements in Response to Forest Edges, Roads, and Streambeds in Southern New England. The Journal of Wildlife Management 62, 584-589.

Gibbs, J.P. (1998b) Distribution of woodland amphibians along a forest fragmentation gradient. Landscape Ecology 13, 263-268. 
Gibbs, J.P. (1998c) Genetic structure of redback salamander Plethodon cinereus populations in continuous and fragmented forests. Biological Conservation 86, 77-81.

Gibbs, J.P. \& Karraker, N.E. (2006) Effects of Warming Conditions in Eastern North American Forests on Red-Backed Salamander Morphology. Conservation Biology 20, 913-917.

Gilbert, K.J., Fahey, T.J., Maerz, J.C., Sherman, R.E., Bohlen, P., Dombroskie, J.J., Groffman, P.M. \& Yavitt, J.B. (2014) Exploring carbon flow through the root channel in a temperate forest soil food web. Soil Biology and Biochemistry 76, 45-52.

Gillette, J.R., Kolb, S.E., Smith, J.A. \& Jaeger, R.G. (2000) Pheromonal attractions to particular males by female redback salamanders (Plethodon cinereus). In Biology of Plethodontid Salamanders (eds R.C. Bruce, R.G. Jaeger \& L.D. Houck), pp. 431-441. Springer Science+ .

Gillette, J.R. \& Peterson, M.G. (2001) The benefits of transparency: Candling as a simple method for determining sex in red-backed salamanders (Plethodon cinereus). Herpetological Review 32, 233.

Gillilland, M.G. \& Muzzall, P.M. (2002) Amphibians, Trematodes, and Deformities: An Overview from Southern Michigan. Comparative Parasitology 69, 81-85.

Gordon, A., Wintle, B.A., Bekessy, S.A., Pearce, J.L., Venier, L.A. \& Wilson, J.N. (2012) The use of dynamic landscape metapopulation models for forest management: a case study of the red-backed salamander. Canadian Journal of Forest Research 42, 1091-1106.

Gorgolewski, A., Caspersen, J., Hazlett, P., Jones, T., Tran, H. \& Basiliko, N. (2016) Responses of Eastern Red-backed Salamander (Plethodon cinereus) abundance 1 year after application of wood ash in a northern hardwood forest. Canadian Journal of Forest Research 46, 402-409.

Graeter, G.J., Rothermel, B.B. \& Gibbons, J.W. (2008) Habitat Selection and Movement of Pond-Breeding Amphibians in Experimentally Fragmented Pine Forests. The Journal of Wildlife Management 72, 473-482.

Grant, A.H. \& Liebgold, E.B. (2017) Color-Biased Dispersal Inferred by Fine-Scale Genetic Spatial Autocorrelation in a Color Polymorphic Salamander. Journal of Heredity 108, 588-593.

Grant, A.H., Ransom, T.S. \& Liebgold, E.B. (2018) Differential Survival and the Effects of Predation on a Color Polymorphic Species, the Red-Backed Salamander (Plethodon cinereus). Journal of Herpetology 52, $127-135$.

Grant, E.H.C. (2015) Please don't misuse the museum: 'declines' may be statistical. Global Change Biology 21, 1018-1024.

Gratwicke, B., Evans, M., Grant, E.H.C., Greathouse, J., McShea, W.J., Rotzel, N. \& Fleischer, R.C. (2011) Low Prevalence of Batrachochytrium dendrobatidis Detected in Appalachian Salamanders from Warren County, Virginia, USA. Herpetological Review 42, 217-219.

Graves, B.M. \& Quinn, V.S. (2000) Temporal Persistence of Alarm Pheromones in Skin Secretions of the Salamander, Plethodon cinereus. Journal of Herpetology 34, 287-291.

Greenhalgh, T. \& Peacock, R. (2005) Effectiveness and efficiency of search methods in systematic reviews of complex evidence: audit of primary sources. BMJ 331, 1064-1065.

Greenwald, K.R., Petit, L.J. \& Waite, T.A. (2008) Indirect Effects of a Keystone Herbivore Elevate Local Animal Diversity. The Journal of Wildlife Management 72, 1318-1321.

Grizzell, R.A. (1949) The hibernation site of three snakes and a salamander. Copeia 1949, 231-232.

Grover, M.C. (1998) Influence of Cover and Moisture on Abundances of the Terrestrial Salamanders Plethodon cinereus and Plethodon glutinosus. Journal of Herpetology 32, 489-497. 
Grover, M.C. (2000) Determinants of Salamander Distributions along Moisture Gradients. Copeia 2000, $156-168$.

Grover, M.C. (2006) Comparative effectiveness of nighttime visual encounter surveys and cover object searches in detecting salamanders. Herpetological Conservation and Biology 1, 93-99.

Grover, M.C. \& Wilbur, H.M. (2002) Ecology of Ecotones: Interactions Between Salamanders on a Complex Environmental Gradient. Ecology 83, 2112-2123.

Guffey, C., MaKinster, J.G. \& Jaeger, R.G. (1998) Familiarity Affects Interactions between Potentially Courting Territorial Salamanders. Copeia 1998, 205-208.

Hantak, M.M. \& Kuchta, S.R. (2018) Predator perception across space and time: relative camouflage in a colour polymorphic salamander. Biological Journal of the Linnean Society 123, 21-33.

Hantak, M.M., Kuchta, S.R., Hickerson, C.-A.M. \& Anthony, C.D. (2015) Plethodon cinereus (Eastern Redbacked Salamander). Morphology. Herpetological Review 46, 409-410.

Hantak, M.M., Page, R.B., Converse, P.E., Anthony, C.D., Hickerson, C.-A.M. \& Kuchta, S.R. (2019) Do genetic structure and landscape heterogeneity impact color morph frequency in a polymorphic salamander? Ecography 42, 1383-1394.

Hantak, M.M., Paluh, D.J. \& Hickerson, C.-A.M. (2016) Comparison of the Diets of Sympatric Erythristic and Striped Morphs of Plethodon cinereus (Eastern Red-backed Salamander).Northeastern Naturalist 23, 219-228.

Harpole, D.N. \& Haas, C.A. (1999) Effects of seven silvicultural treatments on terrestrial salamanders. Forest Ecology and Management 114, 349-356.

Harris, R.N., James, T.Y., Lauer, A., Simon, M.A. \& Patel, A. (2006) Amphibian Pathogen Batrachochytrium dendrobatidis Is Inhibited by the Cutaneous Bacteria of Amphibian Species. EcoHealth 3, 53.

Hartzell, S.M. (2019) A Herpetological Survey of the Chincoteague Bay Field Station Campus in Accomack County, Virginia. Catesbeiana 39, 10-14.

Heatwole, H. (1960) Burrowing Ability and Behavioral Responses to Desiccation of the Salamander, Plethodon cinereus. Ecology 41, 661-668.

Hecker, L., Madison, D.M., Dapson, R.W. \& Holzherr, V. (2003) Presence of Modified Serous Glands in the Caudal Integument of the Red-Backed Salamander (Plethodon cinereus). Journal of Herpetology 37, 732-736.

Hedeen, S.E. (2000) Influence of topography on local distributions of Plethodon cinereus and P. richmondi (Plethodontidae) in Northern Kentucky and Southwestern Ohio. Journal of the Kentucky Academy of Science 61, 6-9.

Hedrick, B.P., Bancroft, G., Riand, F. \& Sutherland, C. (2021) Effects of Coverboard Age and Aging on Salamander Usage. Herpetological Review 52, 6-12.

Heemeyer, J.L., Homyack, J.A. \& Haas, C.A. (2007) Retention and readability of visible implant elastomer marks in Eastern Red-backed Salamanders (Plethodon cinereus).Herpetological Review 38, 425-425.

Hernández-Pacheco, R., Sutherland, C., Thompson, L.M. \& Grayson, K.L. (2019) Unexpected spatial population ecology of a widespread terrestrial salamander near its southern range edge. Royal Society Open Science 6, 182192.

Hesed, K.M. (2012) Uncovering Salamander Ecology: A Review of Coverboard Design. Journal of Herpetology 46, 442-450.

Hess, A., McAllister, C., DeMarchi, J., Zidek, M., Murone, J. \& Venesky, M.D. (2015) Salamanders increase their feeding activity when infected with the pathogenic chytrid fungus Batrachochytrium dendrobatidis. 
Diseases of Aquatic Organisms 116, 205-212.

Hessel, A.L., Ryerson, W.G. \& Whitenack, L.B. (2017) Doing Without: Jump Performance After Tail Autotomy in Three Species of Plethodontid Salamanders. Herpetologica 73, 100-104.

Hickerson, C.-A.M., Anthony, C.D. \& Figura, A.M. (2018) Behavioral interactions between terrestrial salamanders and spiders: competition or intraguild predation? Ethology Ecology \& Evolution 30, 285-296.

Hickerson, C.-A.M., Anthony, C.D. \& Walton, B.M. (2012) Interactions among Forest-floor Guild Members in Structurally Simple Microhabitats. The American Midland Naturalist 168, 30-42.

Hickerson, C.-A.M., Anthony, C.D. \& Walton, B.M. (2017) Eastern Red-backed Salamanders Regulate TopDown Effects in a Temperate Forest-Floor Community. Herpetologica 73, 180-189.

Highton, R. (1977) Comparison of microgeographic variation in morphological and electrophoretic traits. Evolutionary Biology 10, 397-436.

Highton, R. (1999) Geographic Protein Variation and Speciation in the Salamanders of the Plethodon cinereus Group with the Description of Two New Species. Herpetologica 55, 43-90.

Highton, R., Hastings, A.P., Palmer, C., Watts, R., Hass, C.A., Culver, M. \& Arnold, S.J. (2012) Concurrent speciation in the eastern woodland salamanders (Genus Plethodon): DNA sequences of the complete albumin nuclear and partial mitochondrial 12s genes. Molecular Phylogenetics and Evolution 63, 278-290.

Highton, R. \& Larson, A. (1979) The Genetic Relationships of the Salamanders of the Genus Plethodon. Systematic Biology 28, 579-599.

Highton, R. \& Webster, T.P. (1976) Geographic Protein Variation and Divergence in Populations of the Salamander Plethodon cinereus. Evolution 30, 33-45.

Hobbie, J.E., Carpenter, S.R., Grimm, N.B., Gosz, J.R. \& Seastedt, T.R. (2003) The US Long Term Ecological Research Program. BioScience 53, 21-32.

Hocking, D.J. \& Babbitt, K.J. (2014) Effects of Red-Backed Salamanders on Ecosystem Functions. PLOS ONE 9, e86854.

Hocking, D.J., Babbitt, K.J. \& Yamasaki, M. (2013) Comparison of silvicultural and natural disturbance effects on terrestrial salamanders in northern hardwood forests. Biological Conservation 167, 194-202.

Homyack, J.A. \& Haas, C.A. (2009) Long-term effects of experimental forest harvesting on abundance and reproductive demography of terrestrial salamanders. Biological Conservation 142, 110-121.

Homyack, J.A., Haas, C.A. \& Hopkins, W.A. (2010) Influence of temperature and body mass on standard metabolic rate of eastern red-backed salamanders (Plethodon cinereus). Journal of Thermal Biology 35, $143-146$.

Homyack, J.A., Haas, C.A. \& Hopkins, W.A. (2011) Energetics of surface-active terrestrial salamanders in experimentally harvested forest. The Journal of Wildlife Management 75, 1267-1278.

Hughes, B.B., Beas-Luna, R., Barner, A.K., Brewitt, K., Brumbaugh, D.R., Cerny-Chipman, E.B., Close, S.L., Coblentz, K.E., de Nesnera, K.L., Drobnitch, S.T., Figurski, J.D., Focht, B., Friedman, M., Freiwald, J., Heady, K.K., et al. (2017) Long-Term Studies Contribute Disproportionately to Ecology and Policy. BioScience 67, 271-281.

Hughes, M., Petersen, R. \& Duffield, R.M. (1999) Plethodon cinereus (red-backed salamander). Habitat. Herpetological Review 30, 160-160.

Hurst, L.M. \& Smith, G.R. (2006) Response of Plethodon cinereus to chemical cues from different numbers of conspecifics. Bulletin of the Maryland Herpetological Society 42, 144-148. 
IUCN (2020) The IUCN Red List of Threatened Species. IUCN Red List of Threatened Species. Version 2020-2. https://www. iucnredlist.org/en [accessed 11 October 2020].

Ivanov, K., Lockhart, O.M., Keiper, J. \& Walton, B.M. (2011) Status of the exotic ant Nylanderia flavipes (Hymenoptera: Formicidae) in northeastern Ohio. Biological Invasions 13, 1945-1950.

Iverson, J.P. \& Smith, G.R. (2010) Substrate and cover object choice by the Red-Backed Salamander (Plethodon cinereus). Bulletin of the Maryland Herpetological Society 46, 13-16.

Jaeger, R., Gabor, C. \& Wilbur, H. (1998) An Assemblage of Salamanders in the Southern Appalachian Mountains: Competitive and Predatory Behavior. Behaviour 135, 795-821.

Jaeger, R.G. (1970) Potential Extinction Through Competition Between Two Species of Terrestrial Salamanders. Evolution 24, 632-642.

Jaeger, R.G. (1972) Food as a Limited Resource in Competition between Two Species of Terrestrial Salamanders. Ecology 53, 535-546.

Jaeger, R.G. (1980) Microhabitats of a Terrestrial Forest Salamander. Copeia 1980, 265-268.

Jaeger, R.G., Gillette, J.R. \& Cooper, R.C. (2002) Sexual coercion in a territorial salamander: males punish socially polyandrous female partners. Animal Behaviour 63, 871-877.

Jaeger, R.G., Gollmann, B., Anthony, C.D., Gabor, C.R. \& Kohn, N.R. (2016) Behavioral Ecology of the Eastern Red-backed Salamander: 50 Years of Research 1st edition. Oxford University Press, New York, NY.

Jaeger, R.G., Peterson, M.G., Gollmann, G., Gollmann, B. \& Townsend, V.R. (2001) Salamander Social Strategies: Living Together in Female-Male Pairs. Journal of Herpetology 35, 335-338.

Jaramillo, A.F., De La Riva, I., Guayasamin, J.M., Chaparro, J.C., Gagliardi-Urrutia, G., Gutiérrez, R.C., Brcko, I., Vilà, C. \& Castroviejo-Fisher, S. (2020) Vastly underestimated species richness of Amazonian salamanders (Plethodontidae: Bolitoglossa) and implications about plethodontid diversification. Molecular Phylogenetics and Evolution 149, 106841.

Jaworski, K.E., Lattanzio, M.S., Hickerson, C.-A.M. \& Anthony, C.D. (2018) Male mate preference as an agent of fecundity selection in a polymorphic salamander. Ecology and Evolution 8, 8750-8760.

Johnson, M.S., McFarland, C.A., Bazar, M.A., Quinn, M.J., LaFiandra, E.M. \& Talent, L.G. (2010) Toxicity of Octahydro-1,3,5,7-Tetranitro-1,3,5,7-Tetrazocine (HMX) in Three Vertebrate Species. Archives of Environmental Contamination and Toxicology 58, 836-843.

Johnson, M.S., Paulus, H.I., Salice, C.J., Checkai, R.T. \& Simini, M. (2004) Toxicologic and Histopathologic Response of the Terrestrial Salamander Plethodon cinereus to Soil Exposures of 1,3,5-Trinitrohexahydro1,3,5-Triazine. Archives of Environmental Contamination and Toxicology 47, 496-501.

Johnson, M.S., Suski, J. \& Bazar, M.A. (2007) Toxicological responses of red-backed salamanders (Plethodon cinereus) to subchronic soil exposures of 2,4-dinitrotoluene. Environmental Pollution (Barking, Essex: 1987) 147, 604-608.

Jongsma, G.F.M. (2012a) Plethodon cinereus (Eastern Red-backed Salamander). Morphology. Herpetological Review 43, 318-318.

Jongsma, G.F.M. (2012b) Plethodon cinereus (Eastern Red-backed Salamander). Morphology. Herpetological Review 43, 460-460.

Jongsma, G.F.M., Empey, M.A., Smme, C.M., Bennett, A.M. \& McAlpine, D.E. (2019) High prevalence of the amphibian pathogen Batrachochytrium dendrobatidis in plethodontid salamanders in protected areas in New Brunswick, Canada. Herpetological Conservation and Biology 14, 91-96. 
Jordan, M.A., Morris, D.A. \& Gibson, S.E. (2008) The influence of historical landscape change on genetic variation and population structure of a terrestrial salamander (Plethodon cinereus). (Plethodon cinereus). Conservation Genetics 10, 1647.

Jung, R.E., Ward, W.L., King, C.O. \& Weir, L.A. (2000) Plethodon cinereus (redback salamander). Predation. Herpetological Review 31, 98-99.

Karuzas, J.M., Maerz, J.C. \& Madison, D.M. (2004) An alternative hypothesis for the primary function of a proposed mate assessment behaviour in red-backed salamanders. Animal Behaviour 68, 489-494.

Keller, M., Schimel, D.S., Hargrove, W.W. \& Hoffman, F.M. (2008) A continental strategy for the national ecological observatory network. The Ecological Society of America: 282-284 .

Kerby, J.L., Richards-Hrdlicka, K.L., Storfer, A. \& Skelly, D.K. (2010) An examination of amphibian sensitivity to environmental contaminants: are amphibians poor canaries? Ecology Letters 13, 60-67.

Kerney, R. (2011) Embryonic Staging Table for a Direct-Developing Salamander, Plethodon cinereus (Plethodontidae). The Anatomical Record 294, 1796-1808.

Kerney, R.R., Blackburn, D.C., Müller, H. \& Hanken, J. (2012) Do Larval Traits Re-Evolve? Evidence from the Embryogenesis of a Direct-Developing Salamander, Plethodon cinereus. Evolution 66, 252-262.

Keyser, P.D., Sausville, D.J., Ford, W.M., Schwab, D.J. \& Brose, P.H. (2004) Prescribed fire impacts to amphibians and reptiles in shelterwood-harvested oak-dominated forests. Virginia Journal of Science 55, $159-168$.

Kight, S.L., Eadie, C., Lynch, D., Coelho, J. \& Dewera, A. (2005) Classical conditioning of red-backed salamanders, Plethodon cinereus. Bulletin of the Maryland Herpetological Society 41, 68-84.

King, R.B., Oldham, M.J., Weller, W.F. \& Wynn, D. (1997) Historic and Current Amphibian and Reptile Distributions in the Island Region of Western Lake Erie. The American Midland Naturalist 138, 153-173.

Kleeberger, S.R. \& Werner, J.K. (1982) Home Range and Homing Behavior of Plethodon cinereus in Northern Michigan. Copeia 1982, 409-415.

Klein, H.G. (1960) Population Estimate of the Red-Backed Salamander. Herpetologica 16, 52-54.

Knapp, S.M., Haas, C.A., Harpole, D.N. \& Kirkpatrick, R.L. (2003) Initial Effects of Clearcutting and Alternative Silvicultural Practices on Terrestrial Salamander Abundance. Conservation Biology 17, 752-762.

Kniowski, A. \& Pietsch, R. (2015) Plethodon hubrichti (Peaks of Otter Salamander) Behavior. Herpetological Review 46, 71-71.

Kniowski, A. \& Reichenbach, N. (2009) The ecology of the Peaks of Otter salamander (Plethodon hubrichti) in sympatry with the eastern red-backed salamander (Plethodon cinereus). Herpetological Conservation and Biology 4, 285-294.

Kolozsvary, M.B. \& Swihart, R.K. (1999) Habitat fragmentation and the distribution of amphibians: patch and landscape correlates in farmland. Canadian Journal of Zoology 77, 1288-1299.

Kraemer, A.C. \& Adams, D.C. (2014) Predator Perception of Batesian Mimicry and Conspicuousness in a Salamander. Evolution 68, 1197-1206.

Kraemer, A.C., Kissner, J. \& Adams, D.C. (2012) Morphological Color-Change in the Red-Backed Salamander (Plethodon cinereus) While Kept in Captivity. Copeia 2012, 748-755.

Kraemer, A.C., Serb, J.M. \& Adams, D.C. (2015a) Batesian mimics influence the evolution of conspicuousness in an aposematic salamander. Journal of Evolutionary Biology 28, 1016-1023.

Kraemer, A.C., Serb, J.M. \& Adams, D.C. (2015b) Model toxin level does not directly influence the evolution of mimicry in the salamander Plethodon cinereus. Evolutionary Ecology 29, 511-523. 
Kraemer, A.C., Serb, J.M. \& Adams, D.C. (2016) Both novelty and conspicuousness influence selection by mammalian predators on the colour pattern of Plethodon cinereus (Urodela: Plethodontidae). Biological Journal of the Linnean Society 118, 889-900.

Kress, W.J., Erickson, D.L., Jones, F.A., Swenson, N.G., Perez, R., Sanjur, O. \& Bermingham, E. (2009) Plant DNA barcodes and a community phylogeny of a tropical forest dynamics plot in Panama. Proceedings of the National Academy of Sciences 106, 18621-18626.

Kroschel, W.A., Sutton, W.B., McClure, C.J.W. \& Pauley, T.K. (2014) Decline of the Cheat Mountain Salamander over a 32-Year Period and the Potential Influence of Competition from a Sympatric Species. Journal of Herpetology 48, 415-422.

Kunkel, C.L., Anthony, C.D., Hickerson, C.-A.M. \& Feldhoff, R.C. (2019) Species Variation in a Pheromone Complex is Maintained at the Population Level in the Eastern Red-Backed Salamander. Journal of Herpetology 53, 173-178.

Lancaster, D.L. \& Wise, S.E. (1996) Differential Response by the Ringneck Snake, Diadophis punctatus, to Odors of Tail-Autotomizing Prey. Herpetologica 52, 98-108.

Landres, P.B., Verner, J. \& Thomas, J.W. (1988) Ecological Uses of Vertebrate Indicator Species: A Critique. Conservation Biology 2, 316-328.

Lannoo, M. (ed) (2005) Amphibian Declines: The Conservation Status of United States Species First edition. University of California Press, Berkeley.

Lauer, A., Simon, M.A., Banning, J.L., André, E., Duncan, K. \& Harris, R.N. (2007) Common Cutaneous Bacteria from the Eastern Red-Backed Salamander Can Inhibit Pathogenic Fungi. Copeia 2007, 630-640.

Lauer, A., Simon, M.A., Banning, J.L., Lam, B.A. \& Harris, R.N. (2008) Diversity of cutaneous bacteria with antifungal activity isolated from female four-toed salamanders. The ISME Journal 2, 145-157.

Leclair, M.H., Levasseur, M. \& Leclair, R. (2008) Activity and Reproductive Cycles in Northern Populations of the Red-backed Salamander, Plethodon cinereus. Journal of Herpetology 42, 31-38. Society for the Study of Amphibians and Reptiles.

Leclair, M.H., Levasseur, M. \& Leclair, R., Jr (2006) Life-history traits of Plethodon cinereus in the northern parts of its range: variations in population structure, age, and growth. Herpetologica 62, 265-282.

LeGros, D.L. (2018) The use of an anthropogenic structure by Eastern Red-backed Salamander (Plethodon cinereus). The Canadian Field-Naturalist 132, 58-60.

Lehtinen, R.M., Steratore, A.F., Eyre, M.M., Cassagnol, E.S., Stern, M.L. \& Edgington, H.A. (2016) Identification of Widespread Hybridization between Two Terrestrial Salamanders Using Morphology, Coloration, and Molecular Markers. Copeia 104, 132-139.

Lemen, C.A. \& Freeman, P.W. (1985) Tracking Mammals with Fluorescent Pigments: A New Technique. Journal of Mammalogy 66, 134-136.

Leonelli, S. \& Ankeny, R.A. (2013) What makes a model organism? Endeavour 37, 209-212.

Leroux, S.J., Albert, C.H., Lafuite, A.-S., Rayfield, B., Wang, S. \& Gravel, D. (2017) Structural uncertainty in models projecting the consequences of habitat loss and fragmentation on biodiversity. Ecography 40, $36-47$.

Liebgold, E.B. (2014) The Influence of Social Environment: Behavior of Unrelated Adults Affects Future Juvenile Behaviors. Ethology 120, 388-399.

Liebgold, E.B. (2019) A tale of two tails: a rare occurrence of tail bifurcation in the red-backed salamander (Plethodon cinereus). Herpetological Bulletin 148, 35-36. 
Liebgold, E.B., Brodie, E.D. \& Cabe, P.R. (2011) Female philopatry and male-biased dispersal in a directdeveloping salamander, Plethodon cinereus. Molecular Ecology 20, 249-257.

Liebgold, E.B. \& Cabe, P.R. (2008) Familiarity with adults, but not relatedness, affects the growth of juvenile red-backed salamanders (Plethodon cinereus). Behavioral Ecology and Sociobiology 63, 277.

Liebgold, E.B. \& Dibble, C.J. (2011) Better the devil you know: familiarity affects foraging activity of red-backed salamanders, Plethodon cinereus. Animal Behaviour 82, 1059-1066.

Lindenmayer, D., Barton, P. \& Pierson, J. (2015) Indicators and Surrogates of Biodiversity and Environmental Change. Csiro Publishing.

Lotter, F. (1978) Reproductive Ecology of the Salamander Plethodon cinereus (Amphibia, Urodela, Plethodontidae) in Connecticut. Journal of Herpetology 12, 231-236.

Lotter, F. \& Scott, N.J. (1977) Correlation between Climate and Distribution of the Color Morphs of the Salamander Plethodon cinereus. Copeia 1977, 681-690.

Loudon, A.H., Holland, J.A., Umile, T.P., Burzynski, E.A., Minbiole, K.P.C. \& Harris, R.N. (2014a) Interactions between amphibians' symbiotic bacteria cause the production of emergent anti-fungal metabolites. Frontiers in Microbiology 5, 441.

Loudon, A.H., Venkataraman, A., Van Treuren, W., Woodhams, D.C., Parfrey, L.W., McKenzie, V.J., Knight, R., Schmidt, T.M. \& Harris, R.N. (2016) Vertebrate Hosts as Islands: Dynamics of Selection, Immigration, Loss, Persistence, and Potential Function of Bacteria on Salamander Skin. Frontiers in Microbiology 7,333 .

Loudon, A.H., Woodhams, D.C., Parfrey, L.W., Archer, H., Knight, R., McKenzie, V. \& Harris, R.N. (2014b) Microbial community dynamics and effect of environmental microbial reservoirs on red-backed salamanders (Plethodon cinereus). The ISME Journal 8, 830-840.

Luymes, N. \& Chow-Fraser, P. (2019) Optimizations for time and effort in long-term monitoring: a case study using a multidecadal terrestrial salamander monitoring program. Environmental Monitoring and Assessment 191, 597.

Lynn, W.G. \& Dent, J.N. (1941) Notes on the breeding habits of Plethodon cinereus. Copeia 1941, 113-114.

MacNeil, J.E. \& Williams, R.N. (2013) Effectiveness of two artificial cover objects in sampling terrestrial salamanders. Herpetological Conservation and Biology 8, 552-560.

MacNeil, J.E. \& Williams, R.N. (2014) Effects of Timber Harvests and Silvicultural Edges on Terrestrial Salamanders. PLOS ONE 9, e114683.

Madison, D.M., Sullivan, A.M., Maerz, J.C., McDarby, J.H. \& Rohr, J.R. (2002) A complex, cross-taxon, chemical releaser of antipredator behavior in amphibians. Journal of Chemical Ecology 28, 2271-2282.

Madison, D.M., Wareing, K. \& Maerz, J.C. (1999) Oviposition behavior in the red-backed salamander (Plethodon cinereus): Implications of suspending a clutch. Herpetological Review 30, 209-210.

Maerz, J., Myers, E. \& Adams, D. (2006) Trophic polymorphism in a terrestrial salamander. Evolutionary Ecology Research 8, 23-35.

Maerz, J.C. \& Karuzas, J.M. (2003) Plethodon cinereus (eastern red-backed salamander). Cannibalism. Herpetological Review 34, 354-354.

Maerz, J.C., Karuzas, J.M., Madison, D.M. \& Blossey, B. (2005) Introduced invertebrates are important prey for a generalist predator. Diversity and Distributions 11, 83-90.

Maerz, J.C. \& Madison, D.M. (2000) Environmental variation and territorial behavior in a terrestrial salamander. In Biology of Plethodontid Salamanders (eds R.C. Bruce, R.G. Jaeger \& L.D. Houck), pp. 395-406. 
Springer Science+.

Maerz, J.C., Panebianco, N.L. \& Madison, D.M. (2001) Effects of Predator Chemical Cues and Behavioral Biorhythms on Foraging, Activity of Terrestrial Salamanders. Journal of Chemical Ecology 27, 1333-1344.

Mahoney, K.R., Russell, K.R., Ford, W.M., Rodrigue, J.L., Riddle, J.D., Schuler, T.M. \& Adams, M.B. (2016) Woodland salamander responses to a shelterwood harvest-prescribed burn silvicultural treatment within Appalachian mixed-oak forests. Forest Ecology and Management 359, 277-285.

Marsh, D.M. (2007) Edge Effects of Gated and Ungated Roads on Terrestrial Salamanders. The Journal of Wildlife Management 71, 389-394.

Marsh, D.M. \& Beckman, N.G. (2004) Effects of Forest Roads on the Abundance and Activity of Terrestrial Salamanders. Ecological Applications 14, 1882-1891.

Marsh, D.M. \& Goicochea, M.A. (2003) Monitoring Terrestrial Salamanders: Biases Caused by Intense Sampling and Choice of Cover Objects. Journal of Herpetology 37, 460-466.

Marsh, D.M., Milam, G.S., Gorham, N.P. \& Beckman, N.G. (2005) Forest Roads as Partial Barriers to Terrestrial Salamander Movement. Conservation Biology 19, 2004-2008.

Marsh, D.M., Page, R.B., Hanlon, T.J., Bareke, H., Corritone, R., Jetter, N., Beckman, N.G., Gardner, K., Seifert, D.E. \& Cabe, P.R. (2007) Ecological and genetic evidence that low-order streams inhibit dispersal by red-backed salamanders (Plethodon cinereus). Canadian Journal of Zoology 85, 319-327.

Marsh, D.M., Page, R.B., Hanlon, T.J., Corritone, R., Little, E.C., Seifert, D.E. \& Cabe, P.R. (2008) Effects of roads on patterns of genetic differentiation in red-backed salamanders, Plethodon cinereus. Conservation Genetics 9, 603-613.

Marsh, D.M., Thakur, K.A., Bulka, K.C. \& Clarke, L.B. (2004) Dispersal and Colonization Through Open Fields by a Terrestrial, Woodland Salamander. Ecology 85, 3396-3405.

Marsh, D.M., Townes, F.W., Cotter, K.M., Farroni, K., McCreary, K.L., Petry, R.L. \& Tilghman, J.M. (2019) Thermal Preference and Species Range in Mountaintop Salamanders and Their Widespread Competitors. Journal of Herpetology 53, 96-103.

Martin, A.M. \& Hantak, M.M. (2011) Plethodon cinereus (Eastern Red-backed Salamander). Tenacity and Homing. Herpetological Review 42, 581-582.

Mathis, A. (1991) Territories of male and female terrestrial salamanders: costs, benefits, and intersexual spatial associations. Oecologia 86, 433-440.

Mathis, A. \& Lancaster, D. (1998) Response of terrestrial salamanders to chemical stimuli from distressed conspecifics. Amphibia-Reptilia 19, 330-335.

McCarthy, T., Masson, P., Thieme, A., Leimgruber, P. \& Gratwicke, B. (2017) The relationship between climate and adult body size in redback salamanders (Plethodon cinereus). Geo: Geography and Environment 4, e00031.

McGhee, J.D. (2013) Preliminary estimation of capture rates for red-backed salamanders at the RandolphMacon College Environmental Field Station, Doswell, Virginia. Catesbeiana 33, 62-66.

McGhee, J.D. \& Killian, M.D. (2012) Exploratory Modeling Indicates Red-Backed Salamander Detections are Sensitive to Soil pH at C. F. Phelps Wildlife Management Area, Virginia. Virginia Journal of Science 63, 137-144.

McKenny, H.C., Keeton, W.S. \& Donovan, T.M. (2006) Effects of structural complexity enhancement on eastern red-backed salamander (Plethodon cinereus) populations in northern hardwood forests. Forest Ecology and Management 230, 186-196. 
Meche, G. \& Jaeger, R.G. (2002) Associations of Male Red-Backed Salamanders with Tail-Intact versus Tail-Autotomized Females during the Courtship Season. Journal of Herpetology 36, 532-535. Society for the Study of Amphibians and Reptiles.

Mendyk, R.W., Augustine, L. \& Montague, G. (2010) Plethodon cinereus (Red-backed Salamander). Leucism. Herpetological Review 41, 189-190.

Meshaka, W.E., Lindsay, C.G., Mack, A.L. \& McCallum, M.L. (2019) A Survey of Terrestrially Active Salamanders from Two Different Woodlands at the Powdermill Nature Reserve, Pennsylvania. Northeastern Naturalist 26, 629-640.

Meshaka, W.E. \& Wright, D. (2017) Selected life history traits of the eastern red-backed salamander, Plethodon cinereus (Green), at a suburban residence in south central Pennsylvania. Journal of the Pennsylvania Academy of Science 91, 95-104.

Messere, M. \& Ducey, P.K. (1998) Forest floor distribution of northern redback salamanders, Plethodon cinereus, in relation to canopy gaps: first year following selective logging. Forest Ecology and Management 107, 319-324.

Mezebish, T.D., Blackman, A. \& Novarro, A.J. (2018) Salamander climbing behavior varies among species and is correlated with community composition. Behavioral Ecology 29, 686-692.

Milanovich, J.R. \& Maerz, J.C. (2012) Assessing the use of non-lethal tail clips for measuring stable isotopes of plethodontid salamanders. Herpetological Conservation and Biology 7, 67-74.

Milanovich, J.R. \& Maerz, J.C. (2013) Realistic Fasting Does Not Affect Stable Isotope Levels of a Metabolically Efficient Salamander. Journal of Herpetology 47, 544-548.

Mimubase.org (2021) Mimubase. http://mimubase.org/ [accessed 28 July 2021].

Mitchell, J.C. (2000) Observations on amphibians and reptiles in burned and unburned forests on the upper Coastal Plain of Virginia. Virginia Journal of Science 51, 199-203.

Mitchell, J.C. (2015) Amphibian and Small Mammal Assemblages in a Northern Virginia Forest Before and After Defoliation by Gypsy Moths (Lymantria dispar). Virginia Journal of Science 66, 403-411.

Mitchell, J.C., Rinehart, S.C., Pagels, J.F., Buhlmann, K.A. \& Pague, C.A. (1997) Factors influencing amphibian and small mammal assemblages in central Appalachian forests. Forest Ecology and Management 96, 65-76.

Monti, L., Hunter, M. \& Witham, J. (2000) An Evaluation of the Artificial Cover Object (ACO) Method for Monitoring Populations of the Redback Salamander Plethodon cinereus. Journal of Herpetology 34, 624-629.

Moore, A.L., Williams, C.E., Martin, T.H. \& Moriarity, W.J. (2001) Influence of Season, Geomorphic Surface and Cover Item on Capture, Size and Weight of Desmognathus ochrophaeus and Plethodon cinereus in Allegheny Plateau Riparian Forests. The American Midland Naturalist 145, 39-45.

Moore, J.-D. (2005) Use of Native Dominant Wood as a New Coverboard Type for Monitoring Eastern Red-backed Salamanders. Herpetological Review 36, 268-271.

Moore, J.-D. (2009) Comparison of a Population of Eastern Red-backed Salamanders, Plethodon cinereus, under Native Dominant Wood Coverboards and Natural Cover Objects. The Canadian Field-Naturalist 123, $210-214$.

Moore, J.-D. (2014) Short-term effect of forest liming on eastern red-backed salamander (Plethodon cinereus). Forest Ecology and Management 318, 270-273.

Moore, J.-D. \& Ouellet, M. (2014) A review of colour phenotypes of the Eastern Red-backed Salamander, Plethodon cinereus, in North America. The Canadian Field-Naturalist 128, 250-259. 
Moore, J.-D. \& Ouellet, M. (2015) Questioning the use of an amphibian colour morph as an indicator of climate change. Global Change Biology 21, 566-571.

Moore, J.-D. \& Wyman, R.L. (2010) Eastern Red-backed Salamanders (Plethodon cinereus) in a Highly Acid Forest Soil. The American Midland Naturalist 163, 95-105. University of Notre Dame.

Moreno, G. (1989) Behavioral and Physiological Differentiation between the Color Morphs of the Salamander, Plethodon cinereus. Journal of Herpetology 23, 335-341.

Mossman, A., Lambert, M.R., Ashton, M.S., Wikle, J. \& Duguid, M.C. (2019) Two salamander species respond differently to timber harvests in a managed New England forest. PeerJ 7, e7604.

Muletz, C.R., Myers, J.M., Domangue, R.J., Herrick, J.B. \& Harris, R.N. (2012) Soil bioaugmentation with amphibian cutaneous bacteria protects amphibian hosts from infection by Batrachochytrium dendrobatidis. Biological Conservation 152, 119-126.

Muletz-Wolz, C.R., DiRenzo, G.V., Yarwood, S.A., Campbell Grant, E.H., Fleischer, R.C. \& Lips, K.R. (2017) Antifungal Bacteria on Woodland Salamander Skin Exhibit High Taxonomic Diversity and Geographic Variability. Applied and Environmental Microbiology 83, e00186-17.

Muletz-Wolz, C.R., Fleischer, R.C. \& Lips, K.R. (2019) Fungal disease and temperature alter skin microbiome structure in an experimental salamander system. Molecular Ecology 28, 2917-2931.

Muletz-Wolz, C.R., Yarwood, S.A., Grant, E.H.C., Fleischer, R.C. \& Lips, K.R. (2018) Effects of host species and environment on the skin microbiome of Plethodontid salamanders. Journal of Animal Ecology 87, 341353.

Müller, B. \& Grossniklaus, U. (2010) Model organisms - A historical perspective. Journal of Proteomics 73, 2054-2063.

Muñoz, D.J. (2018) Caudata: Plethodon cinereus (Eastern red-backed salamander) Fluorescence. Herpetological Review 49, 512-513.

Muñoz, D.J., Hesed, K.M., Grant, E.H.C. \& Miller, D.A.W. (2016a) Evaluating within-population variability in behavior and demography for the adaptive potential of a dispersal-limited species to climate change. Ecology and Evolution 6, 8740-8755.

Muñoz, D.J., Miller, D.A.W., Sutherland, C. \& Grant, E.H.C. (2016b) Using Spatial Capture-Recapture to Elucidate Population Processes and Space-Use in Herpetological Studies. Journal of Herpetology 50, 570-581.

Murray, D.L. \& Jenkins, C.L. (1999) Perceived predation risk as a function of predator dietary cues in terrestrial salamanders. Animal Behaviour 57, 33-39.

Muzzall, P.M., Peebles, C.R. \& Burton, T.M. (1997) Endoparasites of Plethodontid Salamanders from Paradise Brook, New Hampshire. The Journal of Parasitology 83, 1193-1195.

Myers, E.M. \& Adams, D.C. (2008) Morphology is Decoupled from Interspecific Competition in Plethodon Salamanders in the Shenandoah Mountains, USA. Herpetologica 64, 281-289.

Nagel, J.W. (1977) Life History of the Red-Backed Salamander, Plethodon cinereus, in Northeastern Tennessee. Herpetologica 33, 13-18.

National Science Foundation (2021) Long Term Research in Environmental Biology | NSF - National Science Foundation. https://www.nsf.gov/funding/pgm_summ.jsp?pims_id=13544 [accessed 28 July 2021].

Ng, M.Y. \& Wilbur, H.M. (1995) The Cost of Brooding in Plethodon cinereus. Herpetologica 51, 1-8.

Noël, S. \& Lapointe, F.-J. (2010) Urban conservation genetics: Study of a terrestrial salamander in the city. Biological Conservation 143, 2823-2831. 
Noël, S., Ouellet, M., Galois, P. \& Lapointe, F.-J. (2007) Impact of urban fragmentation on the genetic structure of the eastern red-backed salamander. Conservation Genetics 8, 599-606.

Novarro, A.J., Gabor, C.R., Goff, C.B., Mezebish, T.D., Thompson, L.M. \& Grayson, K.L. (2018) Physiological responses to elevated temperature across the geographic range of a terrestrial salamander. Journal of Experimental Biology 221, jeb178236.

Ochs, A. \& Siddig, A.A.H. (2017) Response of Red-Backed Salamanders (Plethodon cinereus) to Changes in Hemlock Forest Soil Driven by Invasive Hemlock Woolly Adelgid (Adelges tsugae). Environments 4, 8.

Orlofske, S.A., Grayson, K.L. \& Hopkins, W.A. (2009) The Effects of Fluorescent Tracking Powder on Oxygen Consumption in Salamanders Using Either Cutaneous or Bimodal Respiration. Copeia 2009, 623-627.

Orr, J.M. \& Ernst, C.H. (2005) Plethodon cinereus (Eastern red-backed salamander). Early nesting. Herpetological Review 36, 296-296.

Otaibi, B.W., Johnson, Q.K. \& Cosentino, B.J. (2017) Postautotomy tail movement differs between colour morphs of the red-backed salamander (Plethodon cinereus). Amphibia-Reptilia 38, 395-399.

Otto, C.R.V., Bailey, L.L. \& Roloff, G.J. (2013) Improving species occupancy estimation when sampling violates the closure assumption. Ecography 36, 1299-1309.

Otto, C.R.V. \& Roloff, G.J. (2011) Comparing Cover Object and Leaf Litter Surveys for Detecting RedBacked Salamanders, Plethodon cinereus. Journal of Herpetology 45, 256-260. Society for the Study of Amphibians and Reptiles.

Otto, C.R.V., Roloff, G.J. \& Thames, R.E. (2014) Comparing Population Patterns to Processes: Abundance and Survival of a Forest Salamander following Habitat Degradation. PLOS ONE 9, e93859.

Ousterhout, B.H. \& Liebgold, E.B. (2010) Dispersal Versus Site Tenacity of Adult and Juvenile Red-Backed Salamanders (Plethodon cinereus). Herpetologica 66, 269-275.

Paluh, D.J., Eddy, C., Ivanov, K., Hickerson, C.-A.M. \& Anthony, C.D. (2015) Selective Foraging on Ants by a Terrestrial Polymorphic Salamander. The American Midland Naturalist 174, 265-277.

Pasachnik, S. \& Ruthig, G.R. (2004) Versatility of Habitat Use in Three Sympatric Species of Plethodontid Salamanders. Journal of Herpetology 38, 434-437.

Passos, L.F., Garcia, G. \& Young, R.J. (2018) Comparing the bacterial communities of wild and captive golden mantella frogs: Implications for amphibian conservation. PLOS ONE 13, e0205652.

Patrick, D.A., Hunter, M.L. \& Calhoun, A.J.K. (2006) Effects of experimental forestry treatments on a Maine amphibian community. Forest Ecology and Management 234, 323-332.

Peele, J., Nix, C., Ruhl, P., Chapman, R., Zollner, P. \& Saunders, M.R. (2017) Effects of Woody Biomass Harvests on a Population of Plethodontid Salamanders in Southeast Indiana. The American Midland Naturalist 178, 132-143.

Pelini, S.L., Maran, A.M., Chen, A.R., Kaseman, J. \& Crowther, T.W. (2015) Higher Trophic Levels Overwhelm Climate Change Impacts on Terrestrial Ecosystem Functioning. PLOS ONE 10, e0136344.

Perkins, D.W. \& Hunter, M.L. (2006) Effects of Riparian Timber Management on Amphibians in Maine. The Journal of Wildlife Management 70, 657-670.

Peterson, M.G., Gillette, J.R., Franks, R. \& Jaeger, R.G. (2000) Alternative life styles in a terrestrial salamander. In Biology of Plethodontid Salamanders (eds R.C. Bruce, R.G. Jaeger \& L.D. Houck), pp. 417-430. Springer Science + .

Petranka, J. (1998) Salamanders of the United States and Canada 1st edition. Smithsonian Books, Washington. 
Petruzzi, E.E., Niewiarowski, P.H. \& Moore, F.B.-G. (2006) The role of thermal niche selection in maintenance of a colour polymorphism in redback salamanders (Plethodon cinereus). Frontiers in Zoology 3, 10.

Piersol, W.H. (1910) The habits and larval state of Plethodon cinereus erythrionotus. Transactions of the Royal Canadian Institute 8, 469-492.

Placyk, J.S. \& Graves, B.M. (2001a) Foraging Behavior of the Red-Backed Salamander (Plethodon cinereus) under Various Lighting Conditions. Journal of Herpetology 35, 521-524.

Placyk, J.S. \& Graves, B.M. (2001b) Plethodon cinereus (Red-backed salamander). Site attachment. Herpetological Review 32, 246-246.

Placyk, J.S. \& Graves, B.M. (2002) Prey Detection by Vomeronasal Chemoreception in a Plethodontid Salamander. Journal of Chemical Ecology 28, 1017-1036.

Placyk, J.S., Seider, M.J. \& Gillingham, J.C. (2002) New herpetological records for High and Hog Islands of the Beaver Archipelago, Charlevoix County, Michigan. Herpetological Review 33, 230-230.

Placyk, J.S., Torretti, L. \& Graves, B.M. (2000) Plethodon cinereus (Red-backed Salamander). Intraspecific aggregation. Herpetological Review 31, 167-167.

Price, J.E. \& Secki Shields, J.A. (2002) Size-dependent interactions between two terrestrial amphibians, Plethodon cinereus and Plethodon glutinosus. Herpetologica 58, 141-155.

Quinn, V.S. \& Graves, B.M. (1999a) Space Use in Response to Conspecifics by the Red-backed Salamander (Plethodon cinereus, Plethodontidae, Caudata). Ethology 105, 993-1002.

Quinn, V.S. \& Graves, B.M. (1999b) A technique for sexing red-backed salamanders (Plethodon cinereus). Herpetological Review 30, 32. Society for the Study of Amphibians and Reptiles, St. Louis, United States.

Radomski, T., Hantak, M.M., Brown, A.D. \& Kuchta, S.R. (2020) Multilocus Phylogeography of Eastern RedBacked Salamanders (Plethodon cinereus): Cryptic Appalachian Diversity and Postglacial Range Expansion. Herpetologica 76, 61-73.

Raimondo, S., Pauley, T.K. \& Butler, L. (2003) Potential impacts of Bacillus thuringiensis var. kurstaki on five salamander species in West Virginia. Northeastern Naturalist 10, 25-38.

Ransom, T.S. (2012) Comparison of direct, indirect, and ecosystem engineering effects of an earthworm on the red-backed salamander. Ecology 93, 2198-2207.

Ransom, T.S. (2017) Local distribution of native and invasive earthworms and effects on a native salamander. Population Ecology 59, 189-204.

Ransom, T.S. \& Jaeger, R.G. (2006) An Assemblage of Salamanders in the Southern Appalachian Mountains Revisited: Competitive and Predatory Behavior? Behaviour 143, 1357-1382.

Ransom, T.S. \& Jaeger, R.G. (2008) Intergeneric salamander interactions across an ecotone. Herpetologica 64, 20-31.

Reams, R.D., Searcy, R., Wyatt, J.E. \& Gehrmann, W.H. (2008) Habitat utilization by reptiles and amphibians at an urban state park in Indiana. Bulletin of the Chicago Herpetological Society 43, 17-20.

Reiter, M.K., Anthony, C.D. \& Hickerson, C.-A.M. (2014) Territorial Behavior and Ecological Divergence in a Polymorphic Salamander. Copeia 2014, 481-488.

Renaldo, K., Murch, C., Riley, J.-N., Helleman, B., Smith, G. \& Rettig, J. (2011) Substrate preference of eastern red-backed salamanders, Plethodon cinereus: A comparison of deciduous and coniferous substrates. Amphibia-Reptilia 32, 266-269. 
Richmond, L.S. \& Trombulak, S.C. (2009) Distribution of Red-Backed Salamander (Plethodon cinereus) with Respect to Cover-Object Characteristics in the Green Mountains of Vermont. Northeastern Naturalist 16, 13-26.

Riedel, B.L., Russell, K.R. \& Ford, W.M. (2012) Physical Condition, Sex, and Age-Class of Eastern RedBacked Salamanders (Plethodon cinereus) in Forested and Open Habitats of West Virginia, USA. International Journal of Zoology 2012, e623730.

Riedel, B.L., Russell, K.R., Ford, W.M., O'Neill, K.P. \& Godwin, H.W. (2008) Habitat relationships of eastern red-backed salamanders (Plethodon cinereus) in Appalachian agroforestry and grazing systems. Agriculture, Ecosystems $\&$ Environment 124, 229-236.

Roberts, A.M. \& Liebgold, E.B. (2008) The effects of perceived mortality risk on habitat selection in a terrestrial salamander. Behavioral Ecology 19, 621-626.

Roble, S.M. (1999) Plethodon cinereus (red-backed salamander).Catesbeiana 19, 33-33.

Rodewald, A.D. \& Yahner, R.H. (1999) Effects of forest management and landscape composition on woodland salamanders. Northeast Wildlife 54, 45-54.

Rollinson, N. \& Hackett, D. (2015) Experimental evaluation of agonistic behaviour, chemical communication, spacing, and intersexual associations of the Eastern Red-backed Salamander (Plethodon cinereus) near its northern range limit. Canadian Journal of Zoology 93, 773-781.

Rooney, T.P., Antolik, C. \& Moran, M.D. (2000) The impact of salamander predation on Collembola abundance. Proceedings of the Entomological Society of Washington 102, 308-312.

Russell, J.J., Theriot, J.A., Sood, P., Marshall, W.F., Landweber, L.F., Fritz-Laylin, L., Polka, J.K., Oliferenko, S., Gerbich, T., Gladfelter, A., Umen, J., Bezanilla, M., Lancaster, M.A., He, S., Gibson, M.C., et al. (2017) Non-model model organisms. BMC Biology 15, 55.

Russell, R.W. (2011) Plethodon cinereus (Eastern Red-backed Salamander). Wildfire Survival. Herpetological Review 42, 359-262.

Sawyers, M.S. \& Novick, C.M.J. (2011) Plethodon cinereus (Eastern Red-backed Salamander). Color variation. Herpetological Review 42, 580-581.

Sayler, A. (1966) The Reproductive Ecology of the Red-Backed Salamander, Plethodon cinereus, in Maryland. Copeia 1966, 183-193.

Schieltz, J.M., Haywood, L.M.B. \& Marsh, D.M. (2010) Effects of Cover Object Spacing on the Socioecology of the Red-Backed Salamander, Plethodon cinereus. Herpetologica 66, 276-282.

Schluter, D. (2000) The Ecology of Adaptive Radiation 1st edition. Oxford University Press, Oxford.

Seburn, D.C. \& Mallon, E. (2017) Has the Eastern Red-backed Salamander (Plethodon cinereus) Declined in Ontario? The Canadian Field-Naturalist 131, 115-119.

Semlitsch, R.D., O’Donnell, K.M. \& Thompson, F.R. (2014) Abundance, biomass production, nutrient content, and the possible role of terrestrial salamanders in Missouri Ozark forest ecosystems. Canadian Journal of Zoology 92, 997-1004.

Sever, D.M. (1997) Sperm storage in the spermatheca of the red-back salamander, Plethodon cinereus (Amphibia: Plethodontidae).Journal of Morphology 234, 131-146.

Sever, D.M. (2017) Ultrastructure of the mental gland of the Red-Backed Salamander, Plethodon cinereus (Amphibia: Plethodontidae). Acta Zoologica 98, 154-162.

Sever, D.M. \& Siegel, D.S. (2006) Sperm aggregations in the spermatheca of the red back salamander (Plethodon cinereus). Acta Zoologica 87, 331-340. 
Sever, D.M. \& Siegel, D.S. (2015) Histology and ultrastructure of the caudal courtship glands of the redbacked salamander, Plethodon cinereus (Amphibia: Plethodontidae). Journal of Morphology 276, 319-330.

Sharp, C.C., Steckler, S.E., Lockhart, O.M. \& Walton, B.M. (2005) Plethodon cinereus (Eastern red-backed salamander). Predation. Herpetological Review 36, 296-297.

Shortlidge, E.E., Bangera, G. \& Brownell, S.E. (2017) Each to Their Own CURE: Faculty Who Teach Course-Based Undergraduate Research Experiences Report Why You Too Should Teach a CURE+. Journal of Microbiology \&3 Biology Education 18, 10.

Siddig, A.A., Ellison, A.M. \& Jackson, S. (2015) Calibrating abundance indices with population size estimators of red back salamanders (Plethodon cinereus) in a New England forest. PeerJ 3, e952.

Siddig, A.A.H., Ochs, A. \& Ellison, A.M. (2019) Do Terrestrial Salamanders Indicate Ecosystem Changes in New England Forests? Forests 10, 154.

Silva, M., Hartling, L.A., Field, S.A. \& Teather, K. (2003) The effects of habitat fragmentation on amphibian species richness of Prince Edward Island. Canadian Journal of Zoology 81, 563-573.

Simons, R.R., Felgenhauer, B.E. \& Thompson, T. (1999) Description of the postcloacal glands of Plethodon cinereus, the red-backed salamander, during bouts of scent marking. Journal of Morphology 242, 257-269.

Sites, J.W., Morando, M., Highton, R., Huber, F. \& Jung, R.E. (2004) Phylogenetic Relationships of the Endangered Shenandoah Salamander (Plethodon shenandoah) and Other Salamanders of the Plethodon cinereus Group (Caudata: Plethodontidae). Journal of Herpetology 38, 96-105.

Smith, G.R. (2018) Does Removal of Invasive Garlic Mustard Affect Eastern Red-backed Salamanders? Ecological Restoration 36, 113-115.

Smith, G.R., Johnson, T. \& Smith, W.O. (2015) Effects of colour morph and season on the dehydration and rehydration rates of Plethodon cinereus. Amphibia-Reptilia 36, 170-174.

SPARCnet.org (2021) The Salamander Population \& Adaptation Research Collaboration Network. http: //sparcnet.org/ [accessed 28 July 2021].

Staub, N.L., Stiller, A.B. \& Kiemnec-Tyburczy, K.M. (2020) A New Perspective on Female-to-Male Communication in Salamander Courtship. Integrative and Comparative Biology 60, 722-731.

Strojny, C.J. \& Hunter, M.L. (2010a) Log diameter influences detection of eastern red-backed salamanders (Plethodon cinereus) in harvest gaps but not in closed-canopy forest conditions. Herpetological Conservation and Biology 5, 80-85.

Strojny, C.J. \& Hunter, M.L. (2010b) Comparing relative abundance of amphibians in forest canopy gaps of natural origin vs. timber harvest origin. Animal Biodiversity and Conservation 33, 1.

Stuczka, A., Hickerson, C.-A. \& Anthony, C. (2016) Niche partitioning along the diet axis in a colour polymorphic population of Eastern Red-backed Salamanders, Plethodon cinereus. Amphibia-Reptilia 37, 283-290.

Sucre, E.B., Homyack, J.A., Fox, T.R. \& Haas, C.A. (2010) The influence of red-backed salamanders (Plethodon cinereus) on nutrient cycling in Appalachian hardwood forests. In: Stanturf, John A., ed. 2010. Proceedings of the 14 th biennial southern silvicultural research conference. Gen. Tech. Rep. SRS121. Asheville, NC: U.S. Department of Agriculture, Forest Service, Southern Research Station. 487-493. 121, 487-493.

Sugalski, M.T. \& Claussen, D.L. (1997) Preference for Soil Moisture, Soil pH, and Light Intensity by the Salamander, Plethodon cinereus. Journal of Herpetology 31, 245-250.

Sullivan, A., Rohr, J. \& Madison, D. (2003) Behavioural Responses by Red-backed Salamanders to Conspecific and Heterospecific Cues. Behaviour 140, 553-564. 
Sullivan, A.M. \& Jensen, G. (2013) A Study of the Sensitivity of Plethodon cinereus (Caudata: Plethodontidae) to Damage-Released Cues from Conspecifics. Journal of Herpetology 47, 585-589.

Sullivan, A.M., Madison, D.M. \& Rohr, J.R. (2004) Variation in the antipredator responses of three sympatric plethodontid salamanders to predator-diet cues. Herpetologica 60, 401-408.

Sullivan, A.M., Maerz, J.C. \& Madison, D.M. (2002) Anti-predator response of red-backed salamanders (Plethodon cinereus) to chemical cues from garter snakes (Thamnophis sirtalis): laboratory and field experiments. Behavioral Ecology and Sociobiology 51, 227-233.

Sullivan, A.M., Picard, A.L. \& Madison, D.M. (2005) To avoid or not to avoid? Factors influencing the discrimination of predator diet cues by a terrestrial salamander. Animal Behaviour 69, 1425-1433.

Sutherland, C., Munoz, D.J., Miller, D.A.W. \& Grant, E.H.C. (2016) Spatial Capture-Recapture: A Promising Method for Analyzing Data Collected Using Artificial Cover Objects. Herpetologica 72, 6-12.

Sutherland, W.J., Freckleton, R.P., Godfray, H.C.J., Beissinger, S.R., Benton, T., Cameron, D.D., Carmel, Y., Coomes, D.A., Coulson, T., Emmerson, M.C., Hails, R.S., Hays, G.C., Hodgson, D.J., Hutchings, M.J., Johnson, D., et al. (2013) Identification of 100 fundamental ecological questions. Journal of Ecology 101, $58-67$.

Swart, C.C. \& Adams, D.C. (2004) The role of muscle mass and tooth number in ecological character displacement between Plethodon cinereus and P. hoffmani (Caudata: Plethodontidae). Herpetologica 60, 408-413.

Takahashi, M.K. \& Pauley, T.K. (2010) Resource Allocation and Life History Traits of Plethodon cinereus at Different Elevations. The American Midland Naturalist 163, 87-94.

Telfer, A.C. \& Laberge, F. (2013) Responses of Eastern red-backed salamanders (Plethodon cinereus) to chemical cues of prey presented in soluble and volatile forms. Physiology \& Behavior 114-115, 6-13.

Test, F.H. (1952) Spread of the Black Phase of the Red-Backed Salamander in Michigan. Evolution 6, 197-203.

Tinbergen, N. (1963) On aims and methods of Ethology. Zeitschrift fur Tierpsychologie 20, 410-433.

Tornick, J.K. (2010) Factors Affecting Aggression during Nest Guarding in the Eastern Red-Backed Salamander (Plethodon cinereus). Herpetologica 66, 385-392.

Townsend, J.M. \& Driscoll, C.T. (2013) Red-backed salamander (Plethodon cinereus) as a bioindicator of mercury in terrestrial forests of the northeastern United States. Ecological Indicators 34, 168-171.

Travis, J. (2006) Is It What We Know or Who We Know? Choice of Organism and Robustness of Inference in Ecology and Evolutionary Biology. (American Society of Naturalists Presidential Address). The American Naturalist 167, 303-314.

Uller, C., Jaeger, R., Guidry, G. \& Martin, C. (2003) Salamanders (Plethodon cinereus) go for more: rudiments of number in an amphibian. Animal Cognition 6, 105-112.

Vanderwel, M.C., Caspersen, J.P., Malcolm, J.R., Papaik, M.J. \& Messier, C. (2011) Structural changes and potential vertebrate responses following simulated partial harvesting of boreal mixedwood stands. Forest Ecology and Management 261, 1362-1371.

Venesky, M.D. \& Anthony, C.D. (2007) Antipredator adaptations and predator avoidance by two color morphs of the eastern red-backed salamander, Plethodon cinereus. Herpetologica 63, 450-458.

Venesky, M.D., Hess, A., DeMarchi, J.A., Weil, A., Murone, J., Hickerson, C. -A. M. \& Anthony, C.D. (2015) Morph-specific differences in disease prevalence and pathogen-induced mortality in a terrestrial polymorphic salamander. Journal of Zoology 295, 279-285. 
Venier, L.A., Pearce, J.L., Wintle, B.A. \& Bekessy, S.A. (2007) Future forests and indicator-species population models. The Forestry Chronicle 83, 36-40.

VertNet (2021) VertNet-Home. http://www.vertnet.org/index.html [accessed 19 July 2021].

Wake, D. \& Lynch, J. (1976) The distribution, ecology, and evolutionary history of plethodontid salamanders in tropical America. Science Bulletin of the Natural History Museum of Los Angeles County 25, 1-65.

Wake, D.B. \& Hanken, J. (2004) Direct development in the lungless salamanders: what are the consequences for developmental biology, evolution and phylogenesis? International Journal of Developmental Biology 40, 859-869.

Waldick, R.C., Freedman, B. \& Wassersug, R.J. (1999) The consequences for amphibians of the conversion of natural, mixed-species forests to conifer plantations in southern New Brunswick. Canadian Field-Naturalist 113, 408-418.

Waldron, B.P., Kuchta, S.R., Hantak, M.M., Hickerson, C.-A.M. \& Anthony, C.D. (2019) Genetic Analysis of a Cryptic Contact Zone between Mitochondrial Clades of the Eastern Red-Backed Salamander, Plethodon cinereus. Journal of Herpetology 53, 144-153.

Wale, N. \& Duffy, M.A. (2021) The Use and Underuse of Model Systems in Infectious Disease Ecology and Evolutionary Biology. The American Naturalist 198, 69-92.

Walker, D.M., Lawrence, B.R., Esterline, D., Graham, S.P., Edelbrock, M.A. \& Wooten, J.A. (2014) A metagenomics-based approach to the top-down effect on the detritivore food web: a salamanders influence on fungal communities within a deciduous forest. Ecology and Evolution 4, 4106-4116.

Walker, D.M., Lawrence, B.R., Esterline, D., McAndrew, M., Edelbrock, J., Graham, S.P. \& Kelehear, C. (2015) A Novel Protocol for Washing Environmental Microbes from Amphibian Skin. Herpetological Review 46, 349-353.

Walker, D.M., Murray, C.M., Talbert, D., Tinker, P., Graham, S.P. \& Crowther, T.W. (2018) A salamander's top down effect on fungal communities in a detritivore ecosystem. FEMS Microbiology Ecology 94, fiy168.

Walton, B.M. (2005) Salamanders in forest-floor food webs: Environmental heterogeneity affects the strength of top-down effects. Pedobiologia 49, 381-393.

Walton, B.M. \& Steckler, S. (2005) Contrasting effects of salamanders on forest-floor macro- and mesofauna in laboratory microcosms. Pedobiologia 49, 51-60.

Walton, B.M., Tsatiris, D. \& Rivera-Sostre, M. (2006) Salamanders in forest-floor food webs: Invertebrate species composition influences top-down effects. Pedobiologia 50, 313-321.

Wells, K.D. (2007) The Ecology and Behavior of Amphibians. University of Chicago Press.

Welsh, H.H. \& Droege, S. (2001) A Case for Using Plethodontid Salamanders for Monitoring Biodiversity and Ecosystem Integrity of North American Forests. Conservation Biology 15, 558-569.

Welsh, H.H. \& Ollivier, L.M. (1998) Stream Amphibians as Indicators of Ecosystem Stress: a Case Study from California's Redwoods. Ecological Applications 8, 1118-1132.

Wiens, J.J. (2007) Global Patterns of Diversification and Species Richness in Amphibians. The American Naturalist 170, S86-S106.

Wiens, J.J., Engstrom, T.N. \& Chippindale, P.T. (2006) Rapid Diversification, Incomplete Isolation, and the "Speciation Clock" in North American Salamanders (genus Plethodon): Testing the Hybrid Swarm Hypothesis of Rapid Radiation. Evolution 60, 2585-2603. 
Wiggins, P.J., Smith, J.M., Harris, R.N. \& Minbiole, K.P.C. (2011) Gut of Red-backed Salamanders (Plethodon cinereus) May Serve as a Reservoir for an Antifungal Cutaneous Bacterium. Journal of Herpetology 45, 329-332.

Wilbur, H.M. (1980) Complex Life Cycles. Annual Review of Ecology and Systematics 11, 67-93.

Wilburn, D.B., Bowen, K.E., Feldhoff, P.W. \& Feldhoff, R.C. (2014) Proteomic Analyses of Courtship Pheromones in the Redback Salamander, Plethodon cinereus. Journal of Chemical Ecology 40, 928-939.

Wilk, A.J., Donlon, K.C. \& Peterman, W.E. (2020) Effects of habitat fragment size and isolation on the density and genetics of urban red-backed salamanders (Plethodon cinereus).Urban Ecosystems 23, 761-773.

Willey, L.L. \& Jones, M.T. (2010) Plethodon cinereus (Red-backed Salamander). Elevation. Herpetological Review 41, 190-190.

Williams, E.E., Highton, R. \& Cooper, D.M. (1968) Breakdown of Polymorphism of the Red-Backed Salamander on Long Island. Evolution 22, 76-86.

Wise, S.E. \& Jaeger, R.G. (1998) The influence of tail autotomy on agonistic behaviour in a territorial salamander. Animal Behaviour 55, 1707-1716.

Wood, P.B. \& Williams, J.M. (2013) Terrestrial salamander abundance on reclaimed mountaintop removal mines. Wildlife Society Bulletin 37, 815-823.

Woolbright, L.L. \& Martin, C.P. (2014) Seasonal Migration by Red-Backed Salamanders, Plethodon cinereus. Journal of Herpetology 48, 546-551.

Wunder, J.L., Lampazzi, N.M., Acre, K.D., Bent, N.J., Canter, S.A., Chapman, A.M., Davies, M.A., Kashan, D., Keiley, J.W., Macintyre, R.I., Milton, T.F., Weichler, K.L., Wilson, M.J. \& Takahashi, M.K. (2012) Promoting amphibian conservation through the college classroom: detection of Batrachochytrium dendrobatidis among local amphibians. Herpetological Conservation and Biology 7, 462-469.

Wyman, R.L. (1988) Soil Acidity and Moisture and the Distribution of Amphibians in Five Forests of Southcentral New York. Copeia 1988, 394-399.

Wyman, R.L. (1998) Experimental assessment of salamanders as predators of detrital food webs: effects on invertebrates, decomposition and the carbon cycle. Biodiversity \& Conservation 7, 641-650.

Yahner, R.H., Bramble, W.C. \& Byrnes, W.R. (2001a) Response of amphibian and reptile populations to vegetation maintenance of an electric transmission line right-of-way. Journal of Aboriculture 27, 215-221.

Yahner, R.H., Piergallini, N.H. \& Ross, B.D. (2001b) Survey of amphibians and reptiles in two types of managed forests in central Pennsylvania. Journal of the Pennsylvania Academy of Science 74, 48-51.

Young, G.I. \& Yahner, R.H. (2003) Distribution of, and microhabitat use by, woodland salamanders along forest-farmland edges. Canadian Field-Naturalist 117, 19-24.

Yu, Z., Dickstein, R., Magee, W.E. \& Spotila, J.R. (1998) Heat shock response in the salamanders Plethodon jordani and Plethodon cinereus. Journal of Thermal Biology 23, 259-265.

Yurewicz, K.L. \& Wilbur, H.M. (2004) Resource Availability and Costs of Reproduction in the Salamander Plethodon cinereus. Copeia 2004, 28-36.

Ziemba, J.L., Cameron, A.C., Peterson, K., Hickerson, C.-A.M. \& Anthony, C.D. (2015) Invasive Asian earthworms of the genus Amynthas alter microhabitat use by terrestrial salamanders. Canadian Journal of Zoology 93, 805-811.

Ziemba, J.L., Hickerson, C.-A.M. \& Anthony, C.D. (2016) Invasive Asian Earthworms Negatively Impact Keystone Terrestrial Salamanders. PLOS ONE 11, e0151591.

\section{Supporting Information}


Table S1.xlsx. List of number of source for each of 50 genera searched during genus-level search

Table S2.xlsx. List of number of source for each of 179 species searched during species-level search

Table S3.xlsx - Table S10.xlsx. List of sources for each of the top 8 most well-studied salamanders

Table S11.xlsx. Categorization \& summaries of post-Petranka literature on Plethodon cinereus

Table S12.xlsx. Geographic range size data for all salamander species available from the IUCN

Table S13.xlsx. Categorization of sources cited by Petranka

Table S14.xlsx. Number of post-Petranka articles by state/province for Plethodon cinereus

Supplemental Figures.docx. Document of four supplemental figures and their captions

Figure S1. Natural tail fluorescence in Plethodon cinereus from Massachusetts

Figure S2. High density coverboards sharing in Plethodon cinereus from Virginia

Figure S3. An example of a bifurcated tail in Plethodon cinereus from Pennsylvania

Figure S4. Map of SPARCnet participants across the range of Plethodon cinereus 